Supplementary Materials for

\title{
A Solution-Phase Approach to Functionalized Janus Dendrimers: Novel Soluble Supports for Organic Synthesis
}

\author{
Yu Feng, ${ }^{\text {a, b }}$ Yan-Mei He, ${ }^{\text {a Li-Wen Zhao, }}$, Yi-Yong Huang, ${ }^{\text {a, b }}$ Qing-Hua Fan ${ }^{\text {a,* }}$ \\ ${ }^{a}$ Beijing National Laboratory for Molecular Sciences, Center for Chemical Biology, Institute \\ of Chemistry, Chinese Academy of Sciences, Beijing 100080, China; \\ ${ }^{b}$ Graduate School of the Chinese Academy of Sciences, Beijing 100080, China.
}

fanqh@iccas.ac.cn

\section{Table of Contents}

1. General information ---

2. General procedure for the synthesis of Janus dendrimers --

3. Janus dendrimers as soluble supports for the Pd-catalyzed Suzuki coupling reactions

4. ${ }^{1} \mathrm{H}$ and ${ }^{13} \mathrm{C}$ NMR and MS spectra of Janus dendrimers ---

Figure S1. ${ }^{1} \mathrm{H}$ NMR spectra of dendrimer $\mathbf{G}_{3} \mathbf{C H}_{2}-\mathbf{O H}$

Figure S2. ${ }^{1} \mathrm{H}$ NMR, ${ }^{13} \mathrm{C}$ NMR and MS spectra of Janus dendrimer $\mathbf{G}_{\mathbf{3}} \mathbf{G}_{\mathbf{1}}-\mathbf{C O O C H} \mathbf{H}_{\mathbf{3}}--------------16$

Figure S3. ${ }^{1} \mathrm{H}$ NMR, ${ }^{13} \mathrm{C}$ NMR and MS spectra of Janus dendrimer $\mathbf{G}_{\mathbf{3}} \mathbf{G}_{\mathbf{1}}-\mathbf{C} \mathbf{H}_{\mathbf{2}} \mathbf{O H}$------------------17

Figure S4. ${ }^{1} \mathrm{H}$ NMR, ${ }^{13} \mathrm{C}$ NMR and MS spectra of Janus dendrimer $\mathbf{G}_{3} \mathbf{G}_{2}-\mathbf{C O O C H} \mathbf{H}_{3}-------------19$

Figure S5. ${ }^{1} \mathrm{H}$ NMR, ${ }^{13} \mathrm{C}$ NMR and MS spectra of Janus dendrimer $\mathbf{G}_{3} \mathbf{G}_{\mathbf{2}}-\mathbf{C H}_{\mathbf{2}} \mathbf{O H}-----------------20$

Figure S6. ${ }^{1} \mathrm{H}$ NMR, ${ }^{13} \mathrm{C}$ NMR and MS spectra of Janus dendrimer $\mathbf{G}_{3} \mathbf{G}_{3}-\mathbf{C O O C} \mathbf{H}_{3}--------------22$

Figure S7. ${ }^{1} \mathrm{H}$ NMR, ${ }^{13} \mathrm{C}$ NMR and MS spectra of Janus dendrimer $\mathbf{G}_{3} \mathbf{G}_{3}-\mathbf{C H}_{\mathbf{2}} \mathbf{O H}----------------23$

Figure S8. ${ }^{1} \mathrm{H}$ NMR, ${ }^{13} \mathrm{C}$ NMR and MS spectra of Janus dendrimer $\mathbf{G}_{3} \mathbf{G}^{\prime}{ }_{3}-\mathbf{C O O C H} \mathbf{H}^{-------------25}$

Figure S9. ${ }^{1} \mathrm{H}$ NMR, ${ }^{13} \mathrm{C}$ NMR and MS spectra of Janus dendrimer $\mathbf{G}_{3} \mathbf{G}^{\prime}{ }_{3}-\mathbf{C H}_{2} \mathbf{O H}---------------26$ 
5. ${ }^{1} \mathrm{H}$ and ${ }^{13} \mathrm{C}$ NMR and MS spectra of the dendritic Suzuki coupling products ----28-44

Figure S10. ${ }^{1} \mathrm{H}$ NMR, ${ }^{13} \mathrm{C}$ NMR and MS spectra of Janus dendrimer 3a------------------------------28

Figure S11. ${ }^{1} \mathrm{H}$ NMR, ${ }^{13} \mathrm{C}$ NMR and MS spectra of Janus dendrimer $\mathbf{3 b}$------------------------------29

Figure S12. ${ }^{1} \mathrm{H}$ NMR, ${ }^{13} \mathrm{C}$ NMR and MS spectra of Janus dendrimer 3c-----------------------------31

Figure S13. ${ }^{1} \mathrm{H}$ NMR, ${ }^{13} \mathrm{C}$ NMR and MS spectra of Janus dendrimer 3d------------------------------32

Figure S14. ${ }^{1} \mathrm{H}$ NMR, ${ }^{13} \mathrm{C}$ NMR and MS spectra of Janus dendrimer 4aa-----------------------------34

Figure S15. ${ }^{1} \mathrm{H}$ NMR, ${ }^{13} \mathrm{C}$ NMR and MS spectra of Janus dendrimer 4ba-----------------------------35

Figure S16. ${ }^{1} \mathrm{H}$ NMR, ${ }^{13} \mathrm{C}$ NMR and MS spectra of Janus dendrimer 4ca------------------------------37

Figure S17. ${ }^{1} \mathrm{H}$ NMR, ${ }^{13} \mathrm{C}$ NMR and MS spectra of Janus dendrimer 4da----------------------------38

Figure S18. ${ }^{1} \mathrm{H}$ NMR, ${ }^{13} \mathrm{C}$ NMR and MS spectra of Janus dendrimer 4cb-----------------------------40

Figure S19. ${ }^{1} \mathrm{H}$ NMR, ${ }^{13} \mathrm{C}$ NMR and MS spectra of Janus dendrimer 4cc---------------------------41

Figure S20. ${ }^{1} \mathrm{H}$ NMR, ${ }^{13} \mathrm{C}$ NMR and MS spectra of Janus dendrimer 4cd-----------------------------43

6. ${ }^{1} \mathrm{H}$ and ${ }^{13} \mathrm{C}$ NMR spectra of the cleaved Suzuki coupling products --------------- $45-48$

Figure S21. ${ }^{1} \mathrm{H}$ and ${ }^{13} \mathrm{C}$ NMR spectra of $\mathbf{5 a} \mathbf{a}-----------------------------------------------------------45$

Figure S22. ${ }^{1} \mathrm{H}$ and ${ }^{13} \mathrm{C}$ NMR spectra of $\mathbf{5 b}$

Figure S23. ${ }^{1} \mathrm{H}$ and ${ }^{13} \mathrm{C}$ NMR spectra of $\mathbf{5 c}$

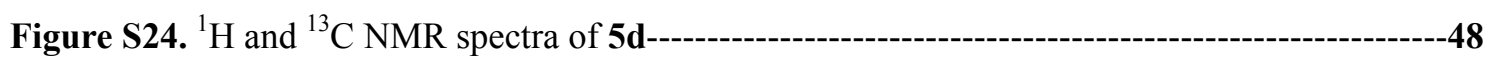

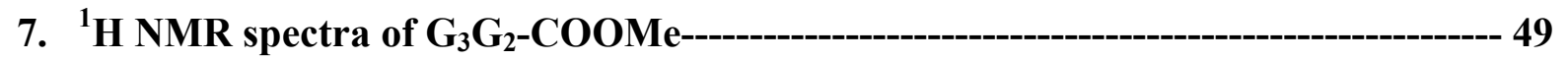

a) Before work-up

b) After precipitation by methanol and diethyl ether

8. ${ }^{1} \mathrm{H}$ NMR spectra of $4 \mathrm{ca}$ 50

a) Before work-up

b) After twice precipitation by methanol

9. References 


\section{General Information}

Unless otherwise noted, all experiments were carried out under an inert atmosphere of dry nitrogen by using standard Schlenk-type techniques. ${ }^{1} \mathrm{H}$ NMR and ${ }^{13} \mathrm{C}$ NMR spectra were recorded on a Bruker Model Avance DMX 300 Spectrometer $\left(300 \mathrm{MHz}\right.$ for ${ }^{1} \mathrm{H}, 75 \mathrm{MHz}$ for ${ }^{13} \mathrm{C}$ ). Chemical shifts are reported in parts per million (ppm) relative to the internal standard, tetramethylsilane (TMS). Data for ${ }^{1} \mathrm{H}$ NMR are reported as follows: chemical shift, integration, multiplicity s) singlet, d) doublet, t) triplet, q) quartet, m) multiplet, br) broad and $J$, coupling constants. Matrix-assisted laser desorption-ionization (time of flight) mass spectrometry (MALDI-TOF) was performed on a BIFLEX III instrument with $\alpha$-cyano-4- hydroxylcinnamic acid (CCA) as the matrix.

Fréchet-type dendrimers were prepared according to literature procedures. ${ }^{1} \mathrm{Pd}$ catalyst $\mathrm{Pd}\left[\mathrm{P}\left(p-\mathrm{C}_{6} \mathrm{H}_{4} \mathrm{CH}_{3}\right)_{3}\right]_{3}$ was synthesized according to literature procedures. ${ }^{2}$ THF and 1, 4dioxane were distilled from sodium/benzophenone and degassed three times prior to use. All other chemicals were obtained from Aldrich or Acros and used as received unless otherwise mentioned.

\section{General procedure for the synthesis of Janus dendrimers}

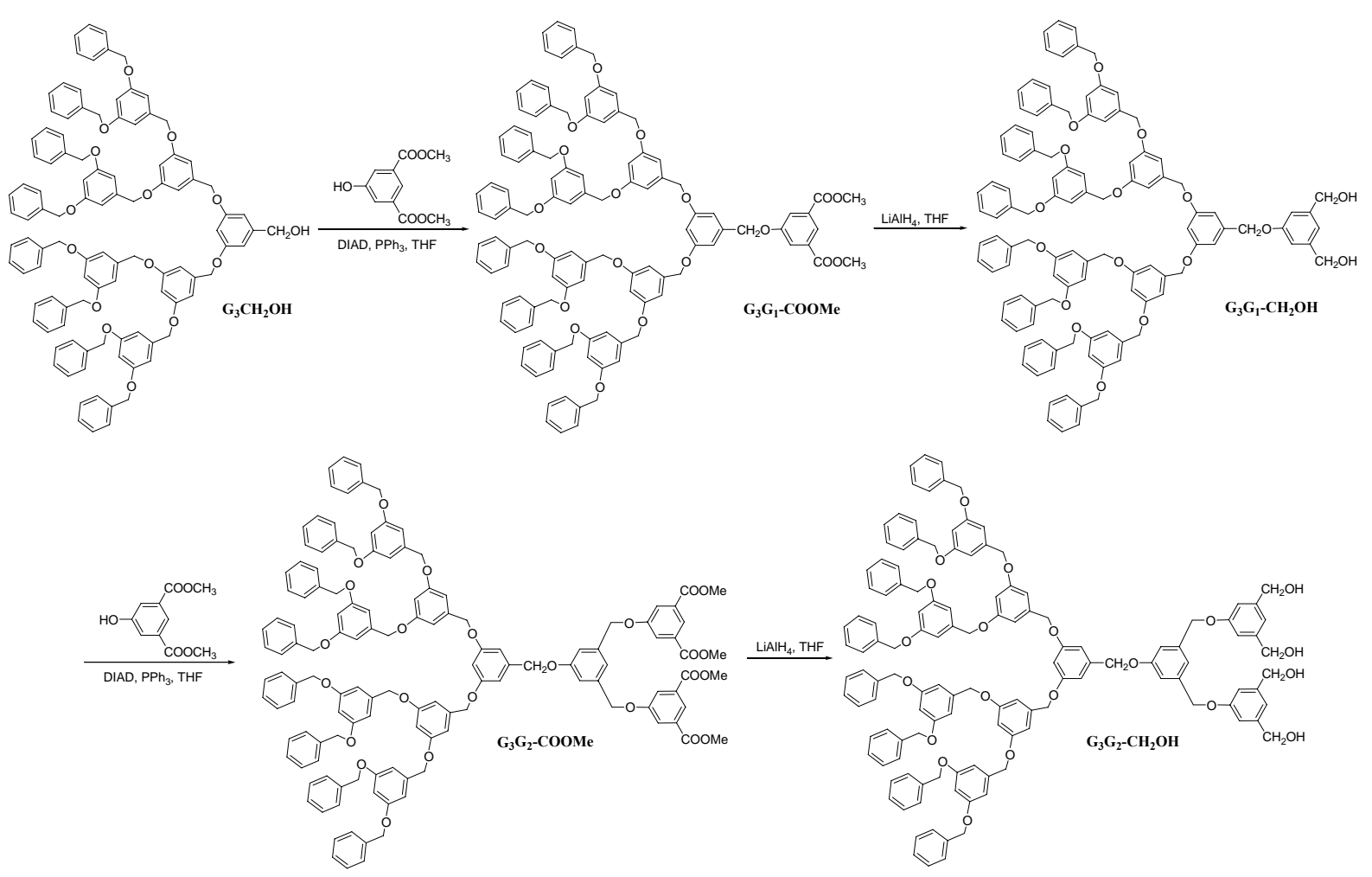




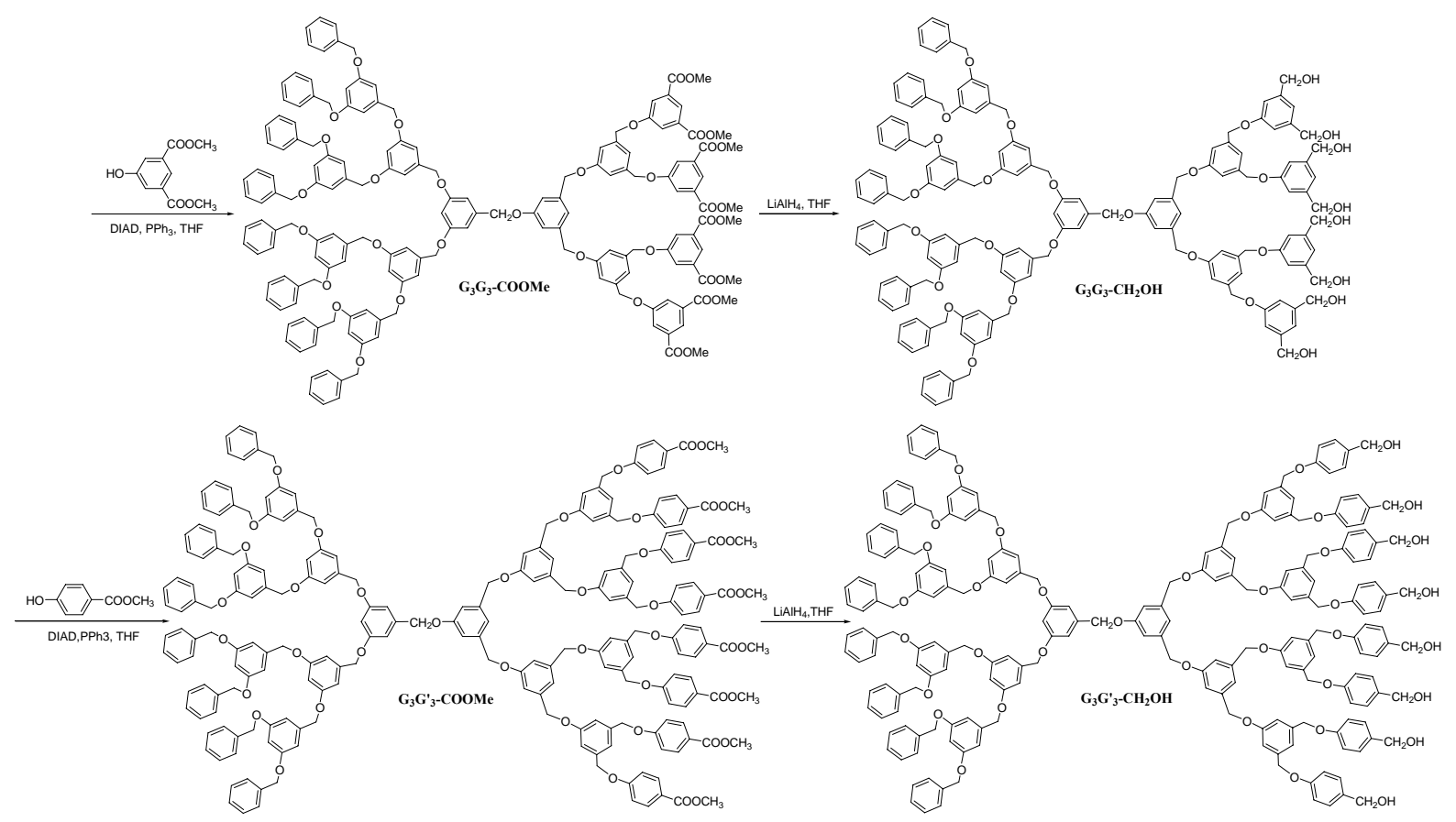

Scheme 1. Synthetic route to Janus dendrimers

\section{General Procedure for the Mitsunobu Reactions. Synthesis of $\mathbf{G}_{3} \mathbf{G}_{1}-\mathrm{COOCH}_{3}$.}

To an ice-bath cooled solution of $\mathbf{G}_{3} \mathbf{C} \mathbf{H}_{\mathbf{2}} \mathbf{O H}$ (3.660 g, $\left.2.296 \mathrm{mmol}\right), \mathbf{2}(0.531 \mathrm{~g}, 2.526$ mmol), triphenylphosphine $\left(\mathrm{PPh}_{3}, 0.663 \mathrm{~g}, 2.526 \mathrm{mmol}\right)$ and dry THF (36 mL) was added diisopropyl azodicarboxylate (DIAD, $0.51 \mathrm{~mL}, 2.526 \mathrm{mmol}$ ) dropwise via syringe. The reaction mixtures were then stirred for $10 \mathrm{~min}$ at $0{ }^{\circ} \mathrm{C}$ and $12 \mathrm{~h}$ at room temperature under a nitrogen atmosphere. The reaction progress was monitored by TLC. The crude product was purified as follows: a) The reaction mixture (about $36 \mathrm{~mL}$ ) was added to diethyl ether (420 $\mathrm{mL}$ ) under vigorous strring, and the precipitate was isolated by filtration; b) The resulting precipitate was redissolved in THF $(36 \mathrm{~mL})$, and precipitated into methanol $(360 \mathrm{~mL})$. After filtration, an off-white solid of $\mathbf{G}_{\mathbf{3}} \mathbf{G}_{\mathbf{1}}-\mathbf{C O O C H} \mathbf{C H}_{\mathbf{3}}$ was obtained (4.060 g, 99\%). ${ }^{1} \mathrm{H}$ NMR (300 $\left.\mathrm{MHz}, \mathrm{CDCl}_{3}\right) \delta=3.89\left(\mathrm{~s}, \mathrm{COOCH}_{3}, 6 \mathrm{H}\right), 4.95\left(\mathrm{~s}, \mathrm{ArCH}_{2} \mathrm{O}, 12 \mathrm{H}\right), 5.00\left(\mathrm{~s}, \mathrm{ArCH}_{2} \mathrm{O}, 16 \mathrm{H}\right)$, 5.03 (s, $\left.\mathrm{ArCH}_{2} \mathrm{O}, 2 \mathrm{H}\right), 6.53-6.56$ (m, $\left.\mathrm{ArH}, 7 \mathrm{H}\right), 6.65-6.67$ (m, $\left.\mathrm{ArH}, 14 \mathrm{H}\right), 7.29-7.41$ (m, $\mathrm{PhH}$, $40 \mathrm{H}), 7.80(\mathrm{~d}, J=1.4 \mathrm{~Hz}, \operatorname{ArH}, 2 \mathrm{H}), 8.28$ (d, $J=1.4 \mathrm{~Hz}, \operatorname{ArH}, 1 \mathrm{H}) .{ }^{13} \mathrm{C}$ NMR $(75 \mathrm{MHz}$, $\left.\mathrm{CDCl}_{3}\right) \delta=166.2,160.4,160.3,158.9,139.5,139.4,138.8,137.0,132.1,128.8,128.6,128.5$, $128.2,127.9,127.8,127.4,123.5,120.3,106.6,102.0,101.8,70.4,70.3,70.2,52.6$. MS 
(MALDI-TOF): $\mathrm{m} / \mathrm{z}$ Calcd for $\mathrm{C}_{115} \mathrm{H}_{100} \mathrm{O}_{19}: 1785.7$. found $1808.3\left[\mathrm{M}^{+}+\mathrm{Na}\right], 1824.3\left[\mathrm{M}^{+}+\mathrm{K}\right]$.

$\mathbf{G}_{3} \mathbf{G}_{2}-\mathbf{C O O C H}$. Following the procedure for $\mathbf{G}_{3} \mathbf{G}_{\mathbf{1}}-\mathbf{C O O C H}, \mathbf{G}_{3} \mathbf{G}_{1}-\mathbf{C H}_{2} \mathbf{O H}(2.560 \mathrm{~g}$, $1.480 \mathrm{mmol}), 2$ (0.777 g, $3.699 \mathrm{mmol}), \mathrm{PPh}_{3}$ (0.970 g, $\left.3.699 \mathrm{mmol}\right)$, DIAD (3.699 mmol, $0.74 \mathrm{~mL})$ and dry THF (30 mL) yielded $\mathbf{G}_{\mathbf{3}} \mathbf{G}_{\mathbf{2}}-\mathbf{C O O C H} \mathbf{C O}_{3}$ as an off-white solid (3.066 $\left.\mathrm{g}, 98 \%\right)$ after twice precipitation by diethyl ether $(300 \mathrm{~mL})$ and methanol $(260 \mathrm{~mL}) .{ }^{1} \mathrm{H}$ NMR (300 $\left.\mathrm{MHz}, \mathrm{CDCl}_{3}\right) \delta=3.89\left(\mathrm{~s}, \mathrm{COOCH}_{3}, 12 \mathrm{H}\right), 4.94\left(\mathrm{~s}, \mathrm{ArCH}_{2} \mathrm{O}, 8 \mathrm{H}\right), 4.95\left(\mathrm{~s}, \mathrm{ArCH}_{2} \mathrm{O}, 6 \mathrm{H}\right)$, 4.99 (s, $\left.\mathrm{ArCH}_{2} \mathrm{O}, 16 \mathrm{H}\right), 5.07$ (m, $\left.\mathrm{ArH}, 4 \mathrm{H}\right), 6.53-6.56$ (m, $\left.\mathrm{ArH}, 7 \mathrm{H}\right), 6.65-6.68$ (m, ArH, 14H), 7.01 (s, ArH, 2H), 7.09 (s, ArH, 1H), 7.27-7.39 (m, PhH, 40H), 7.80 (d, J=1.3 Hz, ArH, 4H), $8.28(\mathrm{~d}, J=1.2 \mathrm{~Hz}, \mathrm{ArH}, 2 \mathrm{H}) .{ }^{13} \mathrm{C} \mathrm{NMR}\left(75 \mathrm{MHz}, \mathrm{CDCl}_{3}\right) \delta=166.0,160.2,160.1,159.4$, $158.7,139.3,139.2,138.3,136.9,131.9,128.6,128.4,128.0,127.8,127.6,123.4,120.1$, 118.8, 113.6, 106.5, 106.5, 101.7, 70.1, 70.0, 52.4. MS (MALDI- TOF): m/z Calcd for $\mathrm{C}_{133} \mathrm{H}_{116} \mathrm{O}_{25}:$ 2113.8. found 2136.1 [ $\left.\mathrm{M}^{+}+\mathrm{Na}\right], 2152.1\left[\mathrm{M}^{+}+\mathrm{K}\right]$.

$\mathbf{G}_{\mathbf{3}} \mathbf{G}_{\mathbf{3}}-\mathbf{C O O C H}$. Following the procedure for $\mathbf{G}_{\mathbf{3}} \mathbf{G}_{\mathbf{1}}-\mathbf{C O O C H}, \mathbf{G}_{\mathbf{3}} \mathbf{G}_{2}-\mathbf{C H}_{\mathbf{2}} \mathbf{O H}(2.150 \mathrm{~g}$, $1.074 \mathrm{mmol}), 2$ (1.015 g, $4.832 \mathrm{mmol}), \mathrm{PPh}_{3}(1.267 \mathrm{~g}, 4.832 \mathrm{mmol}), \mathrm{DIAD}$ (4.832 mmol, $0.97 \mathrm{~mL}$ ), and dry THF (35 mL) yielded $\mathbf{G}_{\mathbf{3}} \mathbf{G}_{\mathbf{3}}-\mathbf{C O O C H} \mathbf{C H}_{3}$ as an off-white solid (2.886 g, 97\%) after twice precipitation by diethyl ether $(280 \mathrm{~mL})$ and methanol $(230 \mathrm{~mL}) .{ }^{1} \mathrm{H}$ NMR $(300$ $\left.\mathrm{MHz}, \mathrm{CDCl}_{3}\right) \delta=3.86-3.93\left(\mathrm{~m}, \mathrm{COOCH}_{3}, 24 \mathrm{H}\right), 4.90$ (s, $\left.\mathrm{ArCH}_{2} \mathrm{O}, 8 \mathrm{H}\right), 4.94\left(\mathrm{~s}, \mathrm{ArCH}_{2} \mathrm{O}\right.$, 6H), 4.98 (s, $\left.\mathrm{ArCH}_{2} \mathrm{O}, 16 \mathrm{H}\right), 5.04$ (s, $\left.\mathrm{ArCH}_{2} \mathrm{O}, 4 \mathrm{H}\right), 5.06\left(\mathrm{~s}, \mathrm{ArCH}_{2} \mathrm{O}, 8 \mathrm{H}\right), 6.51-6.56$ (m, $\mathrm{ArH}, 7 \mathrm{H}), 6.64-6.68$ (m, ArH, 14H), 7.02 (s, ArH, 6H), 7.09 (s, ArH, 3H), 7.27-7.39 (m, PhH, 40H), $7.79(\mathrm{~d}, J=1.4 \mathrm{~Hz}, \operatorname{ArH}, 8 \mathrm{H}), 8.27$ (t, $J=1.3 \mathrm{~Hz}, \operatorname{ArH}, 4 \mathrm{H}) .{ }^{13} \mathrm{C}$ NMR $(75 \mathrm{MHz}$, $\left.\mathrm{CDCl}_{3}\right) \delta=166.1,166.0,160.2,160.1,159.3,158.7,139.3,138.8,138.2,136.8,131.9,128.6$, $128.3,128.0,127.5,123.4,120.1,118.8,113.6,113.5,106.4,101.6,70.1,70.1,52.4 . \mathrm{MS}$ (MALDI-TOF): $\mathrm{m} / \mathrm{z}$ Calcd for $\mathrm{C}_{169} \mathrm{H}_{148} \mathrm{O}_{37}: 2770.0$. found $2793.3\left[\mathrm{M}^{+}+\mathrm{Na}\right]$.

$\mathbf{G}_{3} \mathbf{G}^{\prime}{ }_{3}-\mathbf{C O O C H}_{3}$. Following the procedure for $\mathbf{G}_{\mathbf{3}} \mathbf{G}_{\mathbf{1}}-\mathbf{C O O C H} \mathbf{C O C}_{3}, \mathbf{G}_{3} \mathbf{G}_{3}-\mathbf{C H}_{2} \mathbf{O H}(0.630 \mathrm{~g}$, $0.247 \mathrm{mmol})$, methyl p-hydroxybenzoate $(0.376 \mathrm{~g}, 2.470 \mathrm{mmol}), \mathrm{PPh}_{3}(0.649 \mathrm{~g}, 2.470$ mmol), DIAD (2.470 mmol, $0.14 \mathrm{~mL})$, and dry THF $(6 \mathrm{~mL})$ yielded $\mathbf{G}_{\mathbf{3}} \mathbf{G}_{\mathbf{3}} \mathbf{3}^{-} \mathbf{C O O C H} \mathbf{O O C}_{3}$ as 
off-white solid $(0.579 \mathrm{~g}, 97 \%)$ after twice precipitation by diethyl ether $(100 \mathrm{~mL})$ and methanol $(80 \mathrm{~mL}) .{ }^{1} \mathrm{H}$ NMR $\left(300 \mathrm{MHz}, \mathrm{CDCl}_{3}\right) \delta=3.83\left(\mathrm{~s}, \mathrm{COOCH}_{3}, 24 \mathrm{H}\right), 4.89-4.99(\mathrm{~m}$, $\left.\mathrm{ArCH}_{2} \mathrm{O}, 58 \mathrm{H}\right), \quad 6.49-6.53(\mathrm{~m}, \mathrm{ArH}, 7 \mathrm{H}), 6.61-6.63$ (m, $\left.\mathrm{ArH}, 14 \mathrm{H}\right), 6.87-6.89$ (m, ArH, 8H), 6.91-6.92 (m, ArH, 8H), 6.95 (s, ArH, 14H), 7.02 (s, ArH, 7H), 7.27-7.37 (m, PhH, 40H), 7.91-7.95 (m, ArH, 16H). ${ }^{13} \mathrm{C}$ NMR (75 MHz, $\left.\mathrm{CDCl}_{3}\right) \delta=166.7,162.2,160.2,160.1,159.2$, $139.2,138.7,138.4,136.8,131.6,128.6,128.0,127.5,123.0,118.7,114.4,113.4,106.4$, 101.6, 70.1, 69.8, 69.7, 51.8. MS (MALDI-TOF): $\mathrm{m} / \mathrm{z}$ Calcd for $\mathrm{C}_{225} \mathrm{H}_{196} \mathrm{O}_{45}:$ 3619.3. found $3642.7\left[\mathrm{M}^{+}+\mathrm{Na}\right], 3658.7\left[\mathrm{M}^{+}+\mathrm{K}\right]$.

\section{General Procedure for the Reduction Reactions. Synthesis of $\mathrm{G}_{3} \mathrm{G}_{1}-\mathrm{CH}_{2} \mathrm{OH}$.}

To a suspension of lithium aluminum hydride (LAH, $0.159 \mathrm{~g}, 4.180 \mathrm{mmol})$ in THF was added dropwise a solution of $\mathbf{G}_{\mathbf{3}} \mathbf{G}_{\mathbf{1}}$-COOMe $(3.110 \mathrm{~g}, 1.741 \mathrm{mmol})$ in dry THF (100 $\left.\mathrm{mL}\right)$ at $0{ }^{\circ} \mathrm{C}$. The reaction mixtures were then stirred and refluxed for $2 \mathrm{~h}$. The reaction was quenched by successive dropwise addition of $0.16 \mathrm{~mL}$ of $\mathrm{H}_{2} \mathrm{O}, 0.16 \mathrm{~mL}$ of $15 \% \mathrm{NaOH}$ solution, and $0.48 \mathrm{~mL}$ of $\mathrm{H}_{2} \mathrm{O}$. At this point, $\mathrm{H}_{2}$ evolution ceased. The granular salts were filtered and washed with THF (3 x $10 \mathrm{~mL}$ ). The combined filtrate was concentrated under reduced pressure. To the resultant solid was added $50 \mathrm{~mL}$ of water. The aqueous layer was extracted with EtOAc $(3 \times 100 \mathrm{~mL})$. The organic layer was combined and dried over sodium sulfate, filtered, and evaporated to give $\mathbf{G}_{\mathbf{3}} \mathbf{G}_{\mathbf{1}}-\mathbf{C} \mathbf{H}_{\mathbf{2}} \mathbf{O H}$ (2.982 g, 99\%) as an off-white solid. ${ }^{1} \mathrm{H}$ NMR $\left(300 \mathrm{MHz}, \mathrm{CDCl}_{3}\right) \delta=1.63\left(\mathrm{~s}, \mathrm{ArCH}_{2} \mathrm{OH}, 2 \mathrm{H}\right), 4.58\left(\mathrm{~s}, \mathrm{ArCH}_{2} \mathrm{OH}, 4 \mathrm{H}\right), 4.94\left(\mathrm{~s}, \mathrm{ArCH}_{2} \mathrm{O}\right.$, 12H), 4.96 (s, $\left.\mathrm{ArCH}_{2} \mathrm{O}, 2 \mathrm{H}\right), 5.00$ (s, $\left.\mathrm{ArCH}_{2} \mathrm{O}, 16 \mathrm{H}\right), 6.51-6.56$ (m, $\left.\mathrm{ArH}, 7 \mathrm{H}\right), 6.63-6.66$ (m, $\mathrm{ArH}, 14 \mathrm{H}), 6.86$ (s, ArH, 2H), 6.90 (s, ArH, 1H), 7.29-7.40 (m, $\mathrm{PhH}, 40 \mathrm{H}) .{ }^{13} \mathrm{C}$ NMR (75 $\left.\mathrm{MHz}, \mathrm{CDCl}_{3}\right) \delta=160.2,160.1,159.2,143.0,139.6,139.4,139.3,136.9,128.6,128.1,127.6$, 117.8, 112.4, 106.5, 106.4, 101.7, 70.2, 70.1, 69.8, 65.0. MS (MALDI- TOF): m/z Calcd for $\mathrm{C}_{113} \mathrm{H}_{100} \mathrm{O}_{17}:$ 1729.7. found $1752.2\left[\mathrm{M}^{+}+\mathrm{Na}\right], 1768.1\left[\mathrm{M}^{+}+\mathrm{K}\right]$.

$\mathbf{G}_{3} \mathbf{G}_{2}-\mathbf{C H}_{2} \mathbf{O H}$. Following the procedure for $\mathbf{G}_{3} \mathbf{G}_{\mathbf{1}}-\mathbf{C H}_{\mathbf{2}} \mathbf{O H}$, LAH (0.216 g, $\left.5.704 \mathrm{mmol}\right)$ in THF $(20 \mathrm{~mL})$ and $\mathbf{G}_{3} \mathbf{G}_{2}$-COOMe $(2.680 \mathrm{~g}, 1.268 \mathrm{mmol})$ in THF $(80 \mathrm{~mL})$ yielded $\mathbf{G}_{\mathbf{3}} \mathbf{G}_{\mathbf{2}}-\mathbf{C H}_{\mathbf{2}} \mathbf{O H}(2.487 \mathrm{~g}, 98 \%)$ as an off-white solid. ${ }^{1} \mathrm{H}$ NMR $\left(300 \mathrm{MHz}, \mathrm{CDCl}_{3}\right) \delta=2.12(\mathrm{~s}$, 
$\left.\mathrm{ArCH}_{2} \mathrm{OH}, 4 \mathrm{H}\right), 4.46$ (s, $\left.\mathrm{ArCH}_{2} \mathrm{OH}, 8 \mathrm{H}\right), 4.89$ (s, $\left.\mathrm{ArCH}_{2} \mathrm{O}, 8 \mathrm{H}\right), 4.91$ (s, $\left.\mathrm{ArCH}_{2} \mathrm{O}, 6 \mathrm{H}\right), 4.94$ (s, $\left.\mathrm{ArCH}_{2} \mathrm{O}, 16 \mathrm{H}\right), 4.96$ (s, $\left.\mathrm{ArCH}_{2} \mathrm{O}, 4 \mathrm{H}\right), 6.50-6.53$ (m, $\left.\mathrm{ArH}, 7 \mathrm{H}\right), 6.60-6.63$ (m, $\left.\mathrm{ArH}, 14 \mathrm{H}\right)$, 6.76-6.77 (m, ArH, 6H), 6.88 (s, ArH, 2H), 6.96 (s, ArH, 1H), 7.26-7.38 (m, PhH, 40H). ${ }^{13} \mathrm{C}$ NMR $\left(75 \mathrm{MHz}, \mathrm{CDCl}_{3}\right) \delta=160.2,160.1,159.1,158.9,142.9,139.4,139.3,139.1,136.8$, $128.6,128.3,128.0,127.6,118.5,117.7,113.1,112.3,106.5,101.7,70.1,70.0,69.5,64.7$. MS (MALDI-TOF): m/z Calcd for $\mathrm{C}_{129} \mathrm{H}_{116} \mathrm{O}_{21}$ : 2000.8. found $2023.1\left[\mathrm{M}^{+}+\mathrm{Na}\right], 2039.0$ $\left[\mathrm{M}^{+}+\mathrm{K}\right]$.

$\mathbf{G}_{\mathbf{3}} \mathbf{G}_{\mathbf{3}}-\mathbf{C H}_{\mathbf{2}} \mathbf{O H}$. Following the procedure for $\mathbf{G}_{\mathbf{3}} \mathbf{G}_{\mathbf{1}}-\mathbf{C H}_{\mathbf{2}} \mathbf{O H}$, LAH (0.292 g, $\left.7.685 \mathrm{mmol}\right)$ in THF $(30 \mathrm{~mL})$ and $\mathbf{G}_{3} \mathbf{G}_{3}$-COOMe $(2.420 \mathrm{~g}, 0.873 \mathrm{mmol})$ in THF $(90 \mathrm{~mL})$ yielded $\mathbf{G}_{\mathbf{3}} \mathbf{G}_{\mathbf{3}}-\mathbf{C H}_{\mathbf{2}} \mathbf{O H}(2.179 \mathrm{~g}, 98 \%)$ as an off-white solid. ${ }^{1} \mathrm{H}$ NMR (300 MHz, $d_{6}$-acetone) $\delta=$ 4.21 (br s, $\left.\mathrm{ArCH}_{2} \mathrm{OH}, 8 \mathrm{H}\right), 4.54$ (s, $\left.\mathrm{ArCH}_{2} \mathrm{OH}, 16 \mathrm{H}\right), 4.91-5.04$ (m, $\left.\mathrm{ArCH}_{2} \mathrm{O}, 42 \mathrm{H}\right), 6.54-6.56$ (m, ArH, 7H), 6.67-6.68 (m, ArH, 14H), 6.87-6.89 (m, ArH, 12H), 7.01 (m, ArH, 6H), 7.10 (s, $\operatorname{ArH}, 3 \mathrm{H}), 7.24-7.39$ (m, PhH, 40H). ${ }^{13} \mathrm{C}$ NMR (75 MHz, $d_{6}$ - acetone) $\delta=161.0,160.9,160.0$, $159.8,144.8,140.6,140.3,140.0,138.2,129.3,129.1,129.0,128.6,128.5,128.2,119.7$, 118.0, 114.2, 114.0, 112.2, 107.3, 102.2, 102.1, 70.5, 70.4, 70.1, 64.6. MS (MALDI-TOF): $\mathrm{m} / \mathrm{z}$ Calcd for $\mathrm{C}_{161} \mathrm{H}_{148} \mathrm{O}_{29}: 2546.0$. found $2567.5\left[\mathrm{M}^{+}+\mathrm{Na}\right]$.

$\mathbf{G}_{3} \mathbf{G}_{\mathbf{3}^{\prime}} \mathbf{C H}_{\mathbf{2}} \mathbf{\mathbf { O H }}$. Following the procedure for $\mathbf{G}_{\mathbf{3}} \mathbf{G}_{\mathbf{1}}-\mathbf{C H}_{\mathbf{2}} \mathbf{O H}$, LAH (0.061 g, $\left.1.602 \mathrm{mmol}\right)$ in THF $(20 \mathrm{~mL})$ and $\mathbf{G}_{\mathbf{3}} \mathbf{G}_{\mathbf{3}}^{\prime}$-COOMe $(0.581 \mathrm{~g}, 0.160 \mathrm{mmol})$ in THF $(60 \mathrm{~mL})$ yielded $\mathbf{G}_{\mathbf{3}} \mathbf{G}_{\mathbf{3}}{ }^{\prime}-\mathbf{C H}_{\mathbf{2}} \mathbf{O H}(0.533 \mathrm{~g}, 98 \%)$ as an off-white solid. ${ }^{1} \mathrm{H}$ NMR (300 MHz, $d_{6}$-acetone) $\delta=$ 4.01 (br s, $\mathrm{ArCH}_{2} \mathrm{OH}, 8 \mathrm{H}$ ), 4.47-4.53 (m, $\mathrm{ArCH}_{2} \mathrm{OH}, 16 \mathrm{H}$ ), 4.87-5.08 (m, $\mathrm{ArCH}_{2} \mathrm{O}, 58 \mathrm{H}$ ), 6.53 (s, ArH, 7H), 6.65 (s, ArH, 14H), 6.85-6.87 (m, ArH, 16H), 6.97 (s, ArH, 14H), 7.06 (s, $\mathrm{ArH}, 7 \mathrm{H}), 7.17-7.30$ (m, $\mathrm{PhH}+\mathrm{ArH}, 56 \mathrm{H}), 7.34-7.36$ (m, ArH, 16H). ${ }^{13} \mathrm{C} \mathrm{NMR}(75 \mathrm{MHz}$, $d_{6}$-acetone) $\delta=161.0,160.9,160.0,158.7,140.6,140.3,140.0,138.2,135.7,129.3,129.0$, 128.6, 128.5, 119.7, 115.4, 114.0, 107.3, 102.1, 70.5, 70.4, 70.2, 64.4. MS (MALDI-TOF): $\mathrm{m} / \mathrm{z}$ Calcd for $\mathrm{C}_{217} \mathrm{H}_{196} \mathrm{O}_{37}$ : 3395.4. found $3418.8\left[\mathrm{M}^{+}+\mathrm{Na}\right], 3434.9\left[\mathrm{M}^{+}+\mathrm{K}\right]$. 


\section{Janus dendrimers as soluble supports for the Pd-catalyzed Suzuki}

\section{coupling reactions}
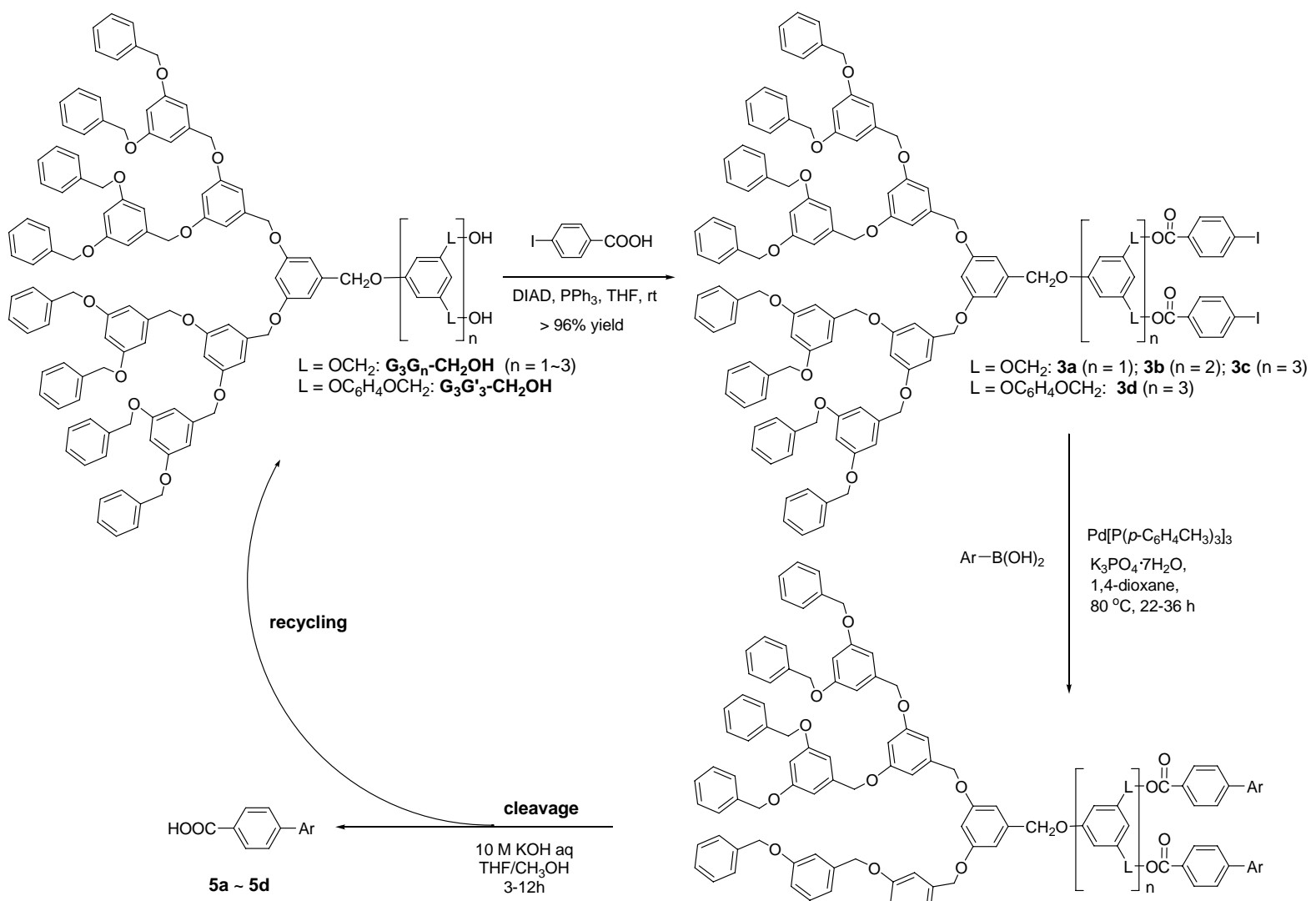

Scheme 2. The chart of Janus dendrimers as soluble supports in Suzuki coupling reactions

General Procedure for the Attachment of p-Iodobenzonic Acid to the HydroxylTerminated Janus Dendrimers. Synthesis of 3a.

To an ice-bath cooled solution of $\mathbf{G}_{3} \mathbf{G}_{\mathbf{1}}-\mathbf{C H}_{\mathbf{2}} \mathbf{O H}$ (0.476 g, $\left.0.275 \mathrm{mmol}\right)$, p-iodobenzoic acid $(0.171 \mathrm{~g}, 0.688 \mathrm{mmol}), \mathrm{PPh}_{3}(0.180 \mathrm{~g}, 0.688 \mathrm{mmol})$ and dry THF $(6 \mathrm{~mL})$ was added DIAD $(0.688 \mathrm{mmol}, 0.14 \mathrm{~mL})$ dropwise via syringe. The reaction mixtures were then stirred for $10 \mathrm{~min}$ at $0{ }^{\circ} \mathrm{C}$ and $24 \mathrm{~h}$ at room temperature under nitrogen atmosphere. The reaction progress was monitored by TLC. After successive precipitation by diethyl ether $(60 \mathrm{~mL})$ and 
methanol $(50 \mathrm{~mL})$ with the same procedure for the purification of $\mathrm{G}_{3} \mathrm{G}_{1} \mathrm{COOMe}$, an off-white solid of 3a (0.597 g, 99\%) was obtained. ${ }^{1} \mathrm{H}$ NMR (300 $\left.\mathrm{MHz}, \mathrm{CDCl}_{3}\right) \delta=4.89-5.00(\mathrm{~m}$, $\left.\mathrm{ArCH}_{2} \mathrm{O}, 30 \mathrm{H}\right), 5.27$ (s, $\left.\mathrm{CH}_{2} \mathrm{OOCAr}, 4 \mathrm{H}\right), 6.52-6.56(\mathrm{~m}, \mathrm{ArH}, 7 \mathrm{H}), 6.63-6.66(\mathrm{~m}, \mathrm{ArH}, 14 \mathrm{H})$, 6.97 (s, ArH, 2H), 7.02 (s, ArH, 1H), 7.24-7.40 (m, PhH, 40H), 7.68-7.75 (m, ArH, 8H). ${ }^{13} \mathrm{C}$ NMR $\left(75 \mathrm{MHz}, \mathrm{CDCl}_{3}\right) \delta=165.8,160.2,160.1,160.1,159.2,139.2,139.1,139.1,137.8$, 136.8, 131.1, 129.4, 128.6, 128.0, 127.5, 125.5, 120.0, 114.3, 106.5, 106.4, 101.6, 101.0, 70.1, 70.0, 66.4. MS (MALDI- TOF): $\mathrm{m} / \mathrm{z}$ Calcd for $\mathrm{C}_{127} \mathrm{H}_{106} \mathrm{I}_{2} \mathrm{O}_{19}$ : 2189.6. found $2211.6\left[\mathrm{M}^{+}+\mathrm{Na}\right]$, $2227.5\left[\mathrm{M}^{+}+\mathrm{K}\right]$.

3b. Following the procedure for $\mathbf{3 a}, \mathbf{G}_{\mathbf{3}} \mathbf{G}_{\mathbf{2}}-\mathbf{C H}_{\mathbf{2}} \mathbf{O H}(0.522 \mathrm{~g}, 0.261 \mathrm{mmol})$, $p$-iodobenzoic acid (0.291 g, $1.173 \mathrm{mmol}), \mathrm{PPh}_{3}(0.308 \mathrm{~g}, 1.173 \mathrm{mmol})$, DIAD (1.173 mmol, $\left.0.23 \mathrm{~mL}\right)$, and dry THF $(8 \mathrm{~mL})$ yielded $\mathbf{3 b}$ as an off-white solid $(0.747 \mathrm{~g}, 98 \%)$ after twice precipitation by diethyl ether $(65 \mathrm{~mL})$ and methanol $(52 \mathrm{~mL}) .{ }^{1} \mathrm{H}$ NMR $\left(300 \mathrm{MHz}, \mathrm{CDCl}_{3}\right) \delta=4.93(\mathrm{~s}$, $\left.\mathrm{ArCH}_{2} \mathrm{O}, 16 \mathrm{H}\right), 4.99$ (s, $\left.\mathrm{ArCH}_{2} \mathrm{O}, 18 \mathrm{H}\right), 5.27$ (s, $\left.\mathrm{CH}_{2} \mathrm{OOCAr}, 8 \mathrm{H}\right), 6.52-6.56(\mathrm{~m}, \mathrm{ArH}, 7 \mathrm{H})$, 6.64-6.65 (m, ArH, 14H), 6.96 (s, ArH, 6H), 7.03 (s, ArH, 3H), 7.24-7.39 (m, PhH, 40H), 7.68-7.75 (m, $\mathrm{ArH}, 16 \mathrm{H}) .{ }^{13} \mathrm{C}$ NMR $\left(75 \mathrm{MHz}, \mathrm{CDCl}_{3}\right) \delta=165.8,160.2,160.1,160.1,159.3$, $159.1,139.2,139.1,138.7,137.9,137.8,136.8,131.1,129.4,128.6,128.0,127.5,120.1$, 114.3, 113.3, 106.5, 106.4, 101.6, 101.0, 70.1, 70.0, 69.8, 66.4. MS (MALDI-TOF): $\mathrm{m} / \mathrm{z}$ Calcd for $\mathrm{C}_{157} \mathrm{H}_{128} \mathrm{I}_{4} \mathrm{O}_{25}: 2921.5$. found $2943.5\left[\mathrm{M}^{+}+\mathrm{Na}\right], 2959.5\left[\mathrm{M}^{+}+\mathrm{K}\right]$.

3c. Following the procedure for $\mathbf{3 a}, \mathbf{G}_{3} \mathbf{G}_{3}-\mathbf{C H}_{2} \mathbf{O H}$ (1.246 g, $\left.0.489 \mathrm{mmol}\right)$, p-iodobenzoic acid (1.213 g, 4.890mmol), $\mathrm{PPh}_{3}(1.283 \mathrm{~g}, 4.890 \mathrm{mmol})$, DIAD (4.890 mmol, $0.98 \mathrm{~mL}$ ), and dry THF (23 mL) yielded 3c as an off-white solid (2.103 g, 98\%) after twice precipitation by diethyl ether $(150 \mathrm{~mL})$ and methanol $(125 \mathrm{~mL}) .{ }^{1} \mathrm{H}$ NMR $\left(300 \mathrm{MHz}, \mathrm{CDCl}_{3}\right) \delta=4.92-5.00$ (m, $\left.\mathrm{ArCH}_{2} \mathrm{O}, 42 \mathrm{H}\right), 5.26$ (s, $\left.\mathrm{CH}_{2} \mathrm{OOCAr}, 16 \mathrm{H}\right), 6.51-6.53$ (m, ArH, 7H), 6.63-6.64 (m, ArH, 14H), 6.95 (s, ArH, 14H), 7.02 (s, ArH, 7H), 7.25-7.37 (m, PhH, 40H), 7.66-7.73 (m, ArH, $32 \mathrm{H}) .{ }^{13} \mathrm{C} \mathrm{NMR}\left(75 \mathrm{MHz}, \mathrm{CDCl}_{3}\right) \delta=165.8,160.2,160.1,160.1,159.3,159.1,139.2,138.7$, $138.7,137.9,137.8,136.8,135.8,131.1,129.4,128.6,128.3,128.0,127.5,125.5,120.1$, 114.3, 113.3, 106.5, 106.4, 101.6, 101.0, 70.1, 70.0, 69.8, 66.4. MS (MALDI-TOF): m/z 
Calcd for $\mathrm{C}_{217} \mathrm{H}_{172} \mathrm{I}_{8} \mathrm{O}_{37}:$ 4385.4. found $4406.0\left[\mathrm{M}^{+}+\mathrm{Na}\right], 4422.0\left[\mathrm{M}^{+}+\mathrm{K}\right]$.

3d. Following the procedure for $\mathbf{3 a}, \mathbf{G}_{\mathbf{3}} \mathbf{G}^{\prime}{ }_{\mathbf{3}} \mathbf{-} \mathbf{C H}_{\mathbf{2}} \mathbf{O H}(0.412 \mathrm{~g}, 0.121 \mathrm{mmol})$, p-iodobenzoic acid (0.301 g, $1.213 \mathrm{mmol}), \mathrm{PPh}_{3}(0.318 \mathrm{~g}, 1.213 \mathrm{mmol}), \mathrm{DIAD}$ (1.213 mmol, $0.24 \mathrm{~mL})$, and dry THF ( $8 \mathrm{~mL})$ yielded 3d as an off-white solid (0.617 $\mathrm{g}, 97 \%)$ after twice precipitation by diethyl ether $(50 \mathrm{~mL})$ and methanol $(41 \mathrm{~mL}) .{ }^{1} \mathrm{H} \mathrm{NMR}\left(300 \mathrm{MHz}, \mathrm{CDCl}_{3}\right) \delta$ $=4.96-5.02\left(\mathrm{~m}, \mathrm{ArCH}_{2} \mathrm{O}, 58 \mathrm{H}\right), 5.22\left(\mathrm{~s}, \mathrm{CH}_{2} \mathrm{OOCAr}, 16 \mathrm{H}\right), 6.53(\mathrm{~s}, \mathrm{ArH}, 7 \mathrm{H}), 6.63(\mathrm{~s}, \mathrm{ArH}$, 14H), 6.90-7.04 (m, ArH, 37H), 7.29-7.35 (m, PhH + ArH, 56H), 7.69-7.71 (m, ArH, 32H). ${ }^{13} \mathrm{C} \mathrm{NMR}\left(75 \mathrm{MHz}, \mathrm{CDCl}_{3}\right) \delta=165.9,160.2,159.2,158.8,139.2,138.9,137.7,136.8,131.1$, $130.1,129.7,129.2,128.6,128.3,128.0,127.5,125.5,118.7,114.9,114.8,113.3,106.4$, 101.6, 100.7, 70.1, 69.7, 66.7. MS (MALDI-TOF): $\mathrm{m} / \mathrm{z}$ Calcd for $\mathrm{C}_{273} \mathrm{H}_{220} \mathrm{I}_{8} \mathrm{O}_{45}$ : 5235.7. found $5258.0\left[\mathrm{M}^{+}+\mathrm{Na}\right]$.

\section{General Procedure for the Suzuki Coupling Reaction of Janus Dendrimers Supported} Iodobenzate with Aryboronic Acids. Synthesis of 4aa.

A 10-mL round-bottomed flask was charged with Janus dendrimer supported iodobenzate 3a (300.8 mg, $0.1374 \mathrm{mmol})$, phenylboronic acid (50.2 $\mathrm{mg}, 1.648 \mathrm{mmol}), \mathrm{K}_{3} \mathrm{PO}_{4} \cdot 7 \mathrm{H}_{2} \mathrm{O}$ (185.9 mg, $0.549 \mathrm{mmol}), \mathrm{Pd}$ catalyst $\mathrm{Pd}\left[\mathrm{P}\left(p-\mathrm{C}_{6} \mathrm{H}_{4} \mathrm{CH}_{3}\right)_{3}\right]_{3}(2.8 \mathrm{mg}, 0.003 \mathrm{mmol})$ and $5 \mathrm{~mL}$ degassed 1,4-dioxane. The mixtures were then stirred vigorously for $22 \mathrm{~h}$ at $80{ }^{\circ} \mathrm{C}$ under nitrogen atmosphere. Upon completion of the reaction, the solid inorganic salt was removed by filtration and washed with THF $(3 \times 8 \mathrm{~mL})$. The combined filtrate was evaporated under reduced pressure, and the resulting residue was dissolved in $\mathrm{CH}_{2} \mathrm{Cl}_{2}(20 \mathrm{~mL})$. After the small amount of insoluble inorganic salt was removed again by filtration, the filtrate was concentrated to about $3 \mathrm{~mL}$. According to the same procedure described above, the crude product was purified through twice precipitation by methanol $(35 \mathrm{~mL} \times 2)$, giving an off-white solid of $4 \mathbf{a a}(278.5 \mathrm{mg}, 97 \%)$. ${ }^{1} \mathrm{H}$ NMR (300 MHz, $\left.\mathrm{CDCl}_{3}\right) \delta=4.92-5.01$ (m, $\left.\mathrm{ArCH}_{2} \mathrm{O}, 30 \mathrm{H}\right), 5.34$ (s, $\left.\mathrm{CH}_{2} \mathrm{OOCAr}, 4 \mathrm{H}\right), 6.52-6.54$ (m, $\left.\mathrm{ArH}, 7 \mathrm{H}\right), 6.64-6.68$ (m, $\left.\mathrm{ArH}, 14 \mathrm{H}\right)$, 7.02 (s, ArH, 2H), 7.12 (s, ArH, 1H), 7.23-7.42 (m, $\mathrm{PhH}, 46 \mathrm{H})$, 7.54-7.62 (m, ArH, 8H), 8.10 $(\mathrm{d}, J=7.6 \mathrm{~Hz}, \mathrm{ArH}, 4 \mathrm{H}) .{ }^{13} \mathrm{C} \mathrm{NMR}\left(75 \mathrm{MHz}, \mathrm{CDCl}_{3}\right) \delta=166.2,160.2,160.1,159.2,145.8$, 
$139.9,139.3,138.2,136.8,130.2,128.9,128.8,128.6,128.1,128.0,127.5,127.2,127.1$, 119.9, 114.2, 106.5, 106.4, 101.7, 70.1, 70.0, 66.3. MS (MALDI-TOF): m/z Calcd for $\mathrm{C}_{139} \mathrm{H}_{120} \mathrm{O}_{19}:$ 2092.8. found $2113.9\left[\mathrm{M}^{+}+\mathrm{Na}\right], 2129.9\left[\mathrm{M}^{+}+\mathrm{K}\right]$.

4ba. Following the procedure for 4aa, 3b (305.1 mg, $0.1044 \mathrm{mmol})$, phenylboronic acid (76.4 mg, $0.626 \mathrm{mmol}), \mathrm{Pd}$ catalyst $\mathrm{Pd}\left[\mathrm{P}\left(p-\mathrm{C}_{6} \mathrm{H}_{4} \mathrm{CH}_{3}\right)_{3}\right]_{3}(4.3 \mathrm{mg}, 0.004 \mathrm{mmol}), \mathrm{K}_{3} \mathrm{PO}_{4} \cdot 7 \mathrm{H}_{2} \mathrm{O}$ (282.6 mg, $0.835 \mathrm{mmol}$ ) and $5 \mathrm{~mL}$ degassed 1,4-dioxane yielded $4 \mathbf{b a}(278.6 \mathrm{mg}, 98 \%)$ as an off-white solid after twice precipitation by methanol (35 mL x 2). ${ }^{1} \mathrm{H}$ NMR (300 MHz, $\left.\mathrm{CDCl}_{3}\right) \delta=4.91-5.02\left(\mathrm{~m}, \mathrm{ArCH}_{2} \mathrm{O}, 34 \mathrm{H}\right), 5.32\left(\mathrm{~s}, \mathrm{CH}_{2} \mathrm{OOCAr}, 8 \mathrm{H}\right), 6.51-6.54(\mathrm{~m}, \mathrm{ArH}, 7 \mathrm{H})$, 6.62-6.65 (m, ArH, 14H), 7.00 (s, ArH, 6H), 7.08-7.11 (m, ArH, 3H), 7.24-7.41 (m, PhH, 52H), 7.53-7.60 (m, ArH, 16H), 8.07-8.10 (m, ArH, 8H). $\left.{ }^{13} \mathrm{C} \mathrm{NMR} \mathrm{(75} \mathrm{MHz,} \mathrm{CDCl}_{3}\right) \delta=$ $170.2,166.2,160.2,160.1,159.3,159.1,145.8,139.9,139.3,138.8,138.2,136.8,130.2$, $128.9,128.7,128.6,128.2,128.0,127.5,127.2,127.1,119.9,118.7,114.2,113.4,106.4$, 101.7, 70.1, 70.0, 69.9, 66.2. MS (MALDI-TOF): $\mathrm{m} / \mathrm{z}$ Calcd for $\mathrm{C}_{181} \mathrm{H}_{148} \mathrm{O}_{25}:$ 2723.1. found $2746.7\left[\mathrm{M}^{+}+\mathrm{Na}\right], 2762.6\left[\mathrm{M}^{+}+\mathrm{K}\right]$.

4ca. Following the procedure for 4aa, 3c (303.4 mg, $0.0692 \mathrm{mmol})$, phenylboronic acid (101.2 $\mathrm{mg}, \quad 0.830 \mathrm{mmol})$, $\mathrm{Pd}$ catalyst $\mathrm{Pd}\left[\mathrm{P}\left(p-\mathrm{C}_{6} \mathrm{H}_{4} \mathrm{CH}_{3}\right)_{3}\right]_{3} \quad(5.6 \mathrm{mg}, 0.006 \mathrm{mmol})$, $\mathrm{K}_{3} \mathrm{PO}_{4} \cdot 7 \mathrm{H}_{2} \mathrm{O}(374.4 \mathrm{mg}, 1.107 \mathrm{mmol})$ and $5 \mathrm{~mL}$ degassed 1,4-dioxane yielded 4ca (270.3 mg, 98\%) as an off-white solid after twice precipitation by methanol (35 $\mathrm{mL} \times 2) .{ }^{1} \mathrm{H}$ NMR (300 $\left.\mathrm{MHz}, \mathrm{CDCl}_{3}\right) \delta=4.90-5.00\left(\mathrm{~m}, \mathrm{ArCH}_{2} \mathrm{O}, 42 \mathrm{H}\right), 5.30$ (s, $\left.\mathrm{CH}_{2} \mathrm{OOCAr}, 16 \mathrm{H}\right), 6.52-6.53(\mathrm{~m}$, $\mathrm{ArH}, 7 \mathrm{H}), 6.63-6.64$ (m, ArH, 14H), 6.96-7.10 (m, ArH, 21H), 7.23-7.39 (m, PhH, 64H), 7.50-7.67 (m, ArH, 32H), 8.07 (d, $J=8.3 \mathrm{~Hz}, \operatorname{ArH}, 16 \mathrm{H}) .{ }^{13} \mathrm{C}$ NMR $\left(75 \mathrm{MHz}, \mathrm{CDCl}_{3}\right) \delta=$ $166.2,160.2,160.1,159.3,159.2,145.8,139.9,139.3,138.9,138.8,138.2,137.8,136.9$, $131.1,130.3,129.0,128.8,128.6,128.2,128.0,127.6,127.3,127.1,119.9,114.2,113.4$, 106.6, 106.5, 101.7, 70.1, 70.0, 69.9, 66.3. MS (MALDI-TOF): m/z Calcd for $\mathrm{C}_{265} \mathrm{H}_{212} \mathrm{O}_{37}$ : 3988.5. found $4011.5\left[\mathrm{M}^{+}+\mathrm{Na}\right], 4027.8\left[\mathrm{M}^{+}+\mathrm{K}\right]$.

4da. Following the procedure for 4aa, 3d $(296.0 \mathrm{mg}, 0.057 \mathrm{mmol})$, phenylboronic acid 
(82.7 mg, $0.678 \mathrm{mmol})$, $\mathrm{Pd}$ catalyst $\mathrm{Pd}\left[\mathrm{P}\left(p-\mathrm{C}_{6} \mathrm{H}_{4} \mathrm{CH}_{3}\right)_{3}\right]_{3}(4.6 \mathrm{mg}, 0.005 \mathrm{mmol}), \mathrm{K}_{3} \mathrm{PO}_{4} \cdot 7 \mathrm{H}_{2} \mathrm{O}$ (306.1 $\mathrm{mg}, 0.905 \mathrm{mmol})$ and $5 \mathrm{~mL}$ degassed 1,4-dioxane yielded 4da (268.0 mg, 98\%) as an off-white solid after twice precipitation by methanol (35 mL x 2). ${ }^{1} \mathrm{H}$ NMR (300 MHz, $\left.\mathrm{CDCl}_{3}\right) \delta=4.89-4.95\left(\mathrm{~m}, \mathrm{ArCH}_{2} \mathrm{O}, 58 \mathrm{H}\right), 5.24$ (s, $\left.\mathrm{CH}_{2} \mathrm{OOCAr}, 16 \mathrm{H}\right), 6.51(\mathrm{~s}, \mathrm{ArH}, 7 \mathrm{H})$, 6.61-6.62 (m, ArH, 14H), 6.89-7.03 (m, ArH, 37H), 7.24-7.40 (m, $\mathrm{PhH}+\mathrm{ArH}, 80 \mathrm{H})$, 7.54-7.59 (m, ArH, 32H), 8.05-8.08 (m, ArH, 16H). . ${ }^{13} \mathrm{C}$ NMR (75 MHz, $\left.\mathrm{CDCl}_{3}\right) \delta=166.3$, $160.2,159.2,158.7,145.7,140.0,139.3,139.0,138.8,136.8,130.2,130.0,129.2,128.9$, 128.6, 128.1, 128.0, 127.5, 127.3, 127.0, 118.7, 115.0, 113.3, 106.4, 101.6, 70.1, 69.8, 66.5. MS (MALDI- TOF): $\mathrm{m} / \mathrm{z}$ Calcd for $\mathrm{C}_{321} \mathrm{H}_{260} \mathrm{O}_{45}$ : 4837.5. found $4860.0\left[\mathrm{M}^{+}+\mathrm{Na}\right], 4876.0$ $\left[\mathrm{M}^{+}+\mathrm{K}\right]$.

4cb. Following the procedure for 4aa, 3c (307.2 $\mathrm{mg}, 0.070 \mathrm{mmol})$, p-methylphenylboronic acid (112.6 mg, $0.840 \mathrm{mmol}), \mathrm{Pd}$ catalyst $\mathrm{Pd}\left[\mathrm{P}\left(p-\mathrm{C}_{6} \mathrm{H}_{4} \mathrm{CH}_{3}\right)_{3}\right]_{3}(5.7$ $\mathrm{mg}, 0.006 \mathrm{mmol}), \mathrm{K}_{3} \mathrm{PO}_{4} \cdot 7 \mathrm{H}_{2} \mathrm{O}(379.1 \mathrm{mg}, 1.120 \mathrm{mmol})$ and $5 \mathrm{~mL}$ degassed 1,4-dioxane yielded $4 \mathbf{c b}(275.7 \mathrm{mg}, 96 \%)$ as an off-white solid after twice precipitation by methanol (35 $\mathrm{mL} \times 2) .{ }^{1} \mathrm{H}$ NMR $\left(300 \mathrm{MHz}, \mathrm{CDCl}_{3}\right) \delta=2.34\left(\mathrm{~s}, \mathrm{ArCH}_{3}, 24 \mathrm{H}\right), 4.90-5.00\left(\mathrm{~m}, \mathrm{ArCH}_{2} \mathrm{O}\right.$, 42H), 5.29 (s, $\left.\mathrm{CH}_{2} \mathrm{OOCAr}, 16 \mathrm{H}\right), 6.52$ (s, $\left.\mathrm{ArH}, 7 \mathrm{H}\right), 6.63-6.64$ (m, $\left.\mathrm{ArH}, 14 \mathrm{H}\right), 6.98-7.10$ (m, ArH, 21H), 7.17-7.22 (m, ArH, 16H), 7.23-7.34 (m, PhH, 40H), 7.43 (d, J = 7.9 Hz, ArH, 16H), 7.55 (d, $J=8.2 \mathrm{~Hz}, \operatorname{ArH}, 16 \mathrm{H}), 8.05$ (d, $J=8.2 \mathrm{~Hz}, \operatorname{ArH}, 16 \mathrm{H}) .{ }^{13} \mathrm{C}$ NMR $(75 \mathrm{MHz}$, $\left.\mathrm{CDCl}_{3}\right) \delta=166.2,160.2,160.1,159.3,159.1,145.7,139.3,138.8,138.2,138.1,136.9,136.8$, $130.2,129.7,128.6,128.5,128.4,128.3,128.0,127.7,127.6,127.1,126.8,119.8,114.1$, 113.3, 106.4, 101.6, 70.1, 70.0, 69.8, 66.2, 21.2. MS (MALDI-TOF): m/z Calcd for $\mathrm{C}_{273} \mathrm{H}_{228} \mathrm{O}_{37}: 4100.7$. found $4123.5\left[\mathrm{M}^{+}+\mathrm{Na}\right], 4139.4\left[\mathrm{M}^{+}+\mathrm{K}\right]$.

4cc. Following the procedure for 4aa, 3c (304.5 mg, $0.069 \mathrm{mmol})$, p-acetophenylboronic acid (136.6 mg, $0.833 \mathrm{mmol}), \mathrm{Pd}$ catalyst $\mathrm{Pd}\left[\mathrm{P}\left(p-\mathrm{C}_{6} \mathrm{H}_{4} \mathrm{CH}_{3}\right)_{3}\right]_{3}(5.7 \mathrm{mg}, 0.006 \mathrm{mmol})$, $\mathrm{K}_{3} \mathrm{PO}_{4} \cdot 7 \mathrm{H}_{2} \mathrm{O}(375.8 \mathrm{mg}, 1.111 \mathrm{mmol})$ and $5 \mathrm{~mL}$ degassed 1,4- dioxane yielded 4cc (294.2 mg, 98\%) as an off-white solid after twice precipitation by methanol $(35 \mathrm{~mL} \times 2) .{ }^{1} \mathrm{H}$ NMR (300 $\left.\mathrm{MHz}, \mathrm{CDCl}_{3}\right) \delta=2.58\left(\mathrm{~s}, \mathrm{COCH}_{3}, 24 \mathrm{H}\right), 4.90-4.96\left(\mathrm{~m}, \mathrm{ArCH}_{2} \mathrm{O}, 42 \mathrm{H}\right), 5.31\left(\mathrm{~s}, \mathrm{CH}_{2} \mathrm{OOCAr}\right.$, 
16H), 6.52 (s, ArH, 7H), 6.63 (s, ArH, 14H), 6.99-7.11 (m, ArH, 21H), 7.27-7.34 (m, PhH, 40H), 7.60 (s, ArH, 32H), 7.95 (d, J=7.9 Hz, ArH, 16H), 8.08 (d, J=7.9 Hz, ArH, 16H). ${ }^{13} \mathrm{C}$ $\operatorname{NMR}\left(75 \mathrm{MHz}, \mathrm{CDCl}_{3}\right) \delta=197.4,165.9,160.2,159.2,159.1,144.3,144.2,139.2,138.8$, $138.1,136.8,136.6,130.3,129.6,129.0,128.6,128.2,128.0,127.5,127.3,127.2,119.8$, 118.7, 114.1, 113.3, 106.4, 101.6, 70.1, 70.0, 69.8, 66.3, 26.6. MS (MALDI-TOF): m/z Calcd for $\mathrm{C}_{281} \mathrm{H}_{228} \mathrm{O}_{45}$ : 4324.8. found 4347.3 [ $\left.\mathrm{M}^{+}+\mathrm{Na}\right]$, 4363.2 [ $\left.\mathrm{M}^{+}+\mathrm{K}\right]$.

4cd. Following the procedure for 4aa, 3c (301.6 mg, $0.069 \mathrm{mmol})$, p-fluorophenylboronic acid (115.4 mg, $0.825 \mathrm{mmol}), \mathrm{Pd}$ catalyst $\mathrm{Pd}\left[\mathrm{P}\left(p-\mathrm{C}_{6} \mathrm{H}_{4} \mathrm{CH}_{3}\right)_{3}\right]_{3}(5.6 \mathrm{mg}, 0.006 \mathrm{mmol})$, $\mathrm{K}_{3} \mathrm{PO}_{4} \cdot 7 \mathrm{H}_{2} \mathrm{O}(372.2 \mathrm{mg}, 1.100 \mathrm{mmol})$ and $5 \mathrm{~mL}$ degassed 1,4-dioxane yielded $4 \mathbf{c d}$ as an off-white solid (275.6 mg, 97\%) after twice precipitation by methanol (35 mL x 2). ${ }^{1} \mathrm{H}$ NMR $\left(300 \mathrm{MHz}, \mathrm{CDCl}_{3}\right) \delta=4.79-4.89\left(\mathrm{~m}, \mathrm{ArCH}_{2} \mathrm{O}, 42 \mathrm{H}\right), 5.19$ (s, $\left.\mathrm{CH}_{2} \mathrm{OOCAr}, 16 \mathrm{H}\right), 6.42-6.53$ (m, ArH, 21H), 6.88-6.99 (m, ArH, 37H), 7.09-7.23 (m, PhH, 40H), 7.32-7.45 (m, ArH, 32H), $7.95(\mathrm{~d}, J=8.2 \mathrm{~Hz}, \mathrm{ArH}, 16 \mathrm{H}) .{ }^{13} \mathrm{C} \mathrm{NMR}\left(75 \mathrm{MHz}, \mathrm{CDCl}_{3}\right) \delta=166.1,164.6,161.3,160.2$, $160.1,159.1,144.7,139.3,138.9,138.2,136.9$, 136.0, 135.9, 130.3, 128.9, 128.8, 128.8, 128.6, 128.0, 127.6, 126.9, 119.9, 116.0, 115.8, 114.2, 113.3, 106.5, 101.7, 70.1, 70.0, 69.9, 66.3. MS (MALDI-TOF): $\mathrm{m} / \mathrm{z}$ Calcd for $\mathrm{C}_{265} \mathrm{H}_{204} \mathrm{~F}_{8} \mathrm{O}_{37}$ : 4132.4. found $4155.6\left[\mathrm{M}^{+}+\mathrm{Na}\right]$, $4171.0\left[\mathrm{M}^{+}+\mathrm{K}\right]$.

\section{General Procedure for the Cleavage Reaction of Janus Dendrimer Supported Coupling Products. Recovery of $\mathrm{G}_{3} \mathrm{G}_{1}-\mathrm{CH}_{2} \mathrm{OH}$ and Synthesis of 5a.}

A round-bottom flask was charged with 4 aa $(269.1 \mathrm{mg}, 0.099 \mathrm{mmol}), 1: 1 \mathrm{v} / \mathrm{v}$ THF/ methanol $(23.4 \mathrm{~mL})$, and $\mathrm{KOH}$ aq $(10 \mathrm{M}, 0.46 \mathrm{~mL})$. The reaction mixtures were then refluxed for $3 \mathrm{~h}$. After the hydrolysis was completed (monitored by TLC), the reaction mixtures were concentrated to about $2.5 \mathrm{~mL}$ under reduced pressure. The resulting residue was precipitated in diethyl ether twice $(30 \mathrm{~mL} \times 2)$, affording the recovery of dendrimer support $\mathbf{G}_{\mathbf{3}} \mathbf{G}_{\mathbf{1}}-\mathbf{C H}_{\mathbf{2}} \mathbf{O H}$ (220.8 $\mathrm{mg}$, 99\% yield). The combined filtrate was then acidified with diluted $\mathrm{HCl} \mathrm{aq}$, and the aqueous layer was extracted with diethyl ether $(3 \times 20 \mathrm{~mL})$. The combined organic layers were dried over sodium sulfate, filtered, and evaporated to dryness, giving the coupling product 5a (49.8 mg, 98\%). 5a: ${ }^{1} \mathrm{H}$ NMR (300 MHz, d $\mathrm{d}_{6}$-DMSO) $\delta=7.28-7.41(\mathrm{~m}$, 
$\operatorname{ArH}, 3 \mathrm{H}), 7.61(\mathrm{~d}, J=7.3 \mathrm{~Hz}, \operatorname{ArH}, 2 \mathrm{H}), 7.68$ (d, $J=8.2 \mathrm{~Hz}, \operatorname{ArH}, 2 \mathrm{H}), 7.95$ (d, $J=8.2 \mathrm{~Hz}$, $\operatorname{ArH}, 2 \mathrm{H}), 12.89$ (br s, ArCOOH, 1H). ${ }^{13} \mathrm{C}$ NMR (75 MHz, d $\left.\mathrm{d}_{6} \mathrm{DMSO}\right) \delta=167.1,144.3$, $139.0,129.9,129.6,129.0,128.2,126.9,126.7$.

5a $\left(\mathbf{G}_{3} \mathbf{G}_{2}-\mathbf{C H}_{2} \mathbf{O H}\right)$. Following the procedure for the hydrolysis of $\mathbf{4 a a}, \mathbf{4 b a}(201.0 \mathrm{mg}$, $0.074 \mathrm{mmol}), 1: 1 \mathrm{v} / \mathrm{v}$ THF/methanol $(34.8 \mathrm{~mL})$ and $\mathrm{KOH}$ aq $(10 \mathrm{M}, 0.70 \mathrm{~mL})$ yielded the dendrimer support $\mathbf{G}_{\mathbf{3}} \mathbf{G}_{\mathbf{2}}-\mathbf{C H}_{\mathbf{2}} \mathbf{O H}(144.6 \mathrm{mg}, 98 \%$ ) and the coupling product $\mathbf{5 a}(56.7 \mathrm{mg}$, $97 \%)$.

5a $\left(\mathbf{G}_{3} \mathbf{G}_{3}-\mathbf{C H}_{\mathbf{2}} \mathbf{O H}\right)$. Following the procedure for the hydrolysis of $\mathbf{4 a a}$, $\mathbf{4 c a}(217.8 \mathrm{mg}$, $0.052 \mathrm{mmol}), 1: 1 \mathrm{v} / \mathrm{v} \mathrm{THF} / \mathrm{methanol}(49.3 \mathrm{~mL})$ and $\mathrm{KOH}$ aq $(10 \mathrm{M}, 0.98 \mathrm{~mL})$ yielded the dendrimer support $\mathbf{G}_{\mathbf{3}} \mathbf{G}_{\mathbf{3}}-\mathbf{C H}_{\mathbf{2}} \mathbf{O H}(130.1 \mathrm{mg}, 98 \%)$ and the coupling product 5a (81.0 $\mathrm{mg}$, $98 \%)$.

5a $\left(\mathbf{G}_{3} \mathbf{G}^{\prime}{ }_{3}-\mathbf{C H}_{2} \mathbf{O H}\right)$. Following the procedure for the hydrolysis of $4 \mathbf{a a}$, $4 \mathbf{d a}$ (158.3 $\mathrm{mg}$, $0.033 \mathrm{mmol}), 1: 1 \mathrm{v} / \mathrm{v} \mathrm{THF} / \mathrm{methanol}(30.9 \mathrm{~mL})$, and $\mathrm{KOH}$ aq $(10 \mathrm{M}, 0.61 \mathrm{~mL})$ yielded the dendrimer support $\mathbf{G}_{\mathbf{3}} \mathbf{G}^{\prime}{ }_{\mathbf{3}} \mathbf{-} \mathbf{C H}_{\mathbf{2}} \mathbf{O H}(107.6 \mathrm{mg}, 97 \%)$ and the coupling product 5a (50.2 $\mathrm{mg}$, $97 \%)$.

5b $\left(\mathbf{G}_{3} \mathbf{G}_{3}-\mathbf{C H}_{2} \mathbf{O H}\right)$. Following the procedure for the hydrolysis of $\mathbf{4 a a}, \mathbf{4 c b}(151.3 \mathrm{mg}$, $0.037 \mathrm{mmol}), 1: 1 \mathrm{v} / \mathrm{v} \mathrm{THF} / \mathrm{methanol}(34.7 \mathrm{~mL})$ and $\mathrm{KOH}$ aq $(10 \mathrm{M}, 0.69 \mathrm{~mL})$ yielded the dendrimer support $\mathbf{G}_{\mathbf{3}} \mathbf{G}_{\mathbf{3}}-\mathbf{C H}_{\mathbf{2}} \mathbf{O H}(91.9 \mathrm{mg}, 98 \%)$ and the coupling product $\mathbf{5 b}(60.6 \mathrm{mg}$, 96\%). 5b: ${ }^{1} \mathrm{H}$ NMR (300 MHz, $\mathrm{d}_{6}$-DMSO) $\delta=2.40\left(\mathrm{~s}, \mathrm{ArCH}_{3}, 3 \mathrm{H}\right), 7.34(\mathrm{~d}, J=8.0 \mathrm{~Hz}, \mathrm{ArH}$, 2H), 7.67 (d, $J=8.1 \mathrm{~Hz}, \operatorname{ArH}, 2 \mathrm{H}), 7.81$ (d, $J=8.4 \mathrm{~Hz}, \operatorname{ArH}, 2 \mathrm{H}), 8.05$ (d, $J=8.4 \mathrm{~Hz}, \operatorname{ArH}$, 2H), 12.99 (br s, ArCOOH, 1H). ${ }^{13} \mathrm{C}$ NMR (75 MHz, d 6 -DMSO) $\delta=167.1,144.2,137.7$, $136.1,129.9,129.6,129.3,126.7,126.4,20.7$.

5c $\left(\mathbf{G}_{3} \mathbf{G}_{3}-\mathbf{C H}_{2} \mathbf{O H}\right)$. Following the procedure for the hydrolysis of 4aa, 4cc (153.6 mg, $0.035 \mathrm{mmol}), 1: 1 \mathrm{v} / \mathrm{v} \mathrm{THF} / \mathrm{methanol}(33.4 \mathrm{~mL})$ and $\mathrm{KOH}$ aq $(10 \mathrm{M}, 0.66 \mathrm{~mL})$ yielded the 
dendrimer support $\mathbf{G}_{\mathbf{3}} \mathbf{G}_{\mathbf{3}}-\mathbf{C H}_{\mathbf{2}} \mathbf{O H}(88.3 \mathrm{mg}, 98 \%)$ and the coupling product $\mathbf{5 c}$ (66.6 $\mathrm{mg}$, 98\%). 5c: ${ }^{1} \mathrm{H}$ NMR (300 MHz, $\mathrm{d}_{6}$-DMSO) $\delta=2.61-2.63\left(\mathrm{~m}, \operatorname{ArCOCH}_{3}, 3 \mathrm{H}\right), 7.86-7.91(\mathrm{~m}$, $\mathrm{ArH}, 4 \mathrm{H}), 8.05-8.08$ (m, ArH, 4H), 13.03 (br s, ArCOOH, 1H). ${ }^{13} \mathrm{C}$ NMR (75 MHz, $\mathrm{d}_{6}$-DMSO) $\delta=197.5,167.0,143.3,143.0,136.3,130.4,130.0,128.9,127.2,127.1,26.7$.

5d $\left(\mathbf{G}_{\mathbf{3}} \mathbf{G}_{\mathbf{3}}-\mathbf{C H}_{\mathbf{2}} \mathbf{O H}\right)$. Following the procedure for the hydrolysis of $\mathbf{4 a a}, \mathbf{4} \mathbf{c d}(148.1 \mathrm{mg}$, $0.036 \mathrm{mmol}), 1: 1 \mathrm{v} / \mathrm{v} \mathrm{THF} / \mathrm{methanol}(33.8 \mathrm{~mL})$ and $\mathrm{KOH}$ aq $(10 \mathrm{M}, 0.67 \mathrm{~mL})$ yielded the dendrimer support $\mathbf{G}_{\mathbf{3}} \mathbf{G}_{\mathbf{3}}-\mathbf{C H}_{\mathbf{2}} \mathbf{O H}(88.5 \mathrm{mg}, 97 \%)$ and the coupling product $\mathbf{5 d}(60.7 \mathrm{mg}$, 98\%). 5d: ${ }^{1} \mathrm{H}$ NMR (300 MHz, $\mathrm{d}_{6}$-DMSO) $\delta=7.29-7.37$ (m, ArH, 2H), 7.76-7.83 (m, ArH, 4H), 8.01-8.04 (m, ArH, 2H), 12.99 (br s, ArCOOH, 1H). ${ }^{13} \mathrm{C}$ NMR (75 MHz, d 6 -DMSO) $\delta=$ $167.0,163.9,160.7,143.2,135.5,135.4,129.9,129.6,129.1,129.0,126.7,116.0,115.7$.

\section{4. ${ }^{1} \mathrm{H}$ and ${ }^{13} \mathrm{C}$ NMR and MS spectra of Janus Dendrimers}

Figure S1. ${ }^{1} \mathrm{H}$ NMR spectra of Dendrimers $\mathbf{G}_{3} \mathbf{C H}_{2}-\mathbf{O H}$

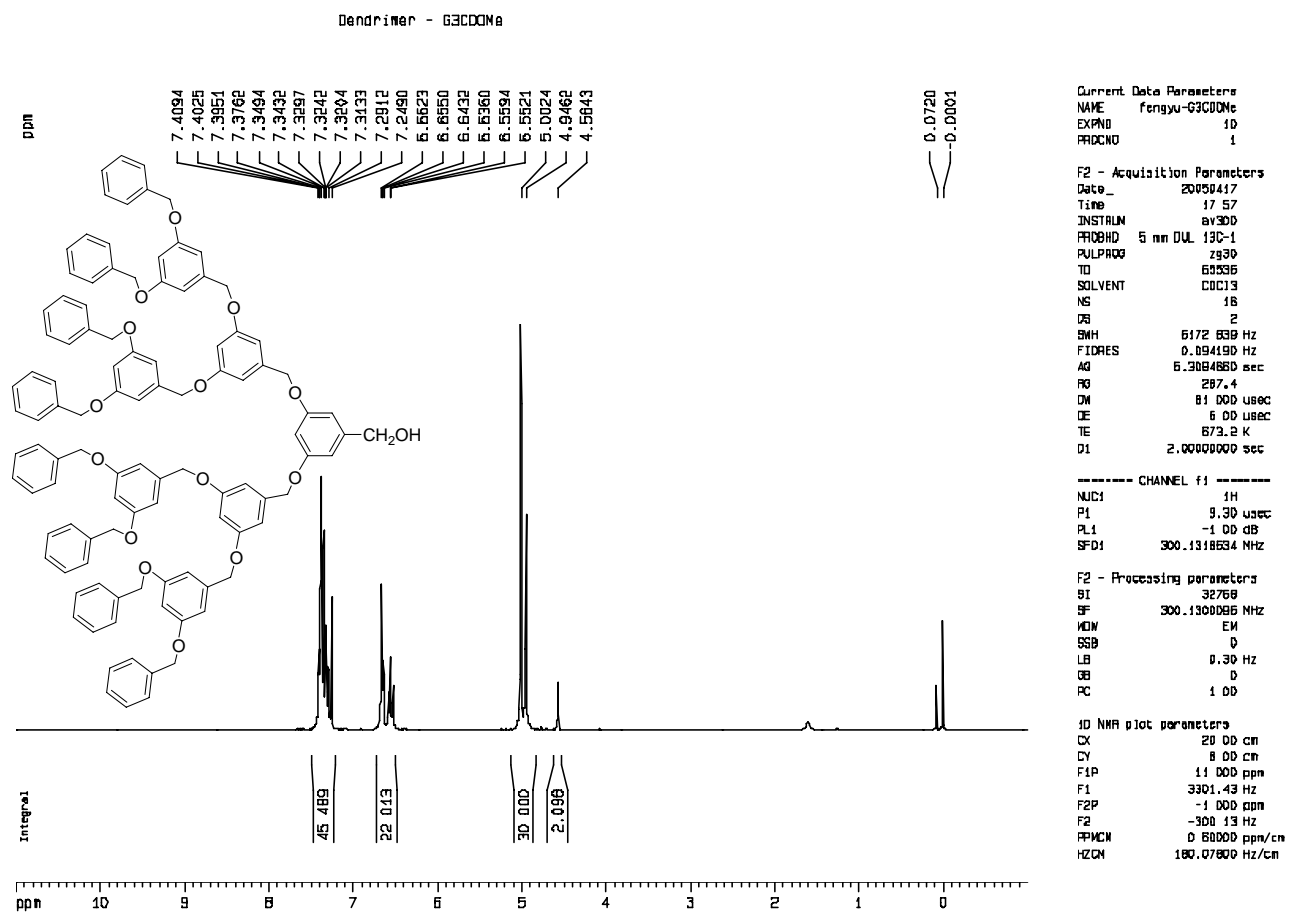


Figure S2. ${ }^{1} \mathrm{H}$ NMR, ${ }^{13} \mathrm{C}$ NMR and MS spectra of Janus Dendrimers $\mathbf{G}_{3} \mathbf{G}_{1}-\mathbf{C O O C H}$
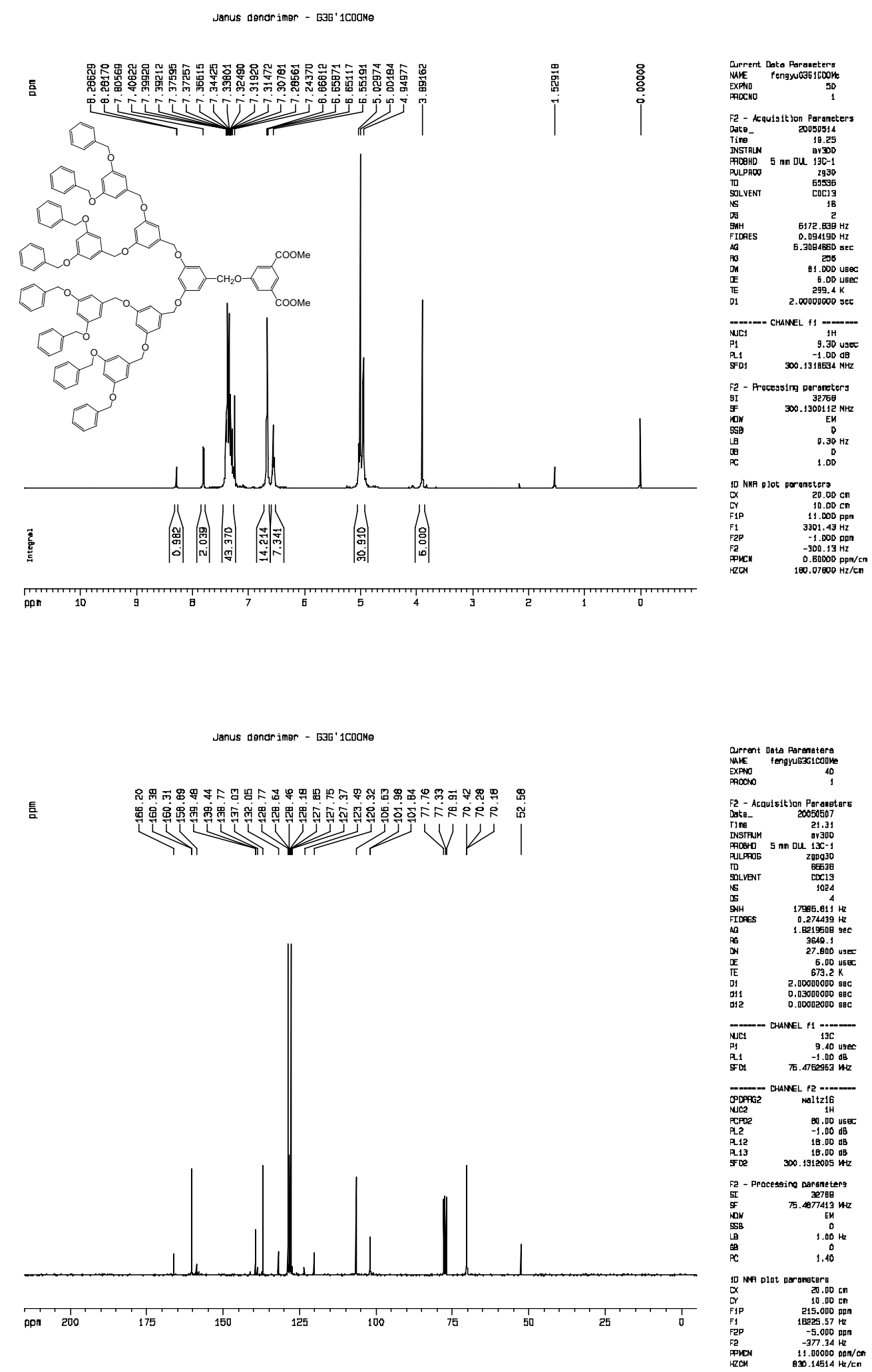
Figure S3. ${ }^{1} \mathrm{H}$ NMR, ${ }^{13} \mathrm{C}$ NMR and MS spectra of Janus Dendrimers $\mathbf{G}_{\mathbf{3}} \mathbf{G}_{\mathbf{1}}-\mathbf{C} \mathbf{H}_{\mathbf{2}} \mathbf{O H}$
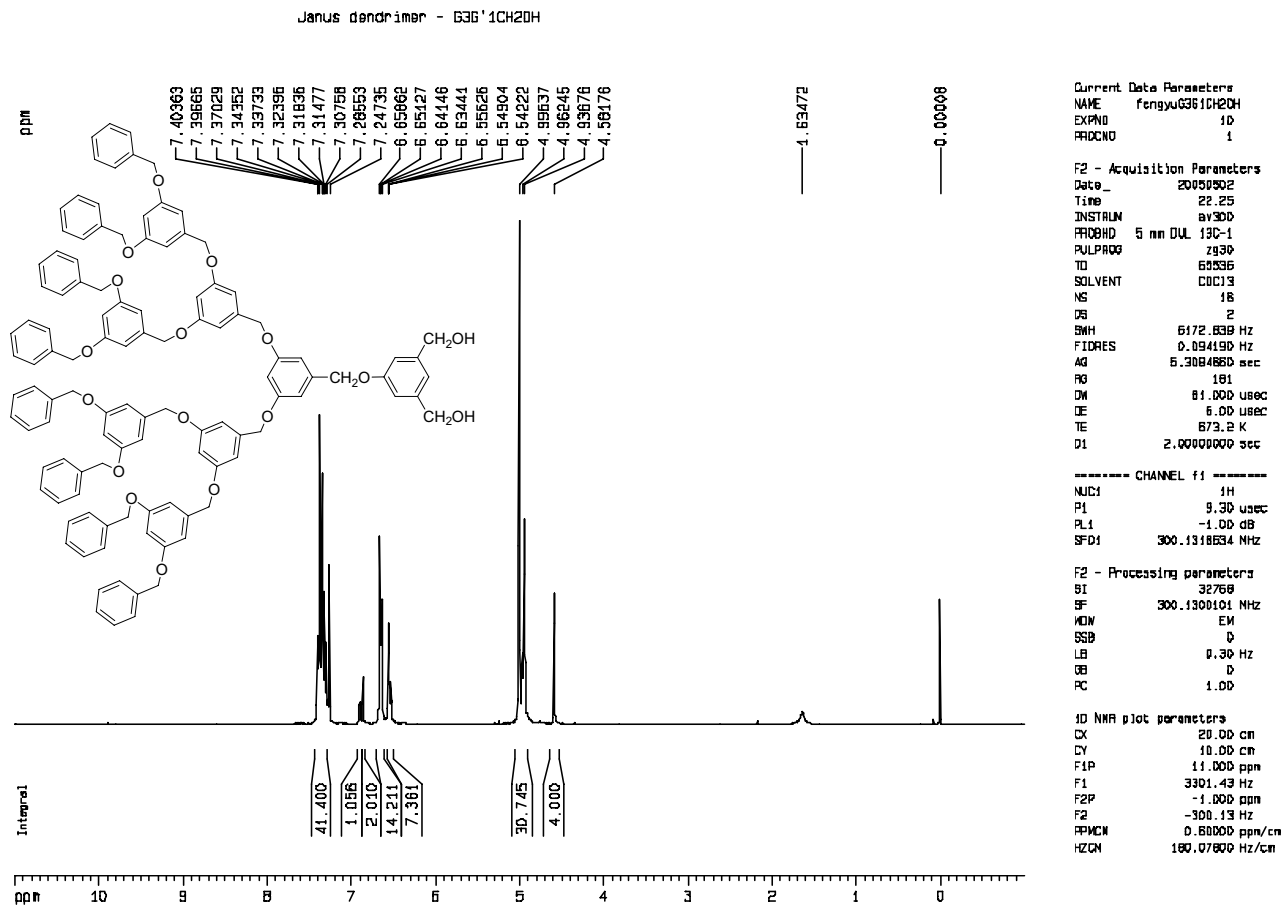
Janus dendr imer - $\mathrm{G}^{\prime} \mathrm{G}^{\prime} 1 \mathrm{CH} 2 \mathrm{OH}$
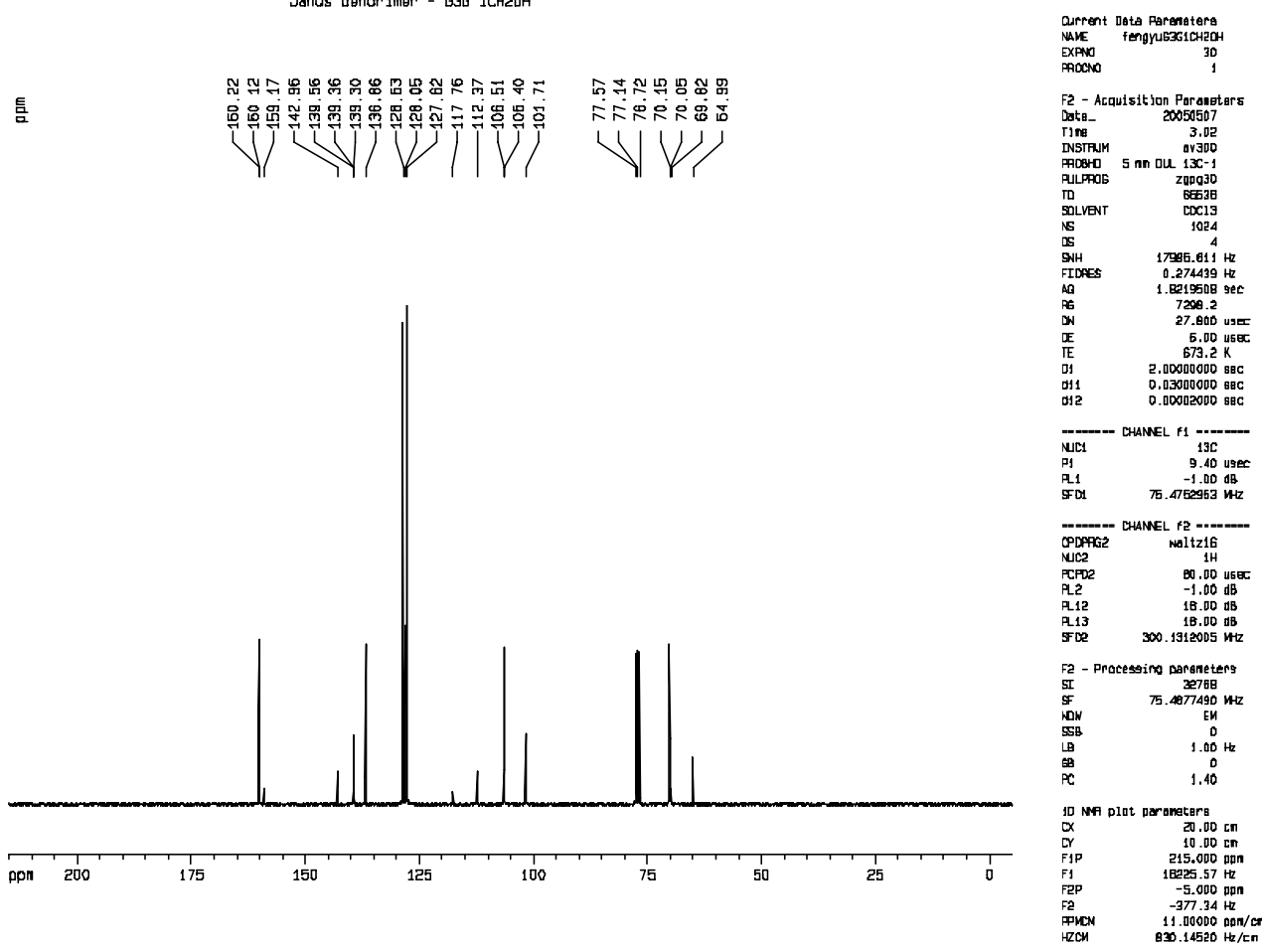

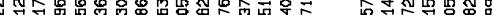

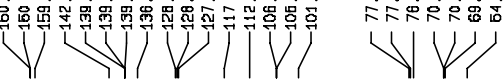

镸

MALDI - TOF , CCA, 4\#, 2005,5,9 
Figure S4. ${ }^{1} \mathrm{H}$ NMR, ${ }^{13} \mathrm{C}$ NMR and MS spectra of Janus Dendrimers $\mathbf{G}_{\mathbf{3}} \mathbf{G}_{\mathbf{2}}-\mathbf{C O O C H}$
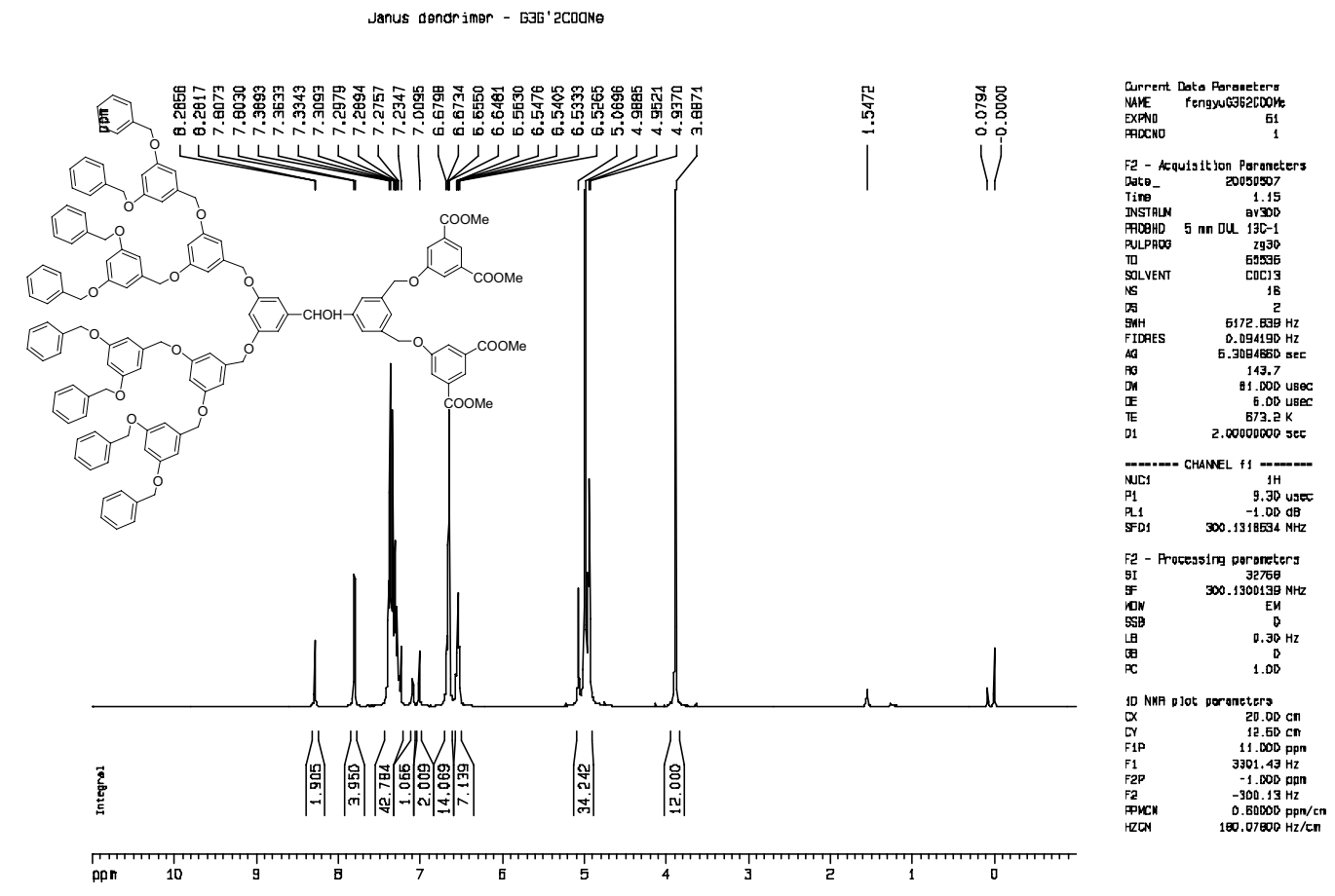

Janus dendrimer - GJG' 2CaONe
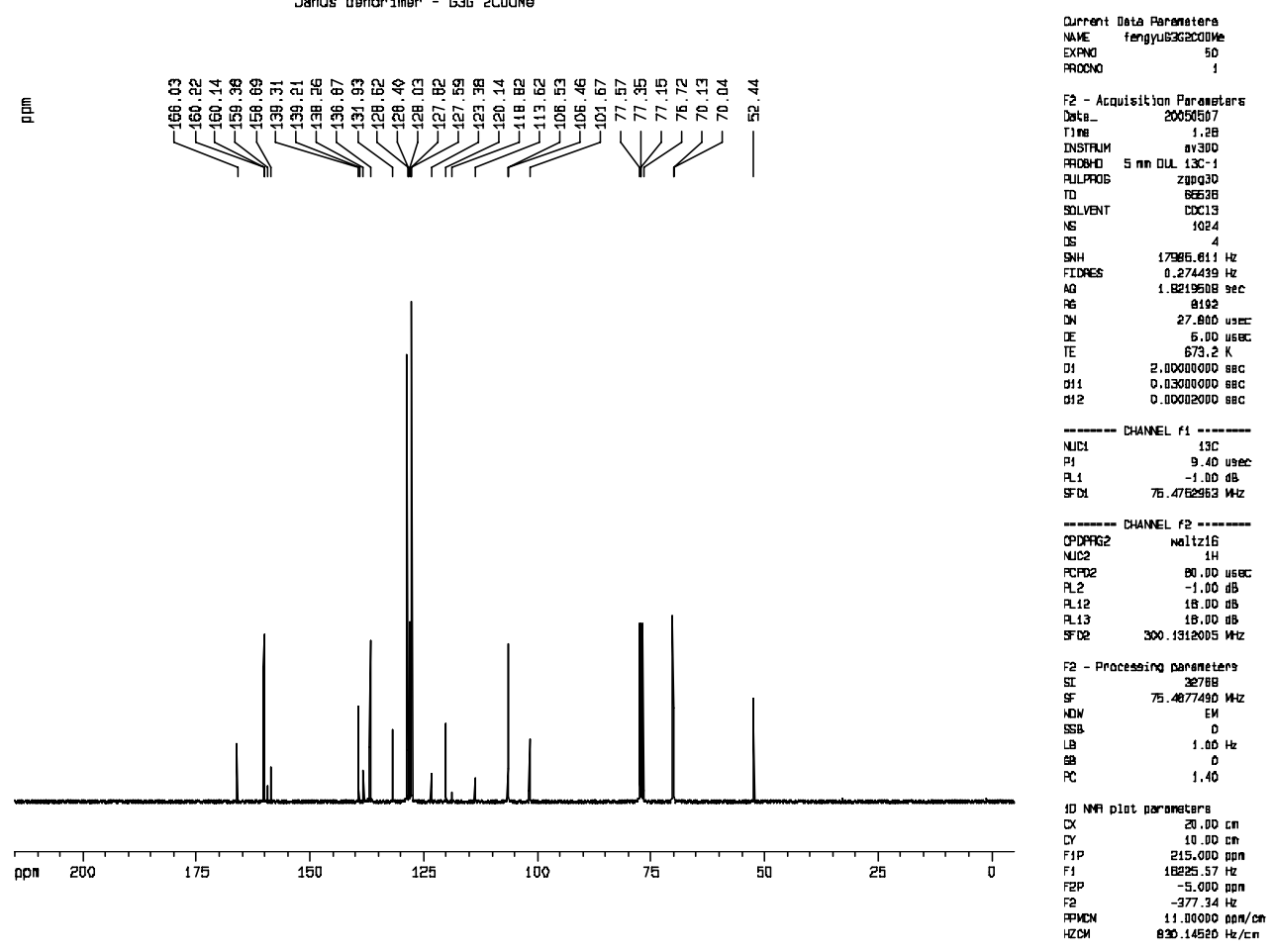
Figure S5. ${ }^{1} \mathrm{H} \mathrm{NMR},{ }^{13} \mathrm{C}$ NMR and MS spectra of Janus Dendrimers $\mathbf{G}_{\mathbf{3}} \mathbf{G}_{\mathbf{2}}-\mathbf{C} \mathbf{H}_{\mathbf{2}} \mathbf{O H}$
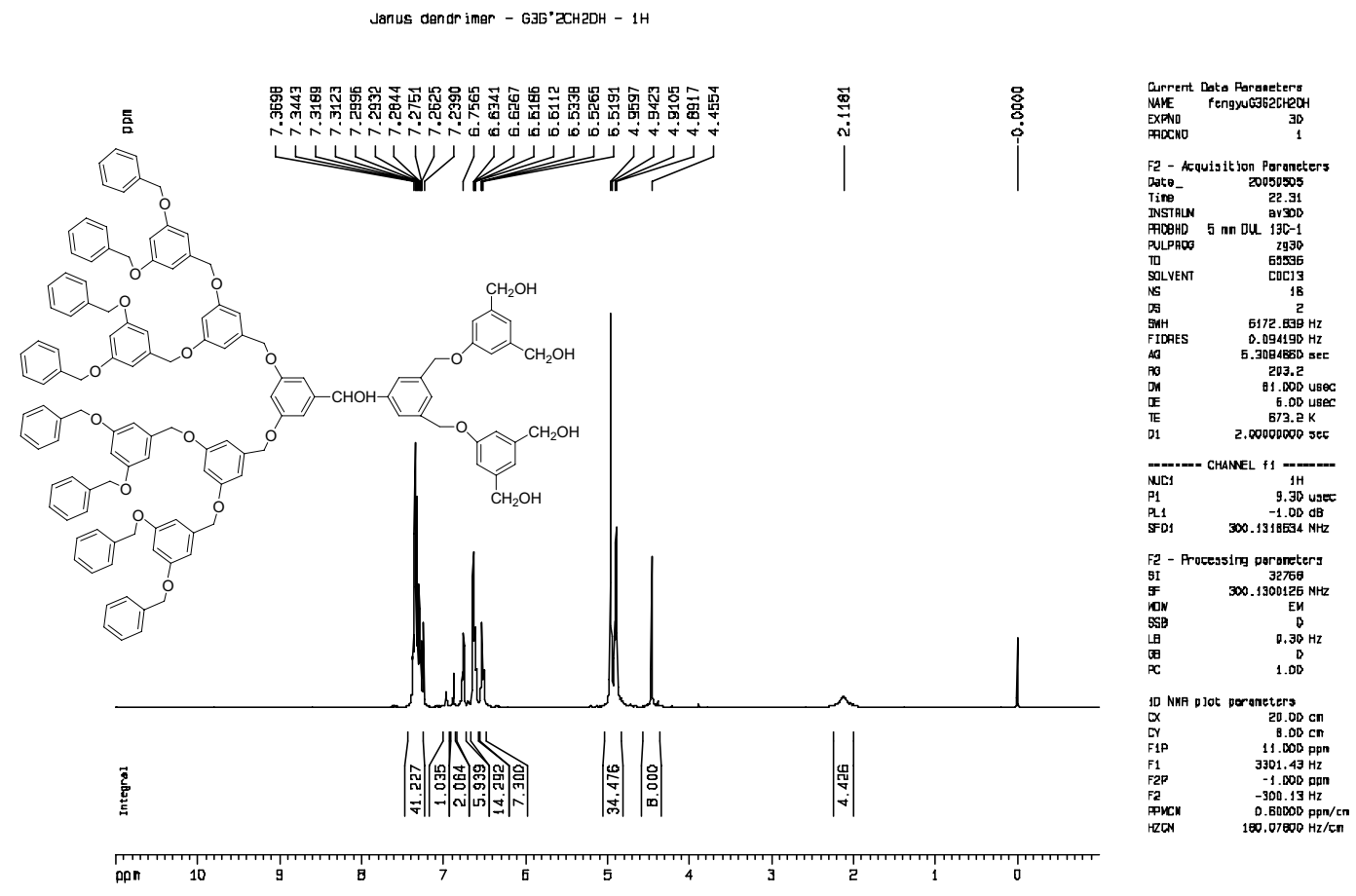
Janus dendr imer - GЭG' $2 \mathrm{CH} 2 \mathrm{OH}$
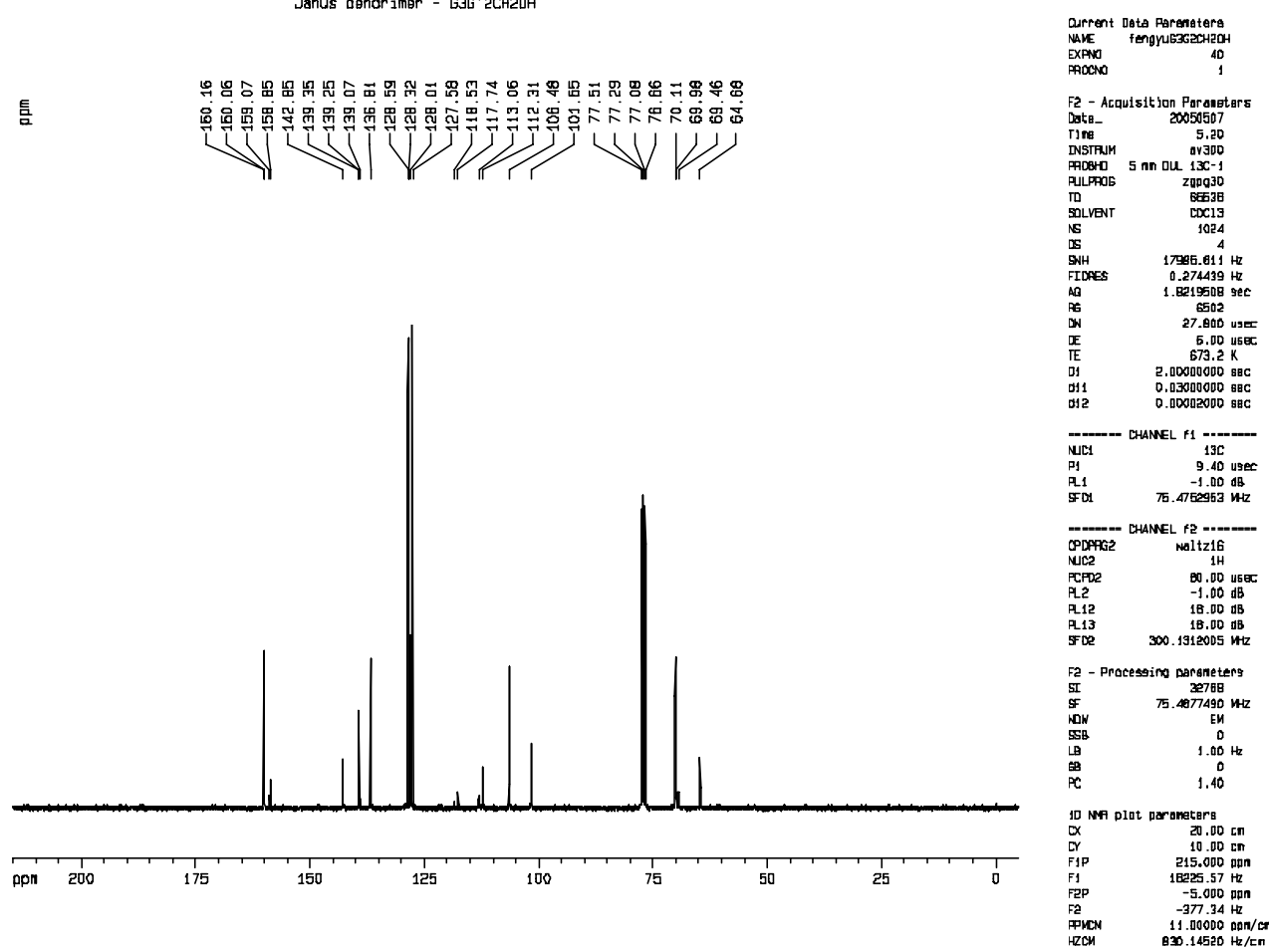

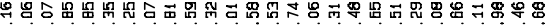

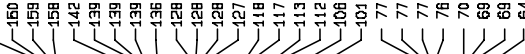

ppn 200

MALDI-TOF, CCA, $1 \#, 2005,5,8$ 
Figure S6. ${ }^{1} \mathrm{H}$ NMR, ${ }^{13} \mathrm{C}$ NMR and MS spectra of Janus Dendrimers $\mathbf{G}_{3} \mathbf{G}_{3}-\mathbf{C O O C H}_{3}$
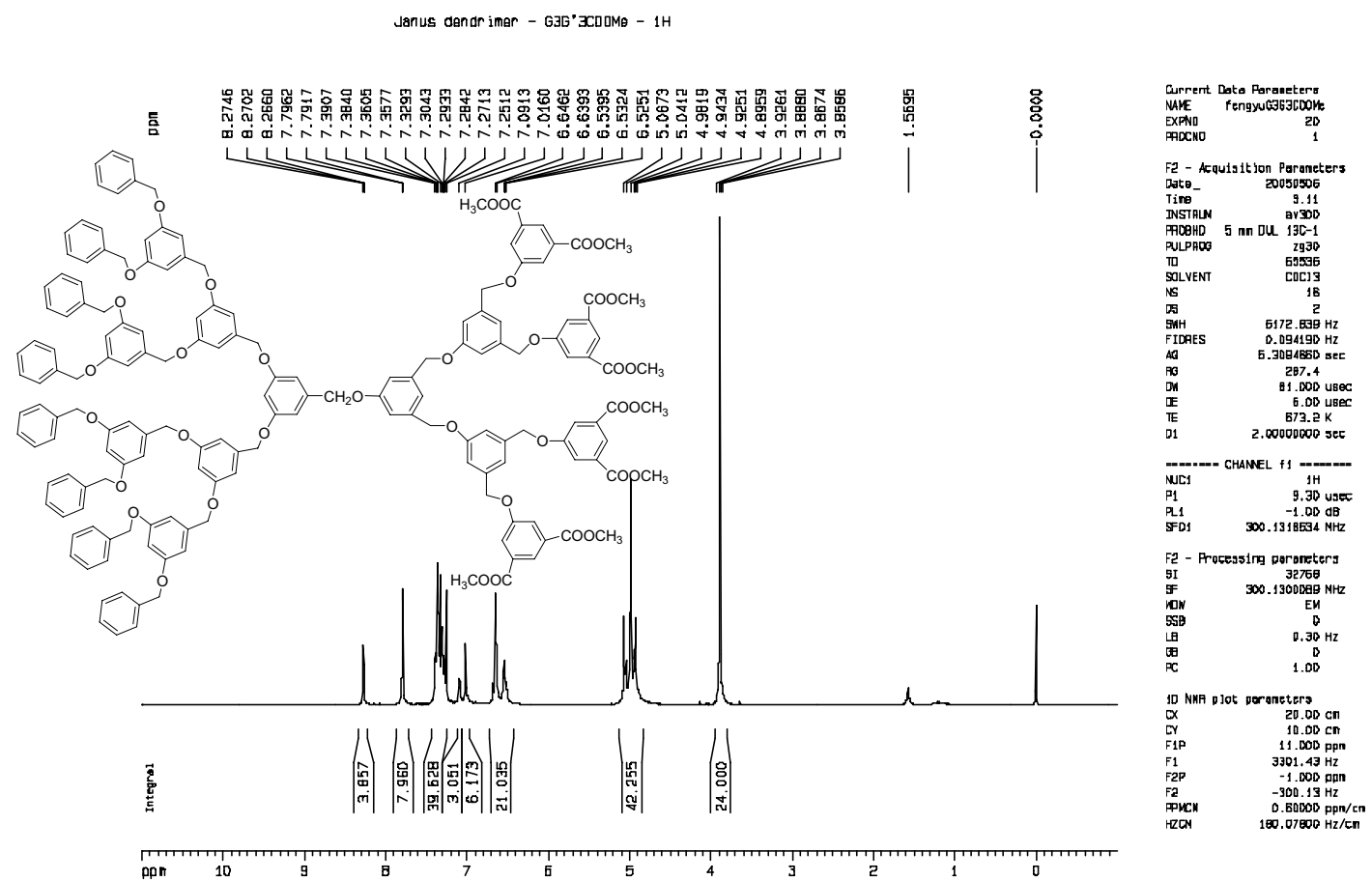

Janus dendr imer - GJG' JCDONe

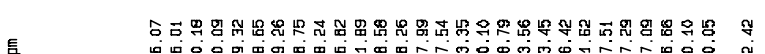

言
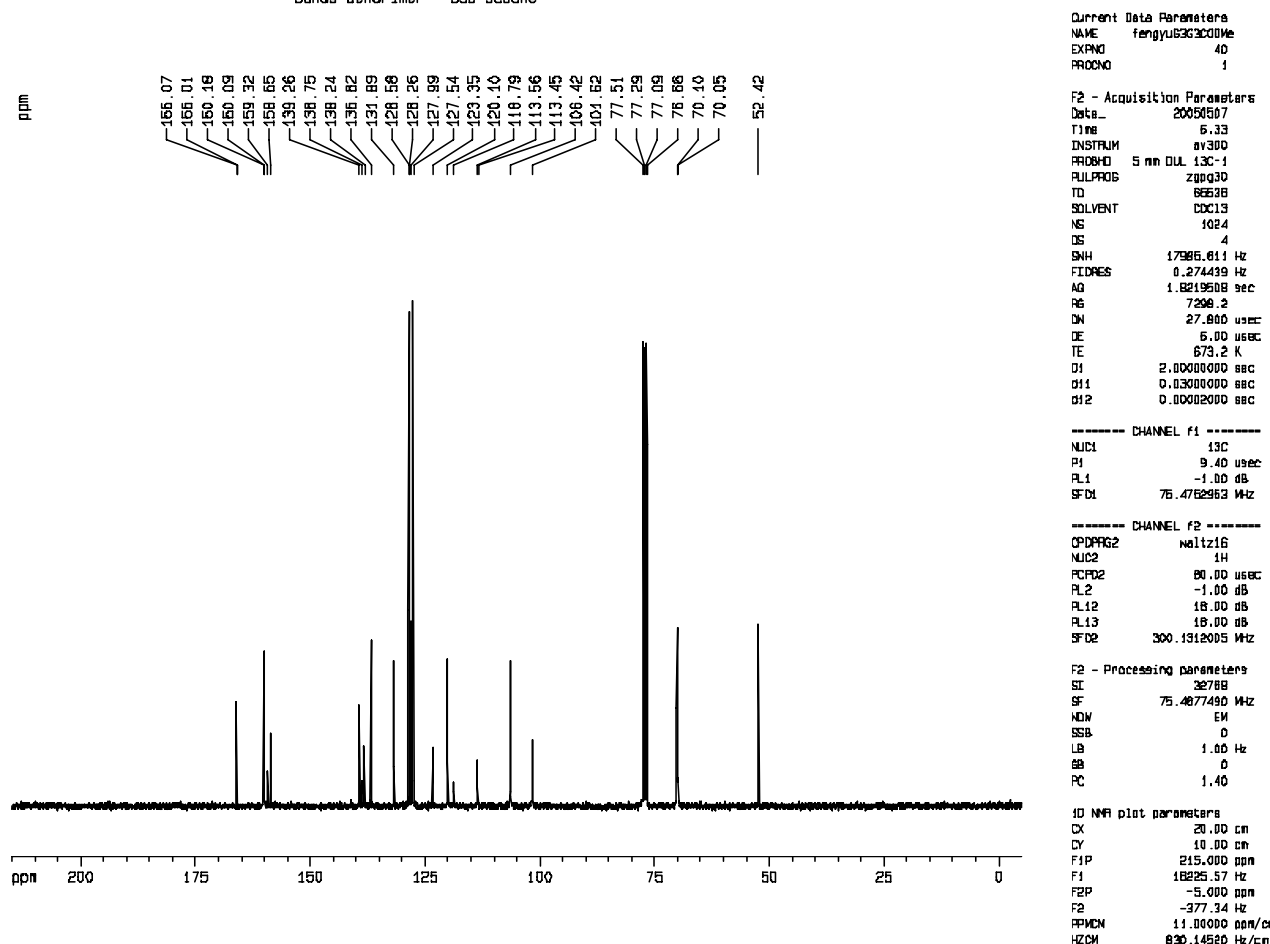


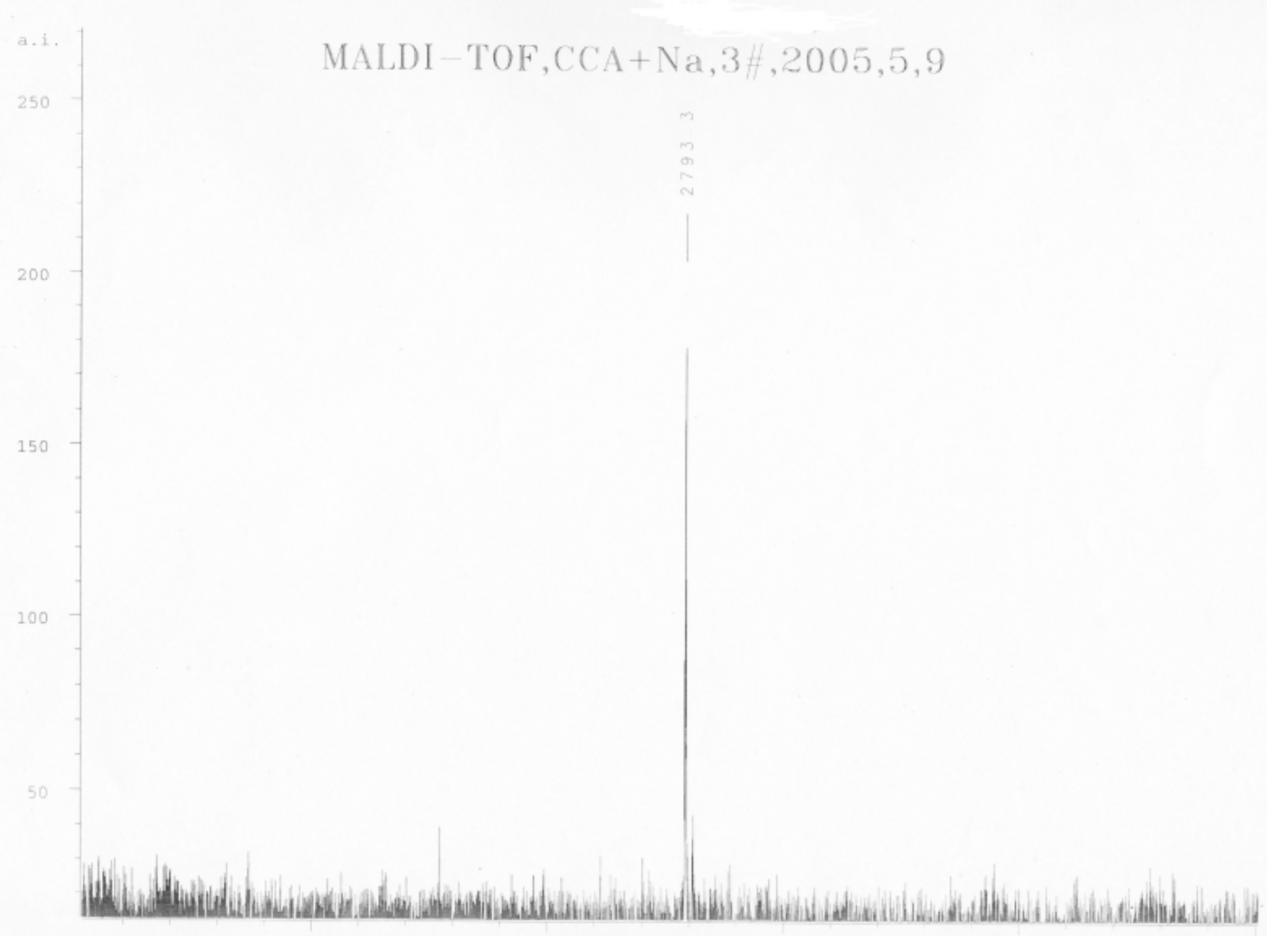

Figure S7. ${ }^{1} \mathrm{H}$ NMR, ${ }^{13} \mathrm{C}$ NMR and MS spectra of Janus Dendrimers $\mathbf{G}_{3} \mathbf{G}_{3}-\mathbf{C H}_{2} \mathbf{O H}$

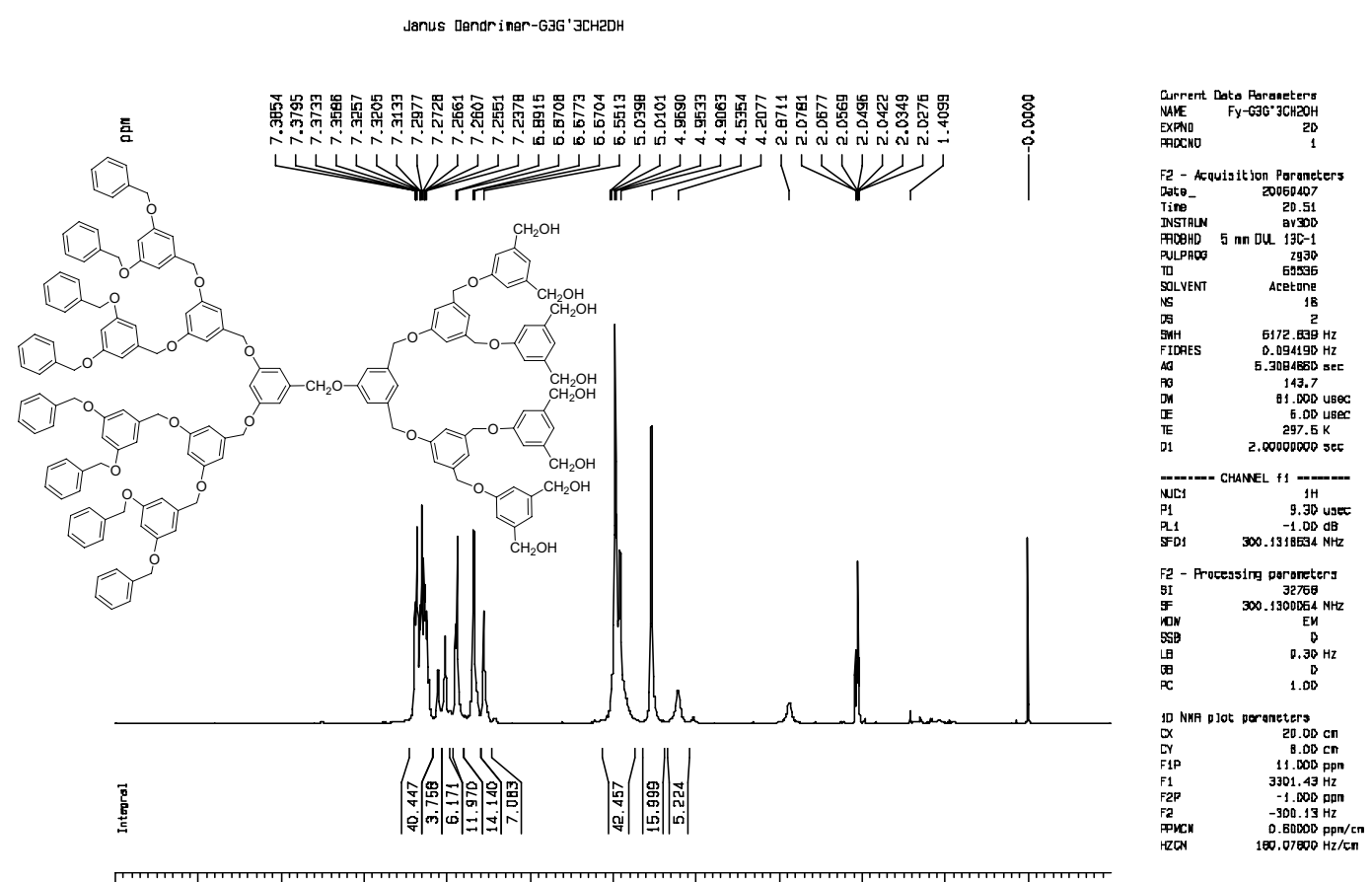



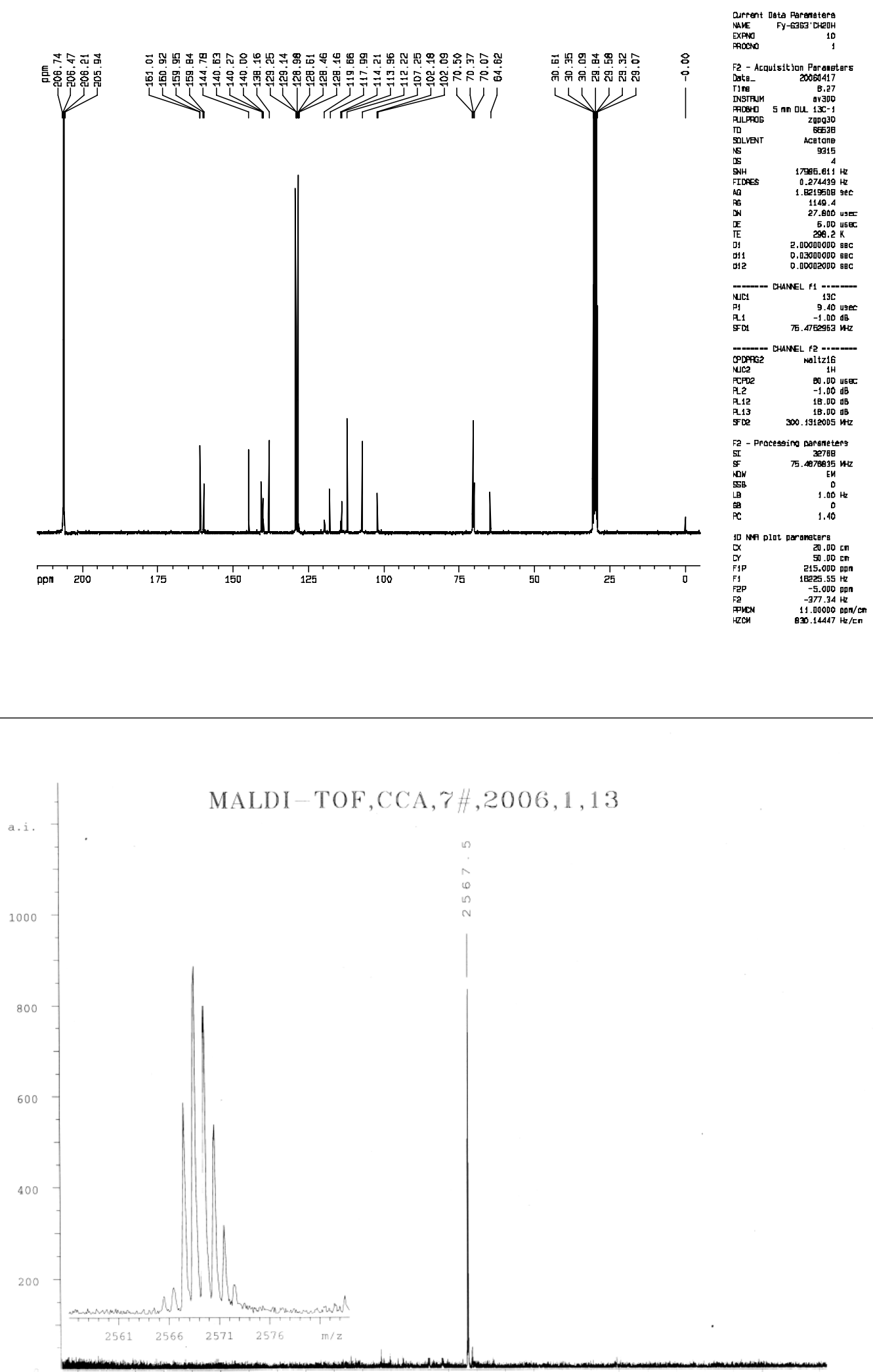
Figure S8. ${ }^{1} \mathrm{H}$ NMR, ${ }^{13} \mathrm{C}$ NMR and MS spectra of Janus Dendrimers $\mathbf{G}_{3} \mathbf{G}^{\prime}{ }_{3}-\mathbf{C O O C H} \mathbf{H}_{3}$

G3G' ' '3С00M
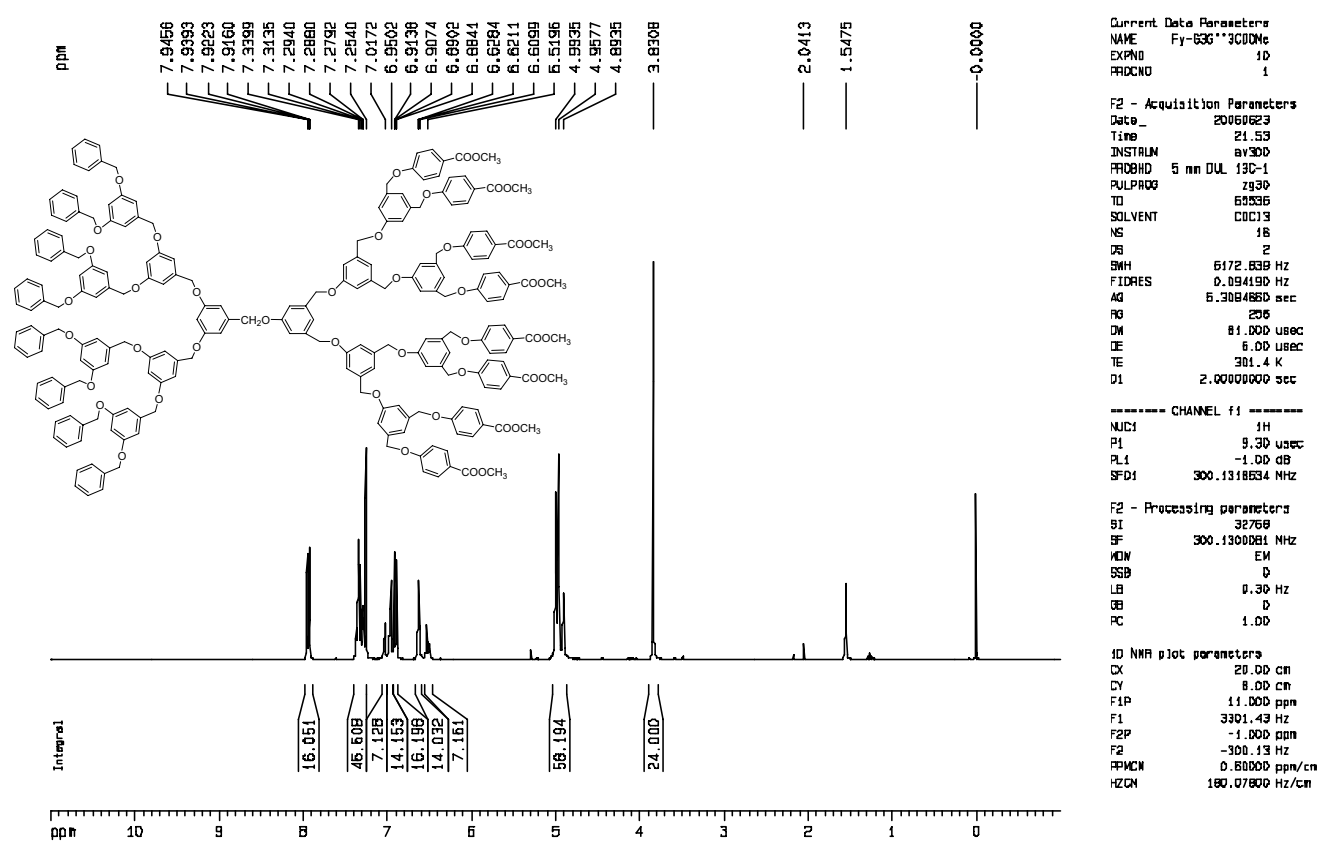

GзG' ' Зсо0Мм

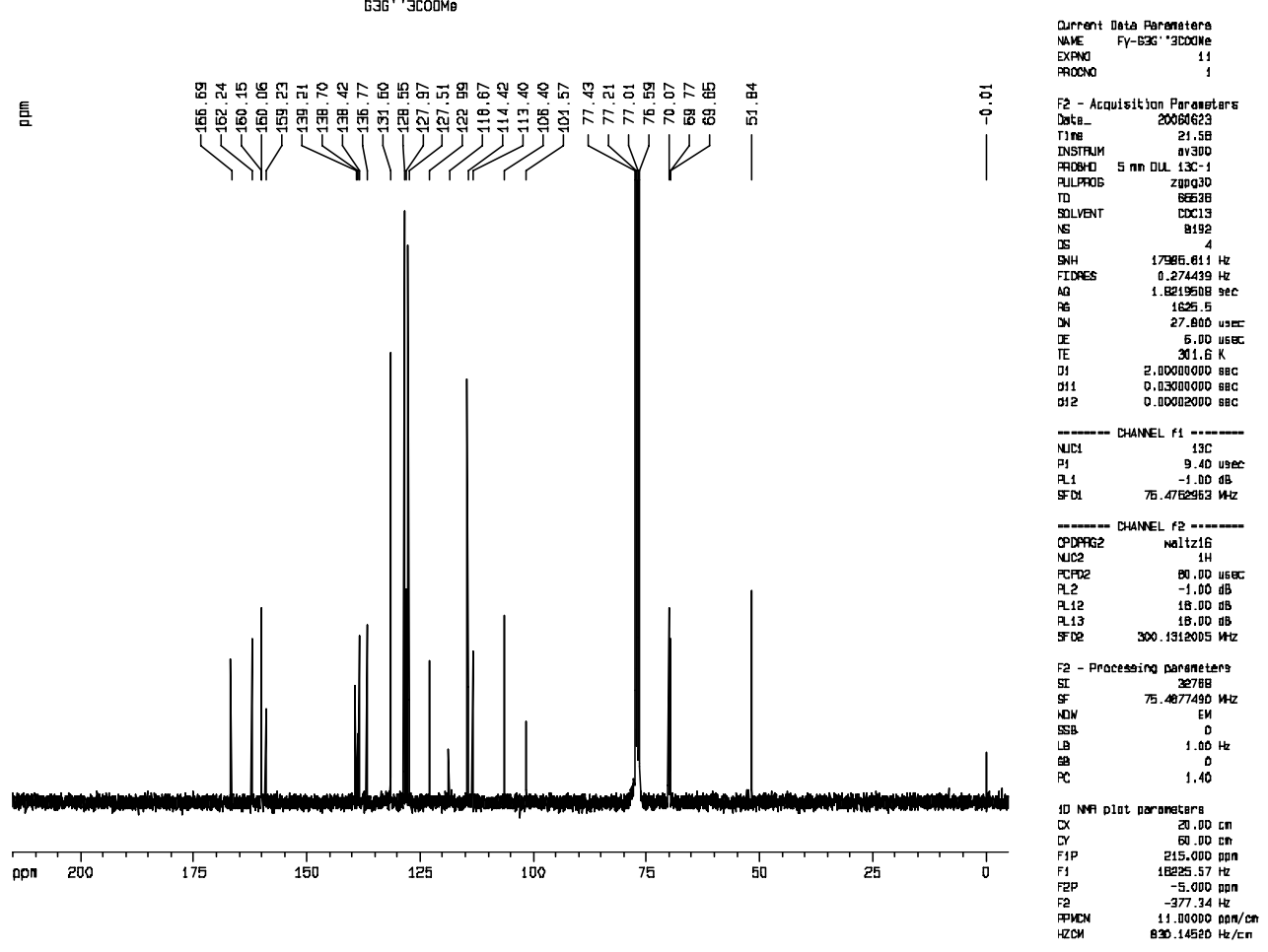




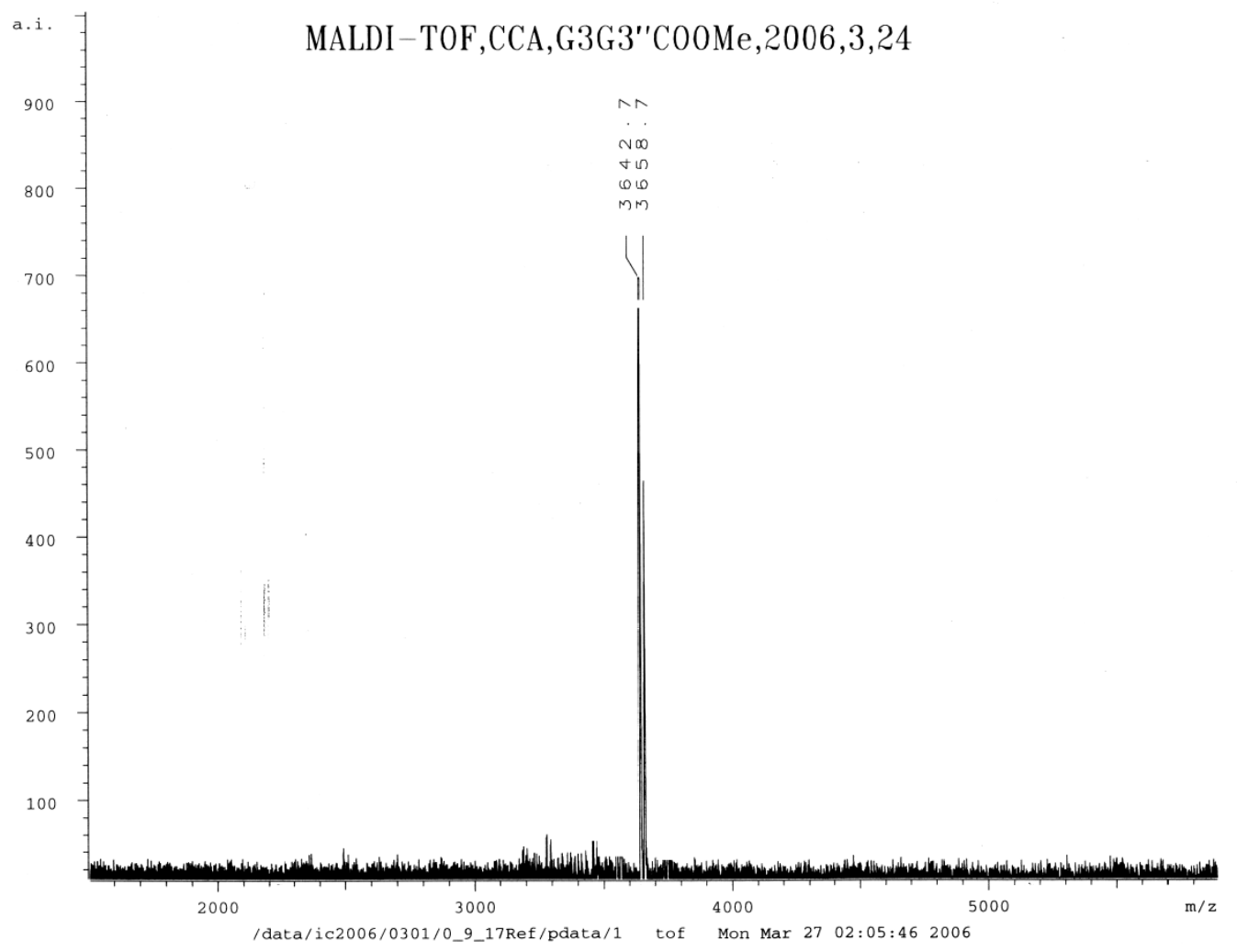

Figure S9. ${ }^{1} \mathrm{H}$ NMR, ${ }^{13} \mathrm{C}$ NMR and MS spectra of Janus Dendrimers $\mathbf{G}_{3} \mathbf{G}^{\prime}{ }_{3}-\mathbf{C H}_{\mathbf{2}} \mathbf{O H}$
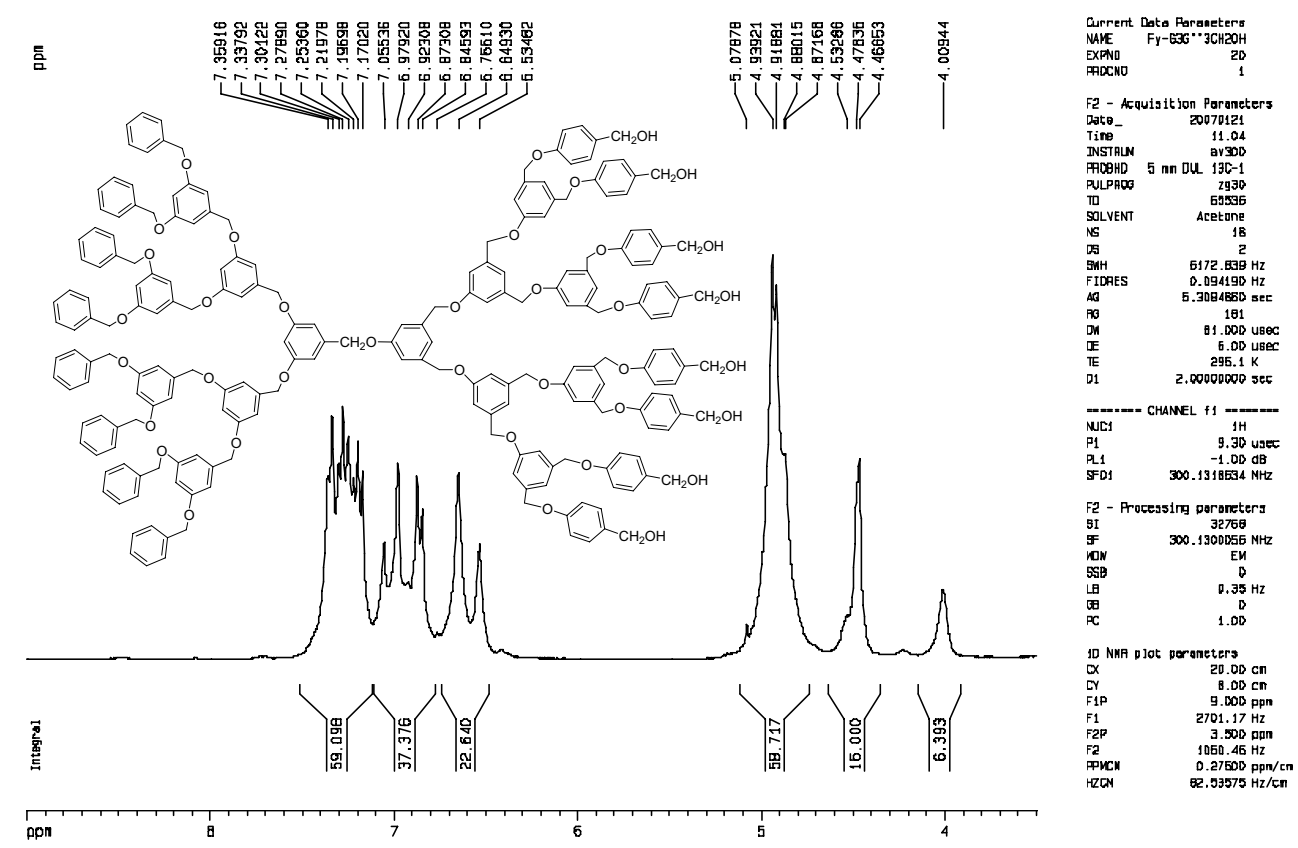

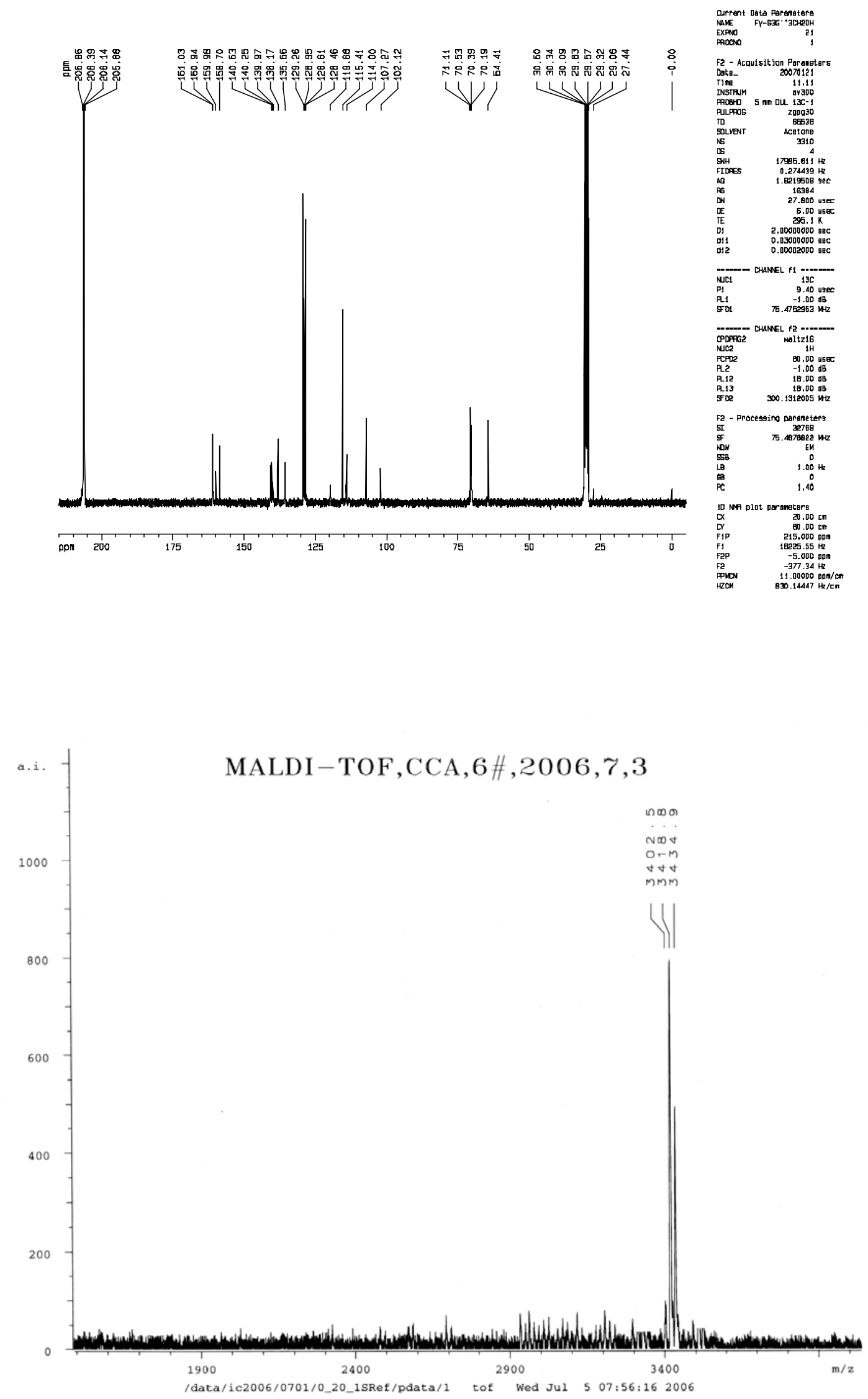


\section{5. ${ }^{1} \mathrm{H}$ and ${ }^{13} \mathrm{C}$ NMR and MS spectra of the dendritic Suzuki coupling reaction products}

Figure S10. ${ }^{1} \mathrm{H}$ NMR, ${ }^{13} \mathrm{C}$ NMR and MS spectra of Janus Dendrimers 3a
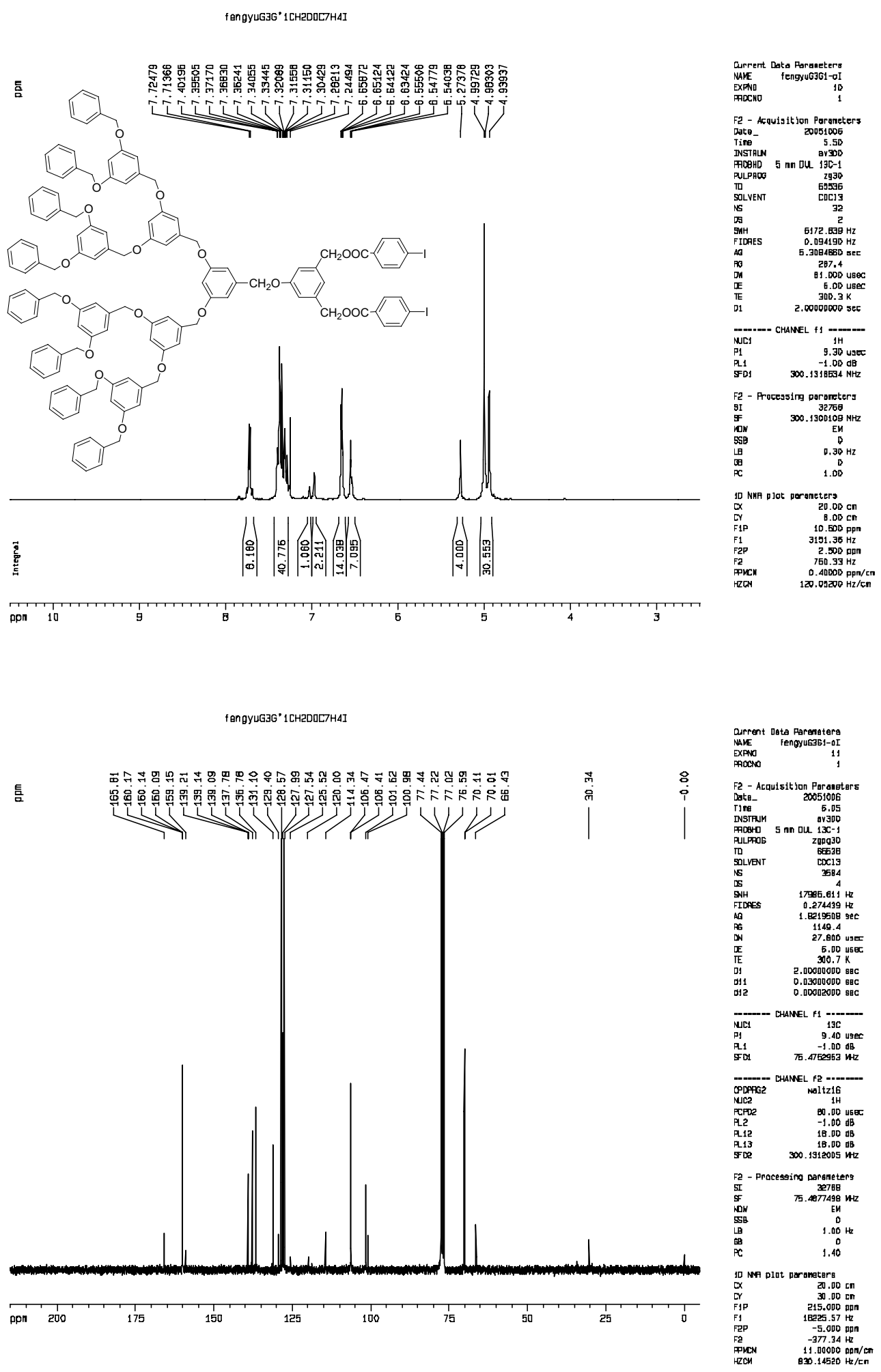


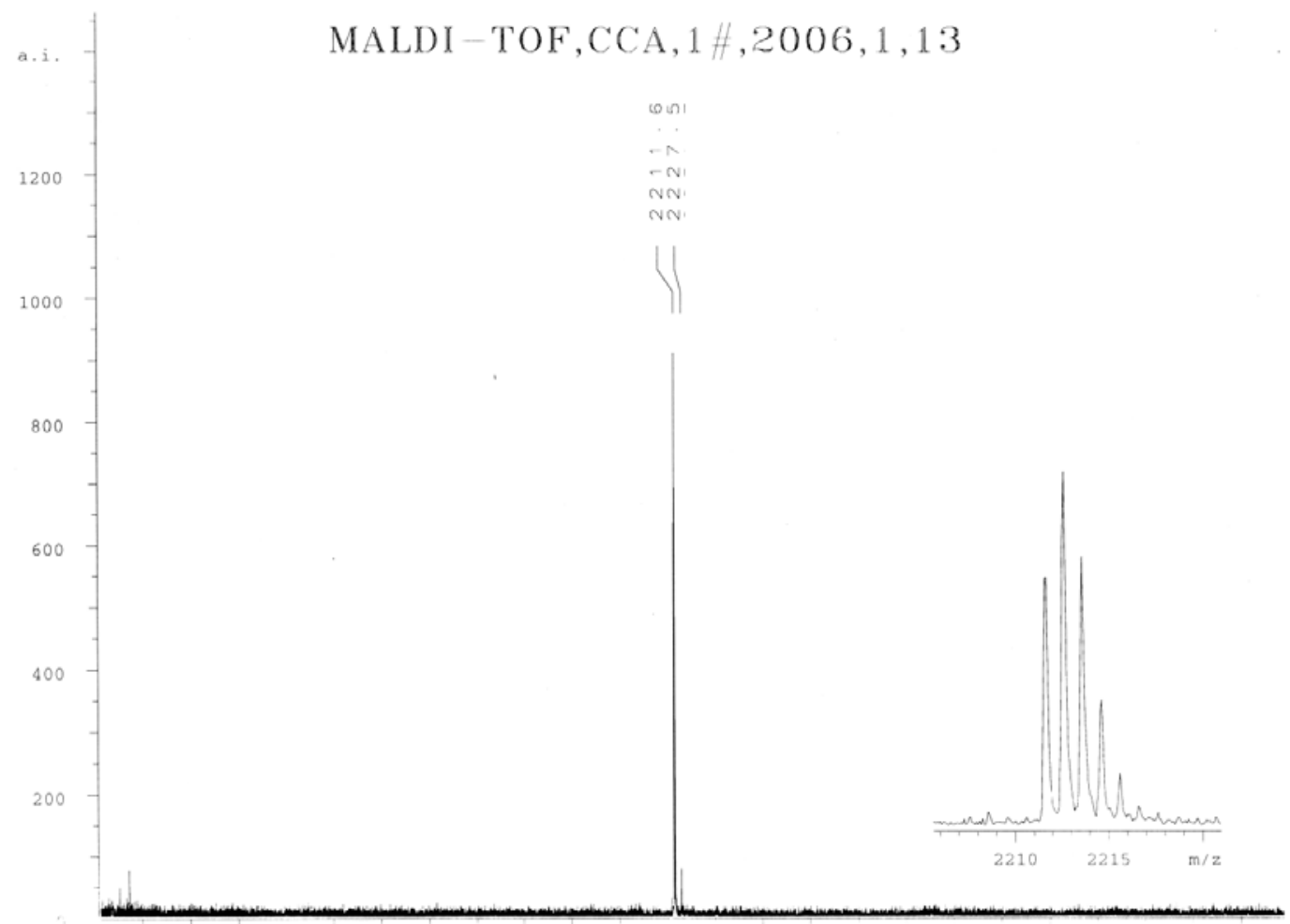

Figure S11. ${ }^{1} \mathrm{H}$ NMR, ${ }^{13} \mathrm{C}$ NMR and MS spectra of Janus Dendrimers $3 \mathbf{b}$
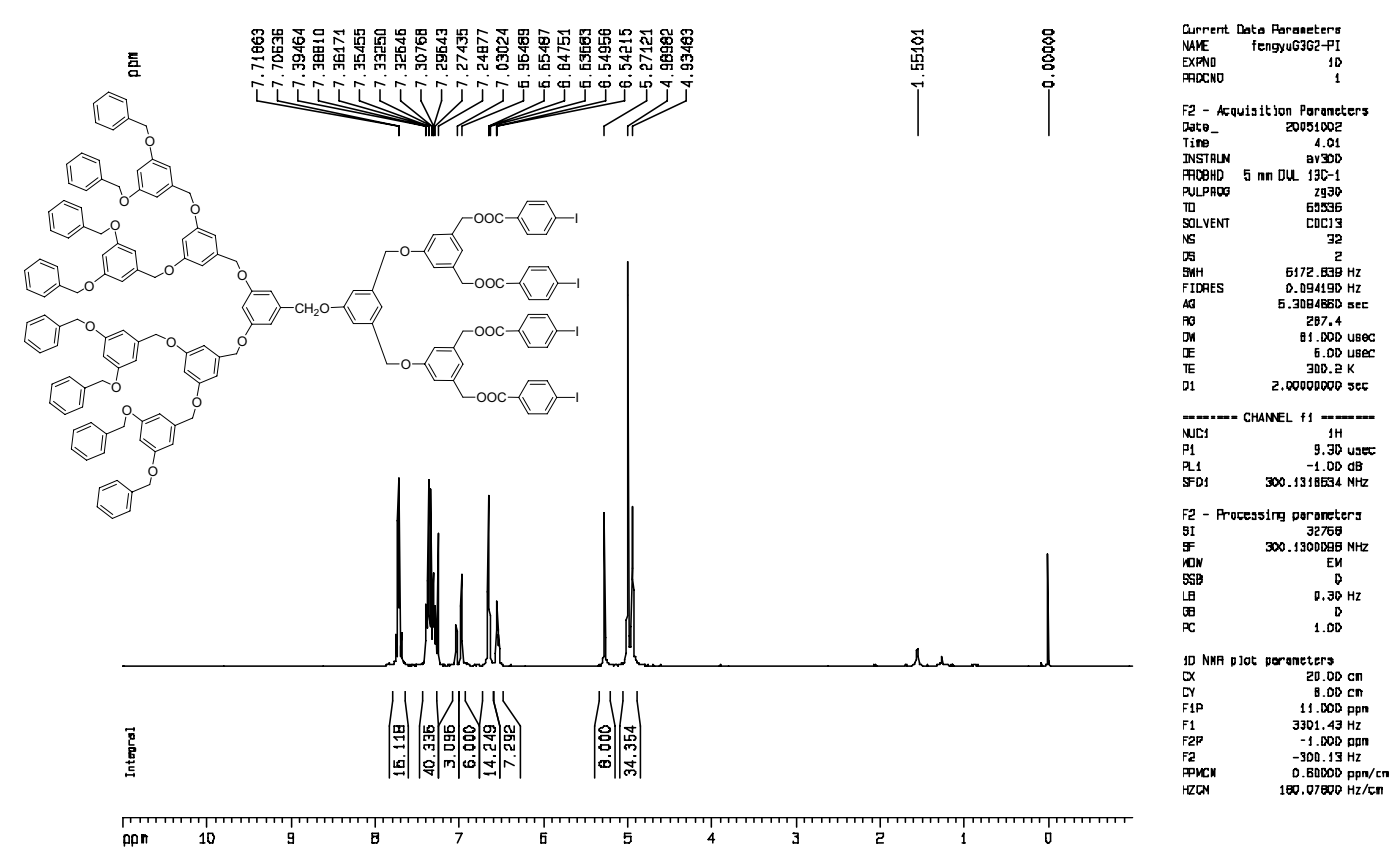


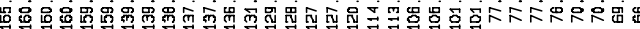

(

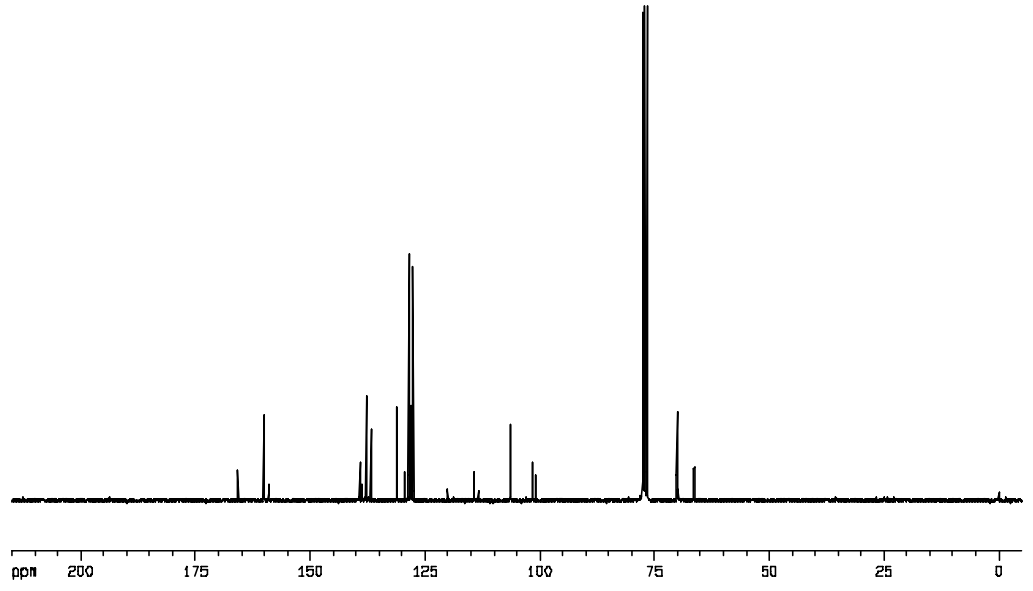

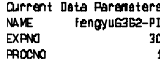

F2 - Acquisition Poranters

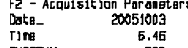

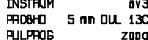

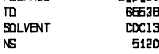

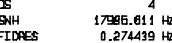

$1.8219500 \mathrm{sec}$

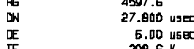

$0.000000 \mathrm{seC}$
011

NUC1 - CHANEL A1

$\begin{array}{ll}\text { PH } & 9.40 \text { uge } \\ \text { PL1 } & -1.00 \mathrm{~dB}\end{array}$

CPDOAGL2 CHANEL Re -.......

$\begin{array}{ll}\text { PCPOE } & 80.00 \mathrm{usec} \\ \text { PL2 } & -1.00 \mathrm{~dB}\end{array}$

$\begin{array}{lr}\text { PL12 } & 18.00 \mathrm{~dB} \\ \text { PL13 } & 18.00 \mathrm{~dB} \\ \text { STCR } & 300.1912005 \mathrm{WHB}\end{array}$

Fe - Processing paraneters
gI
32768

$\begin{array}{lc}\text { SI } & 32768 \\ \text { SF } & 75.4877499 \mathrm{HE} \\ \text { NOW } & \text { EM } \\ \text { SSB } & 0 \\ \text { LB } & 1.00 \mathrm{HE} \\ \text { GB } & 0 \\ \text { PC } & 1.40\end{array}$

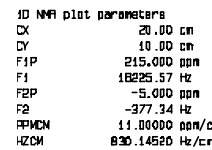

MALDI TOF,COA, $2006,1,33$

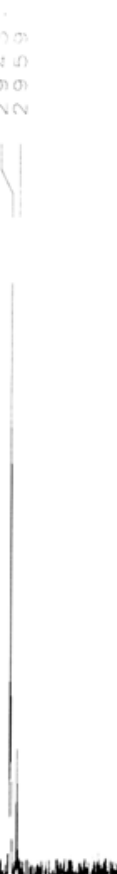


Figure S12. ${ }^{1} \mathrm{H}$ NMR, ${ }^{13} \mathrm{C}$ NMR and MS spectra of Janus Dendrimers $3 \mathrm{c}$
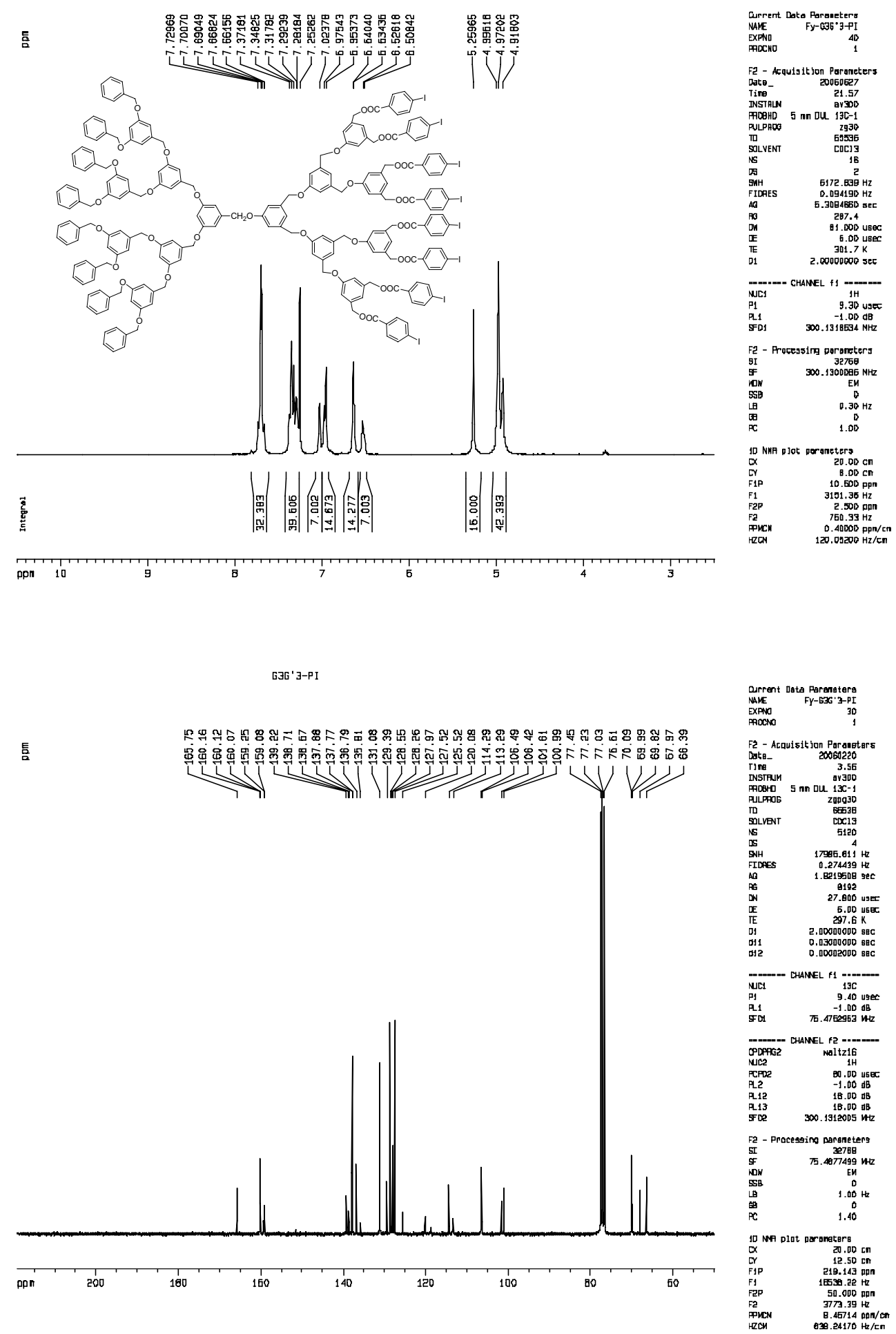


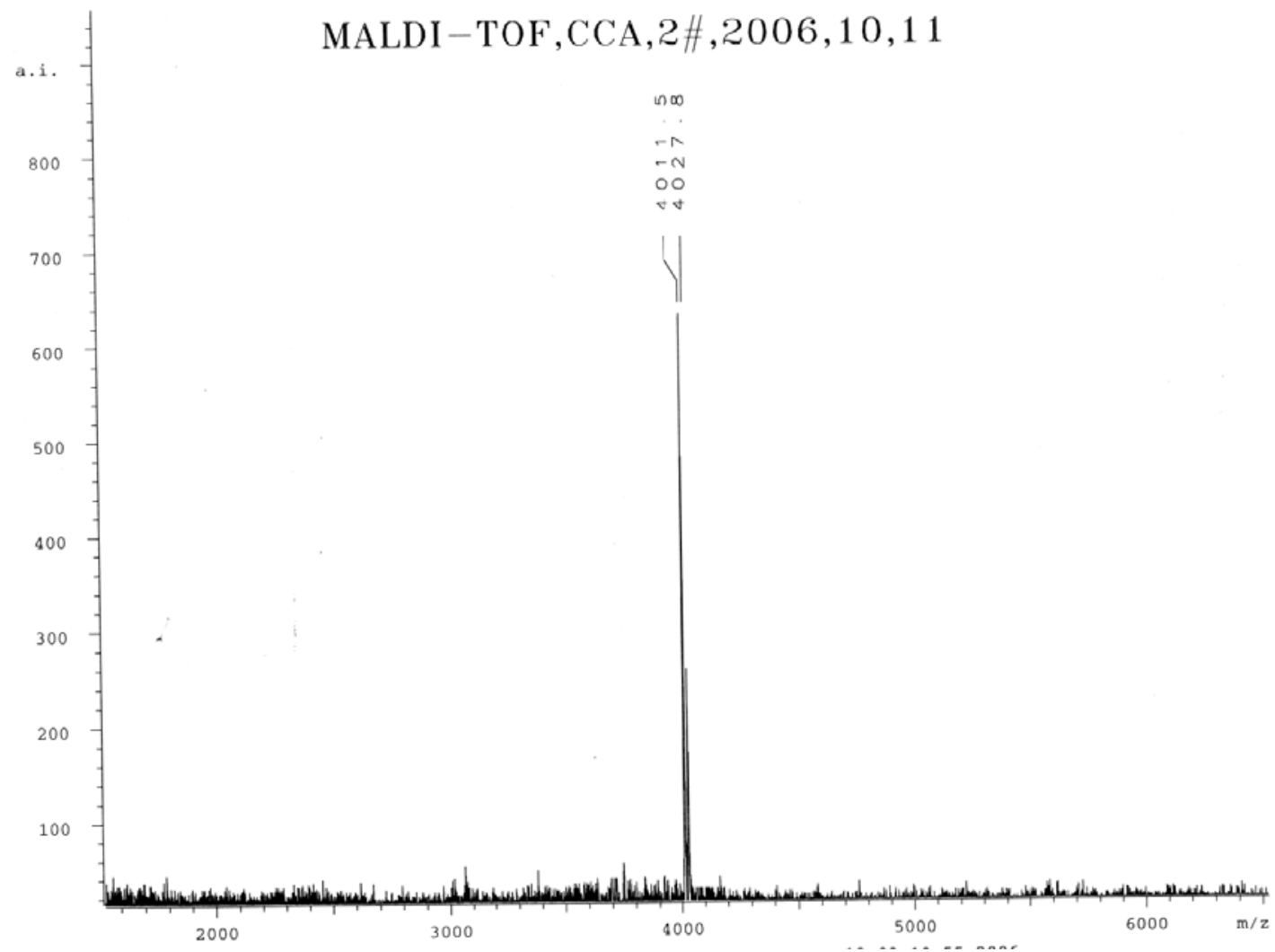

Figure S13. ${ }^{1} \mathrm{H}$ NMR, ${ }^{13} \mathrm{C}$ NMR and MS spectra of Janus Dendrimers 3d
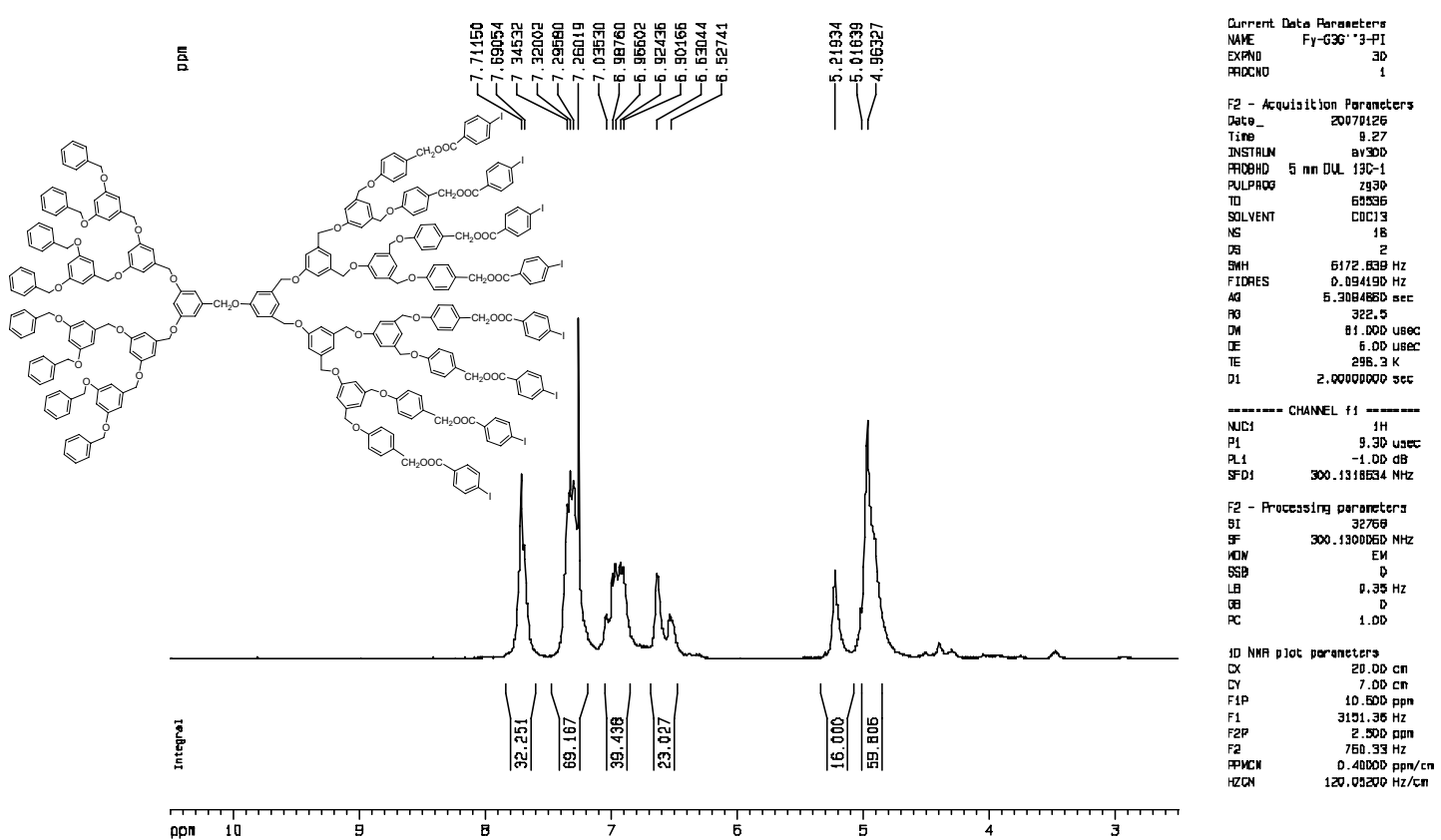


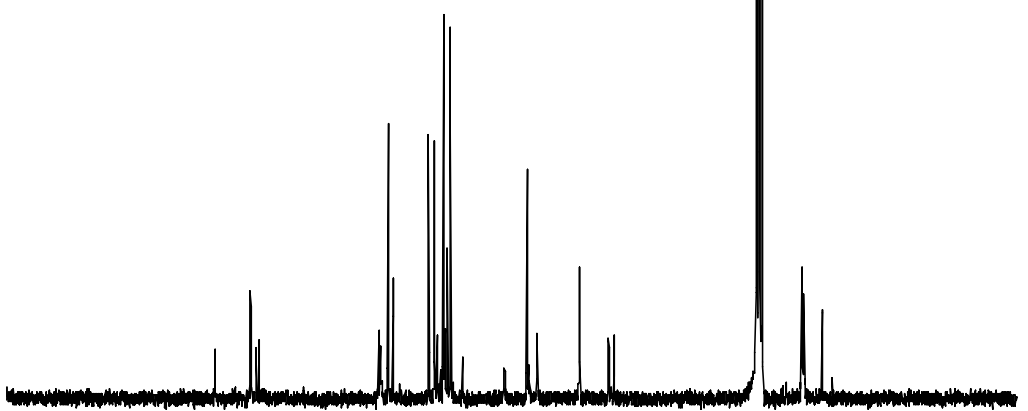

NUC1 - CHANEL A1

$\begin{array}{lr}\text { PS } & 9.40 \text { ugec } \\ \text { PL1 } & -1.00 \text { dB } \\ \text { SFO1 } & \pi .4752963 \mathrm{WH}\end{array}$

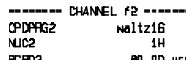

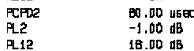

$\begin{array}{lr}\text { PL12 } & 18.00 \mathrm{~dB} \\ \text { PLL13 } & 18.00 \mathrm{~dB} \\ \text { FFC } & 300.1912005 \mathrm{WHz}\end{array}$

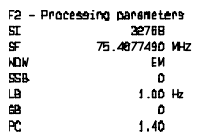
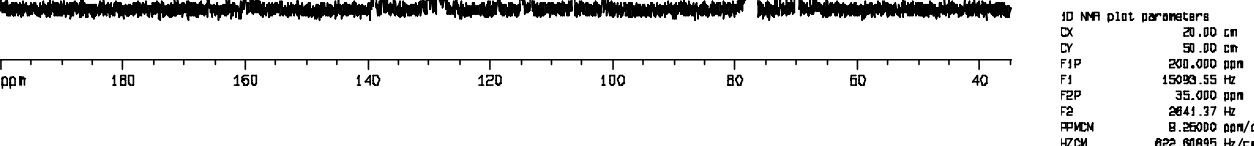

MALDI TOF,CCA,G:3G:3" PI,2006, 12,29

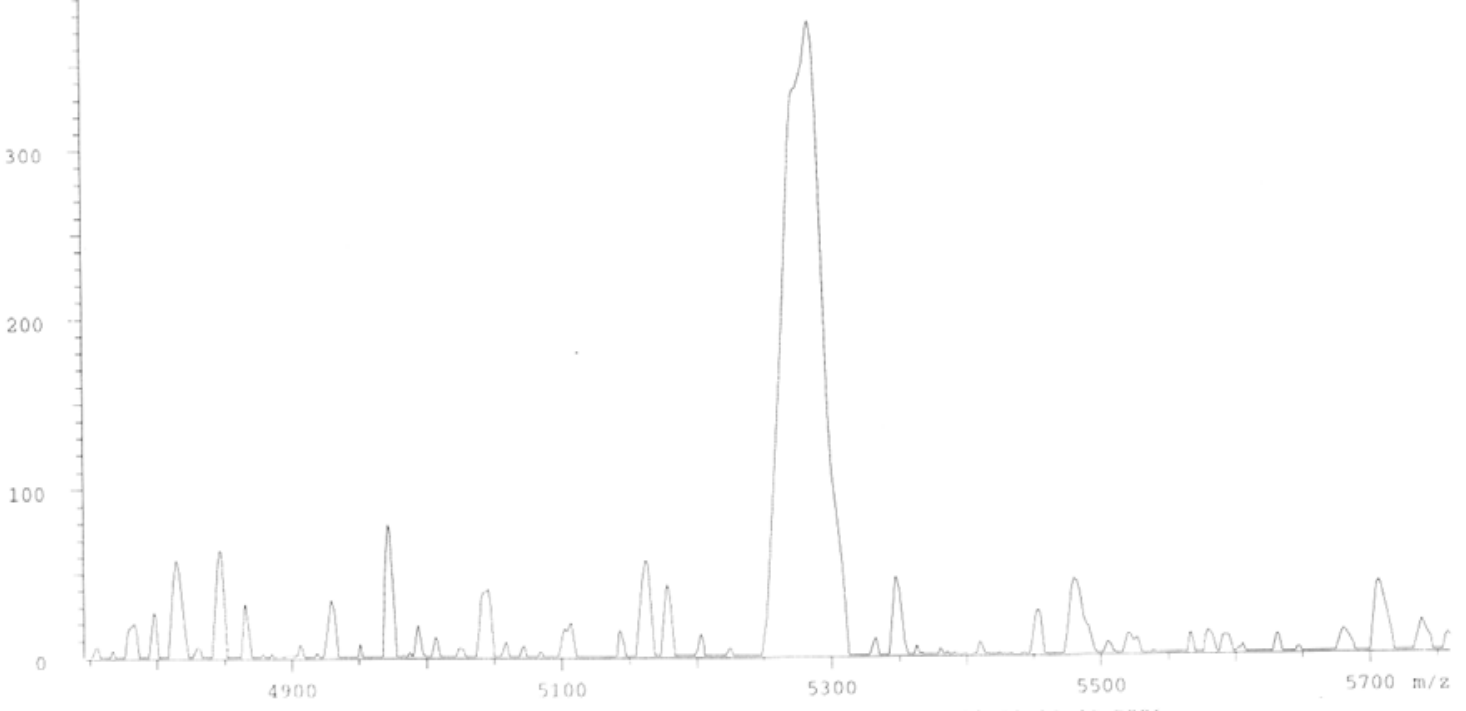


Figure S14. ${ }^{1} \mathrm{H}$ NMR, ${ }^{13} \mathrm{C}$ NMR and MS spectra of Janus Dendrimers 4aa
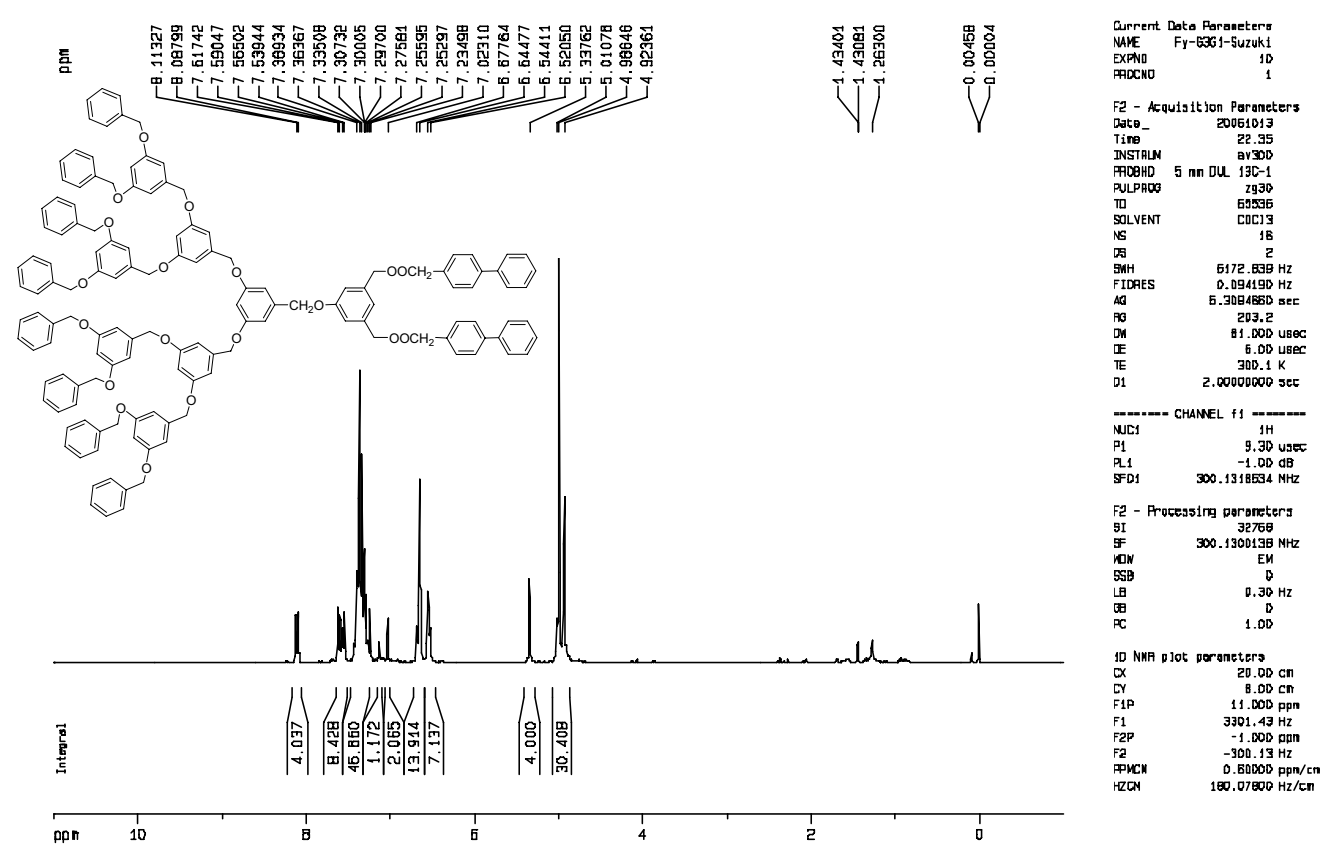

GJG'1-Suzuki Reaction
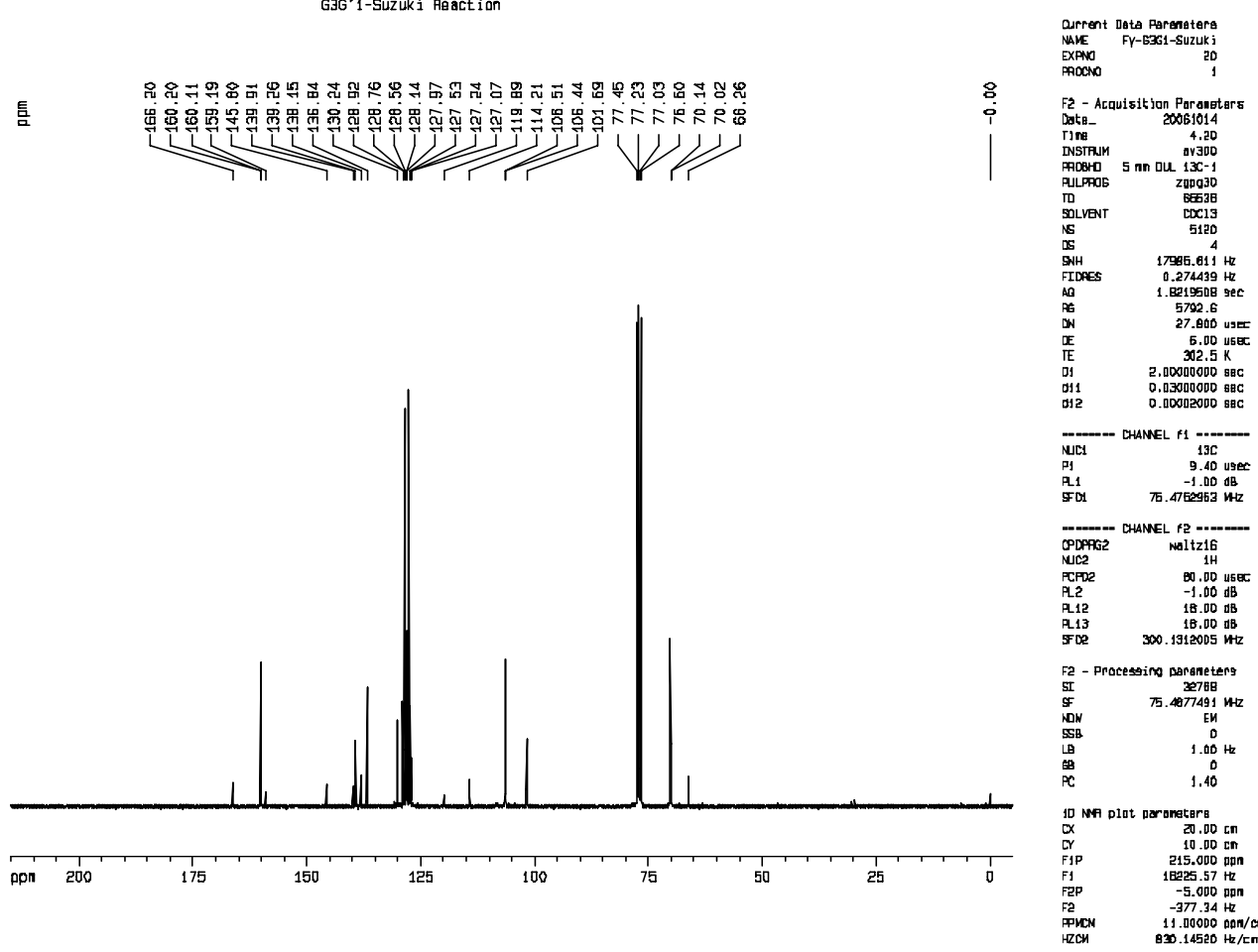


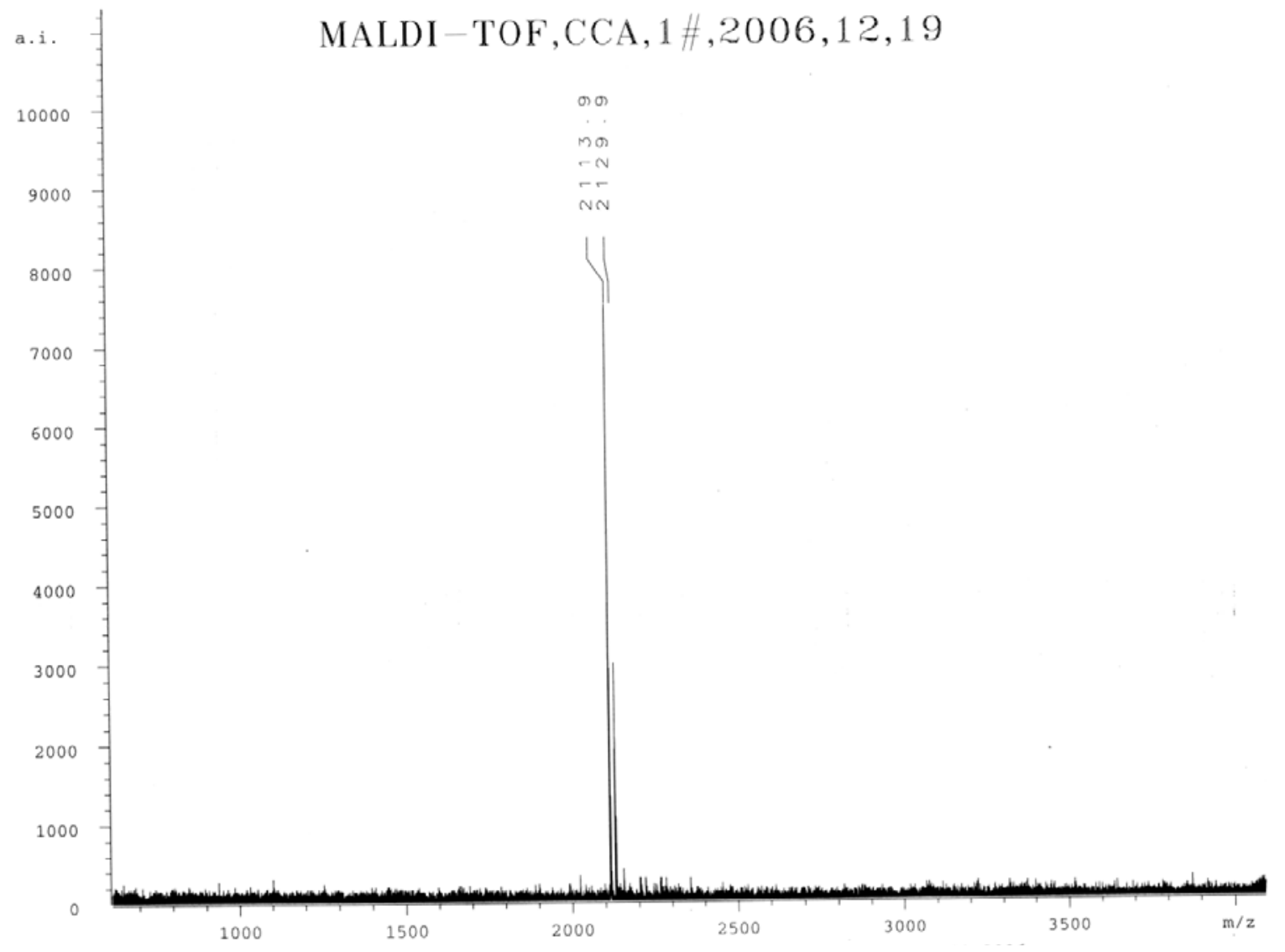

Figure S15. ${ }^{1} \mathrm{H}$ NMR, ${ }^{13} \mathrm{C}$ NMR and MS spectra of Janus Dendrimers 4 ba
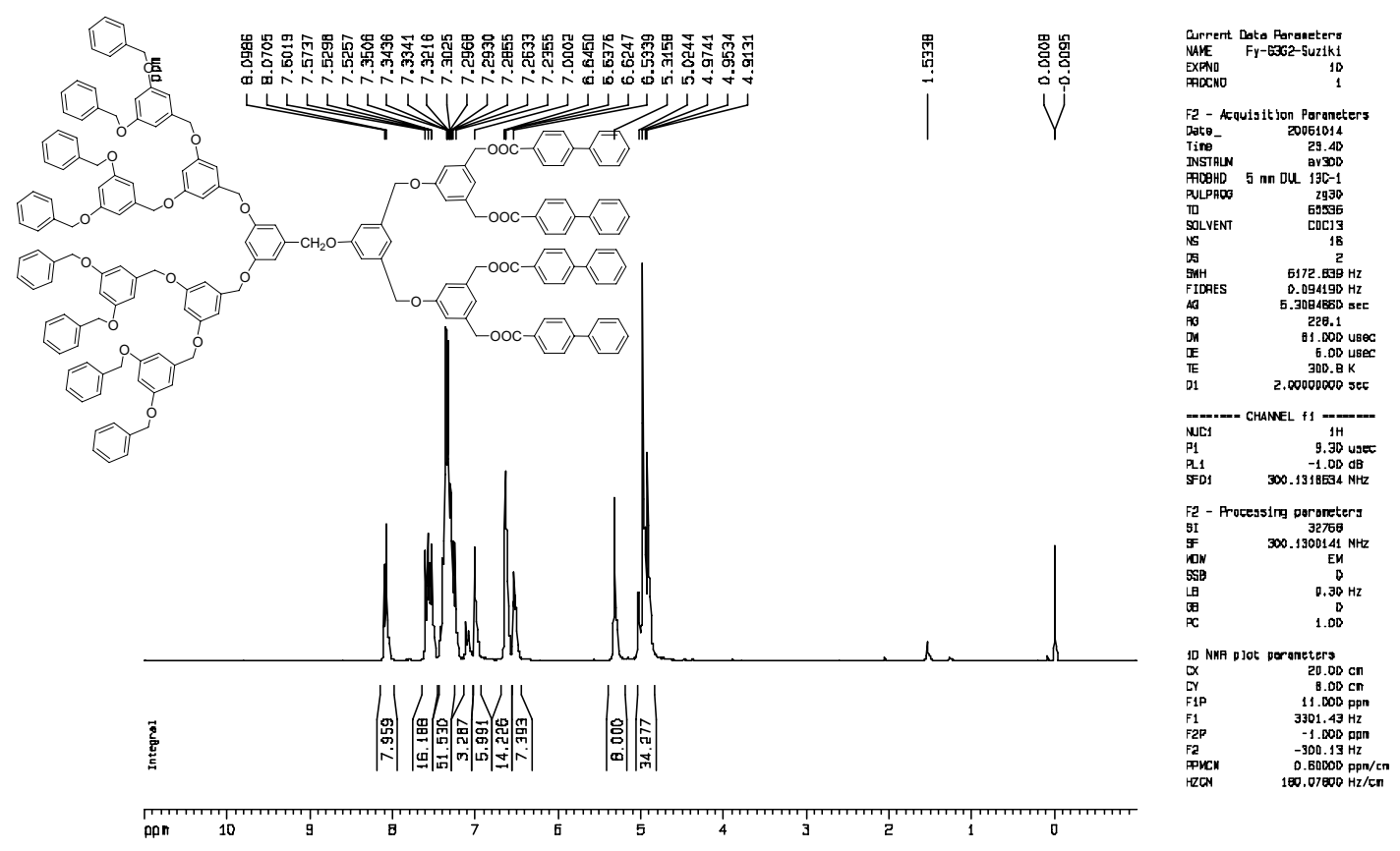


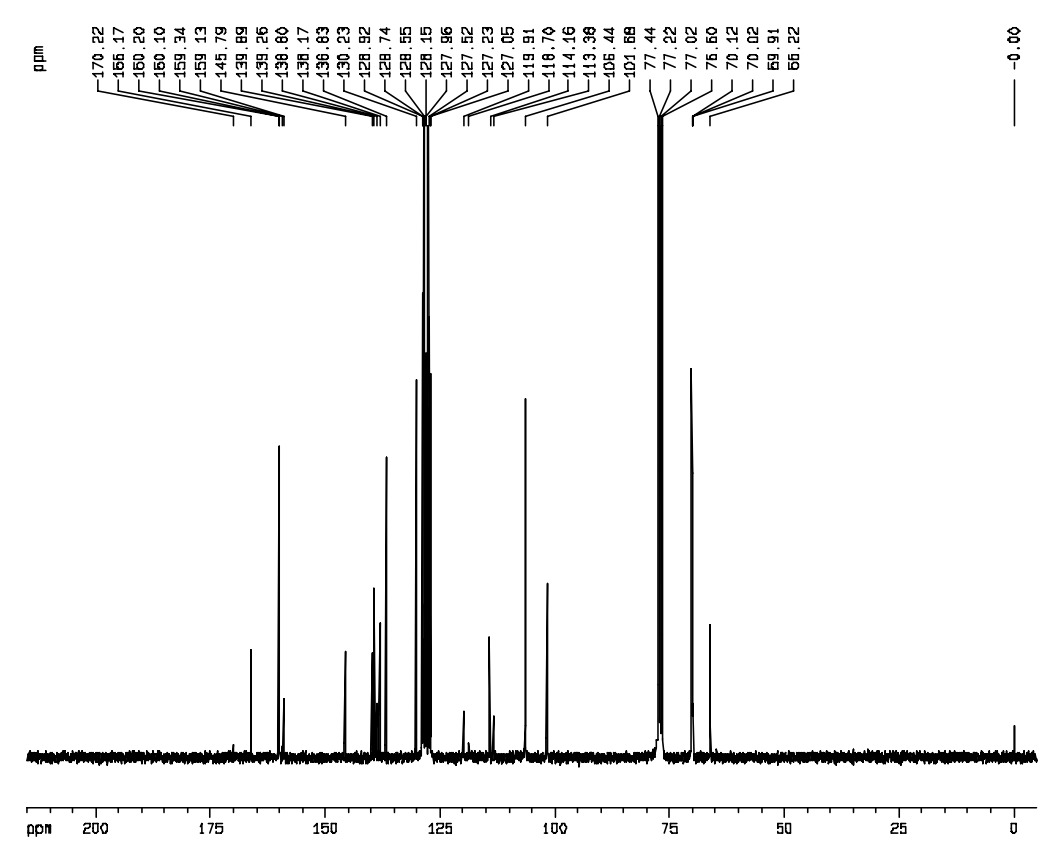

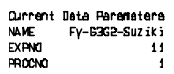

F2 - Acquisition Poraneters

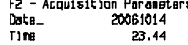

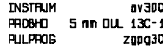

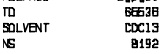

$\begin{array}{ll}\text { DS } & 17586.611 \\ \text { SNH } & \text { Hz } \\ \text { FIDEES } & 0.274439 \mathrm{HZ}\end{array}$

$\begin{array}{ll}\text { AO } & 1.821950 \mathrm{gec} \\ \mathrm{BG} & 5160 \mathrm{ge}\end{array}$

$\begin{array}{ll}\mathrm{DN} & 27.000 \text { user } \\ \mathrm{DE} & 5.00 \mathrm{usec}\end{array}$

$\begin{array}{ll}0 . & 2.00000000 \mathrm{seC} \\ 01 & 0.03000000 \mathrm{seC}\end{array}$

d. 0.00002000880

NUC1 - CHANEL F1 -...-

$\begin{array}{lr}\text { PY } & 9.40 \text { ugec } \\ \text { PL1 } & -1.00 \mathrm{~dB} \\ \text { SFO1 } & \pi .4752963 \mathrm{WE}\end{array}$

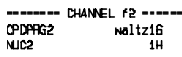

$\begin{array}{ll}\text { PCPO2 } & 80.000580 \\ \text { PL2 } & -1.00 \mathrm{~dB}\end{array}$

$\begin{array}{lr}\text { PL12 } & 18.00 \mathrm{~dB} \\ \text { PLL13 } & 18.00 \mathrm{~dB} \\ \text { FFC } & 300.1912005 \mathrm{WHz}\end{array}$

Fe - Processing paraneters
gI

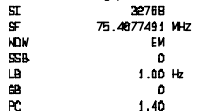

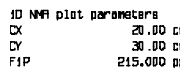

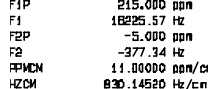

MALDI TOF,CCA,2\#,2006,12,19 
Figure S16. ${ }^{1} \mathrm{H}$ NMR, ${ }^{13} \mathrm{C}$ NMR and MS spectra of Janus Dendrimers 4 ca
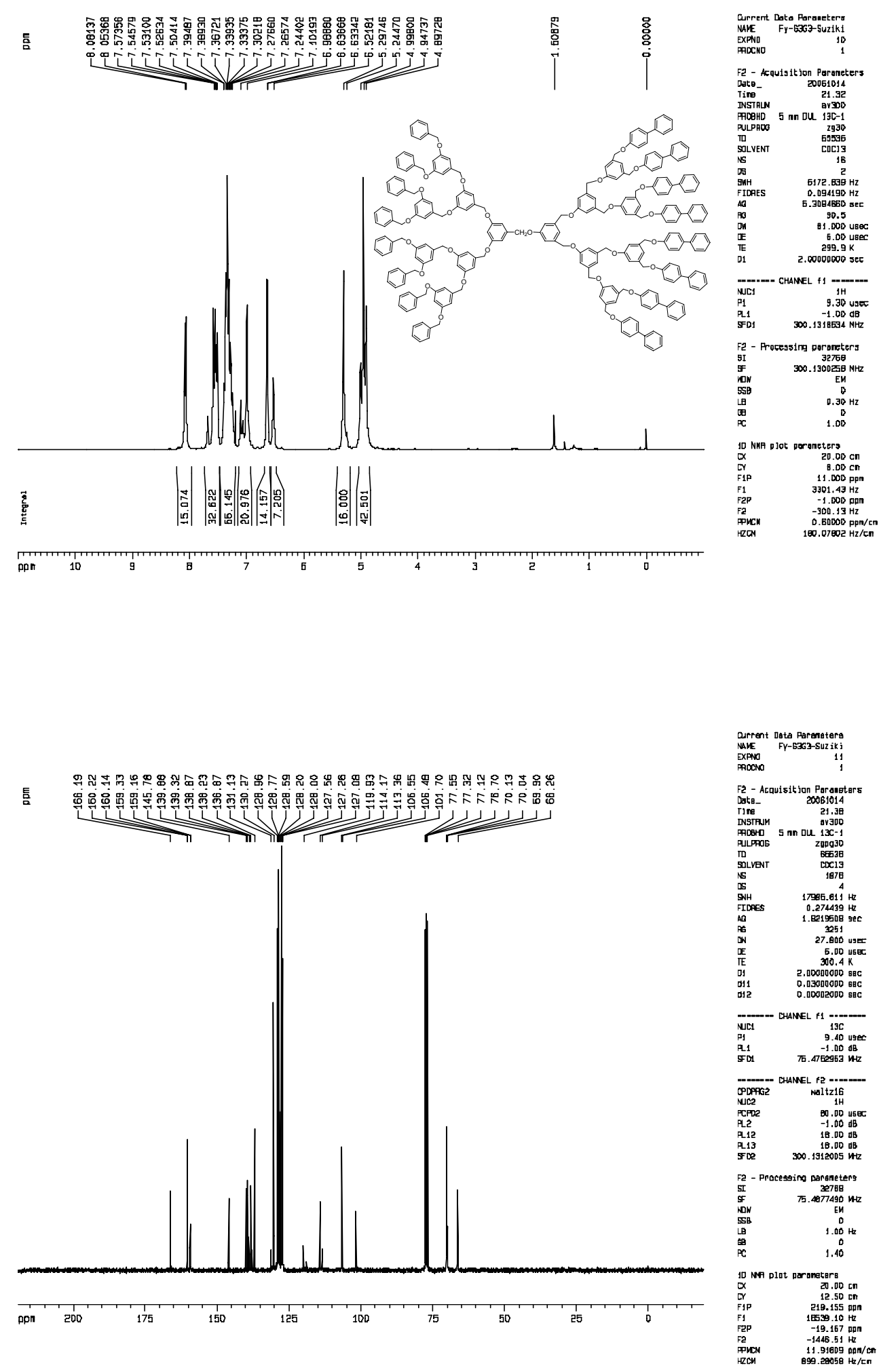


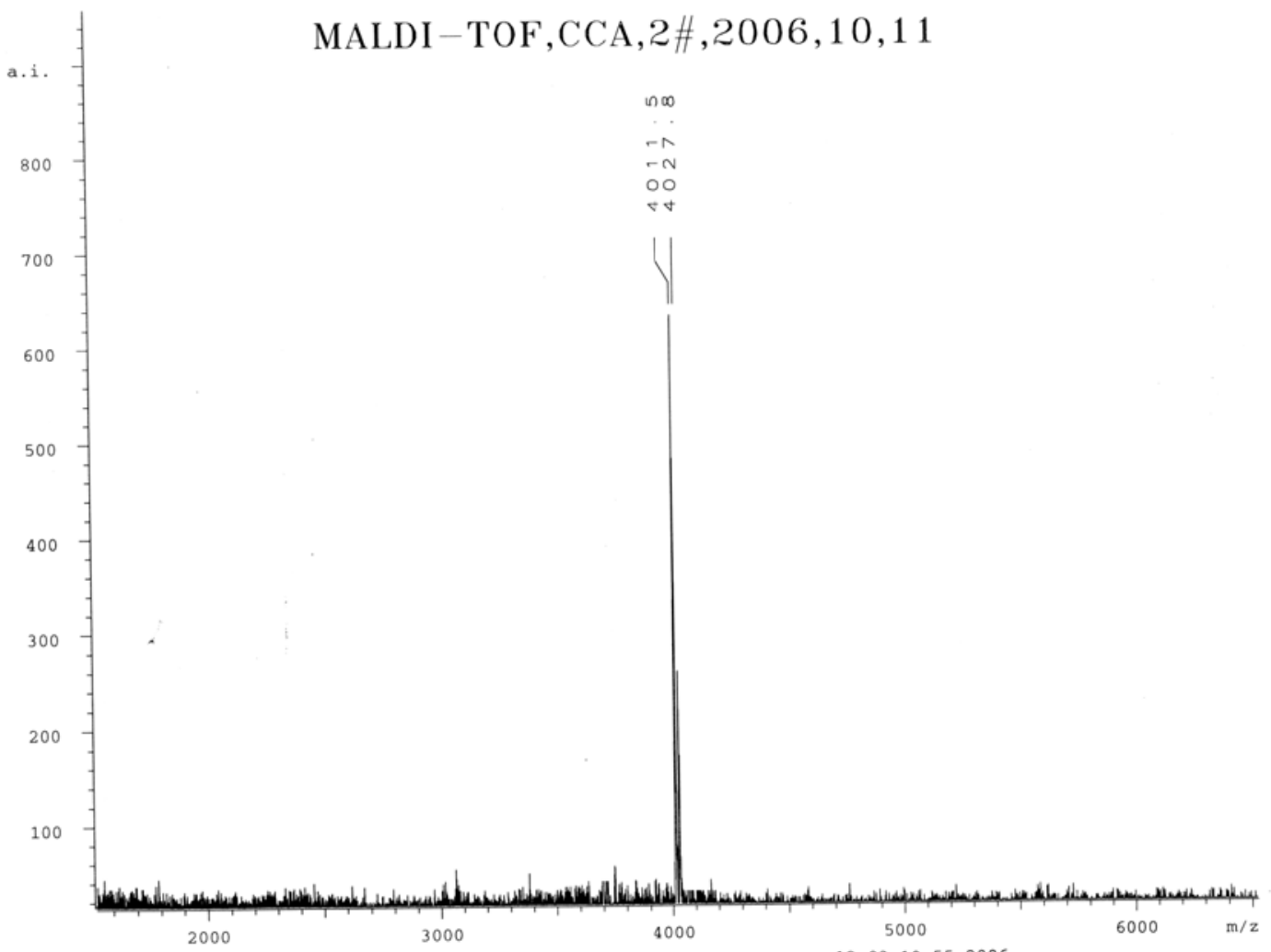

Figure S17. ${ }^{1} \mathrm{H}$ NMR, ${ }^{13} \mathrm{C}$ NMR and MS spectra of Janus Dendrimers 4da

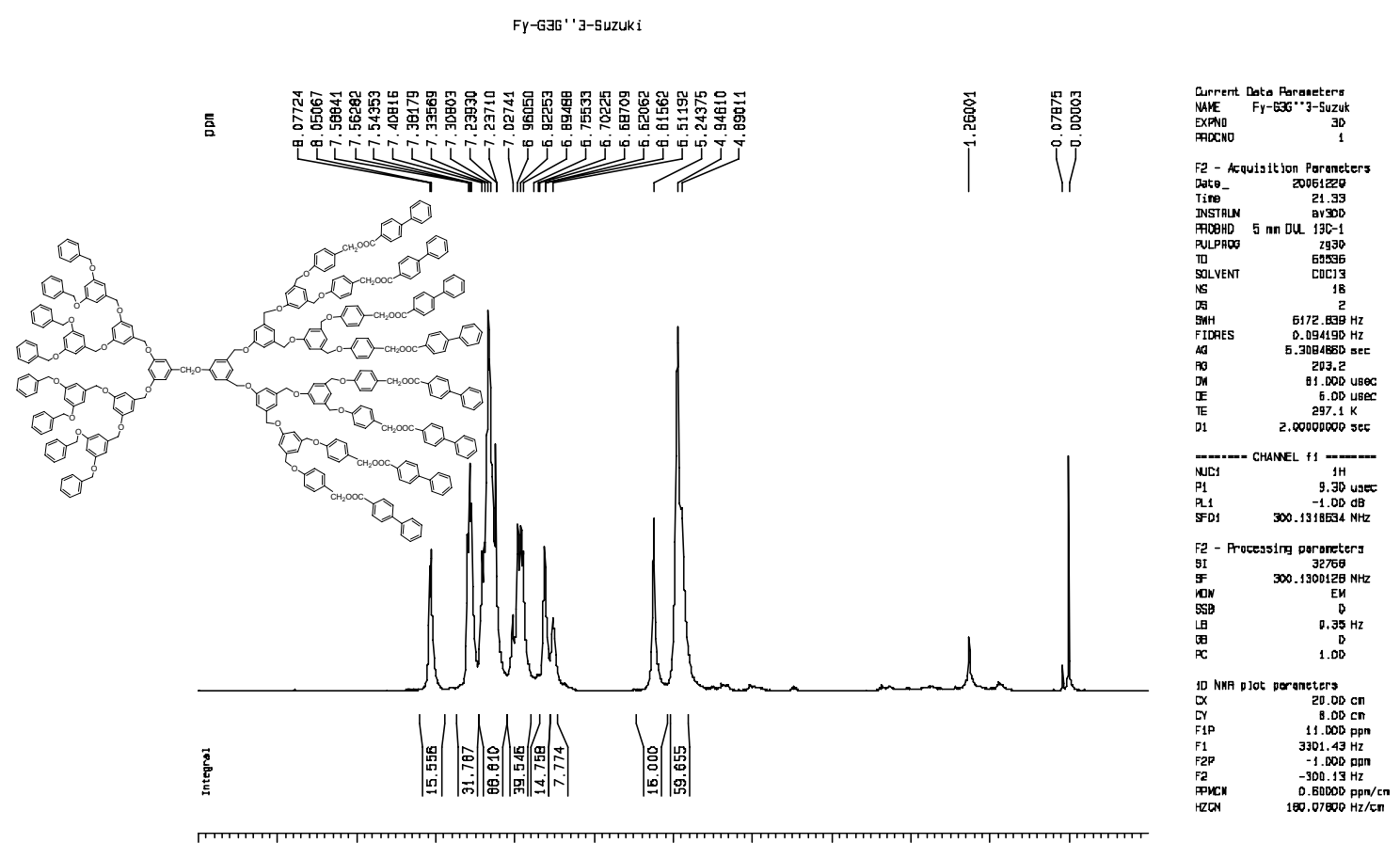



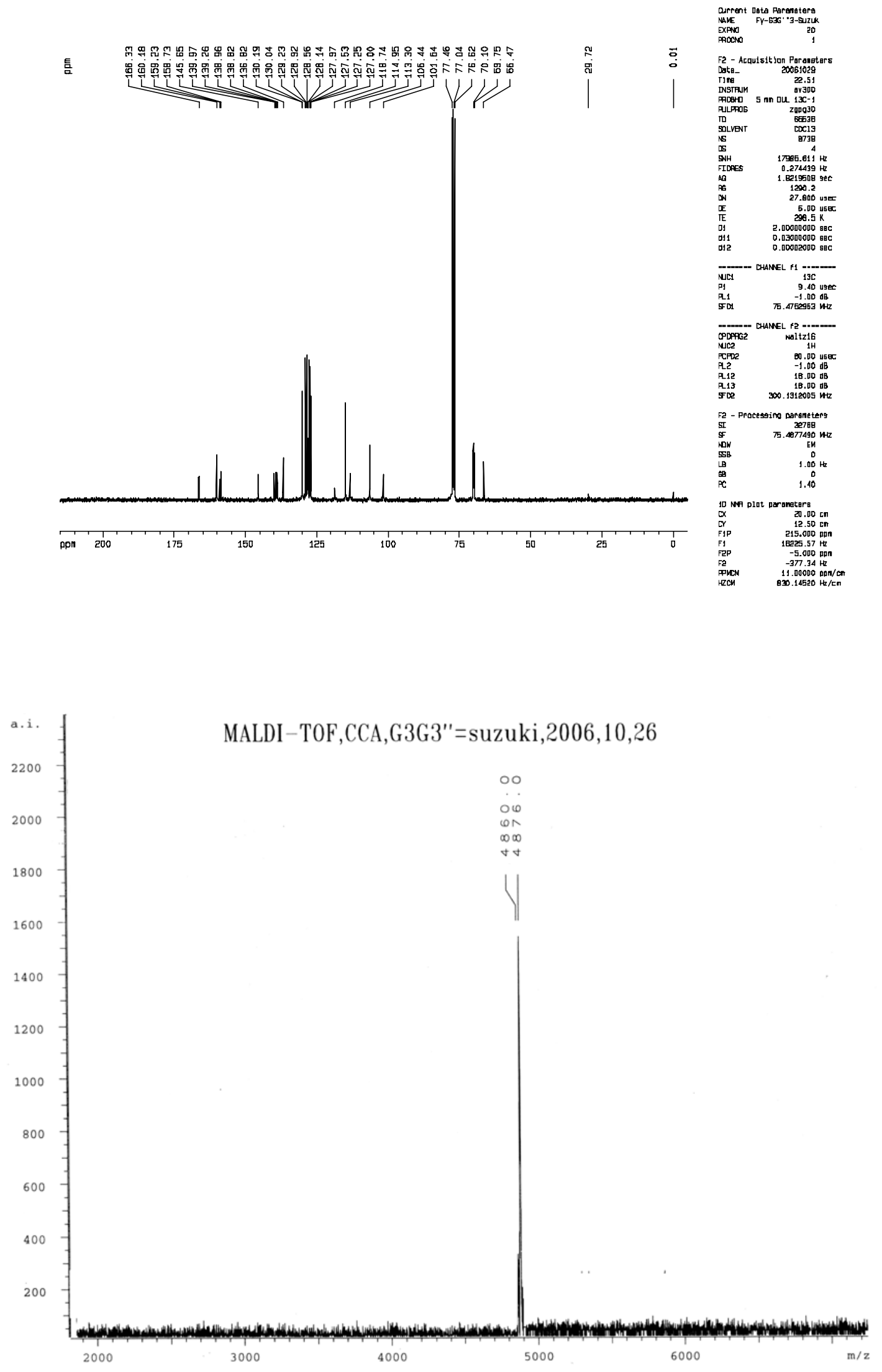
Figure S18. ${ }^{1} \mathrm{H}$ NMR, ${ }^{13} \mathrm{C}$ NMR and MS spectra of Janus Dendrimers 4cb
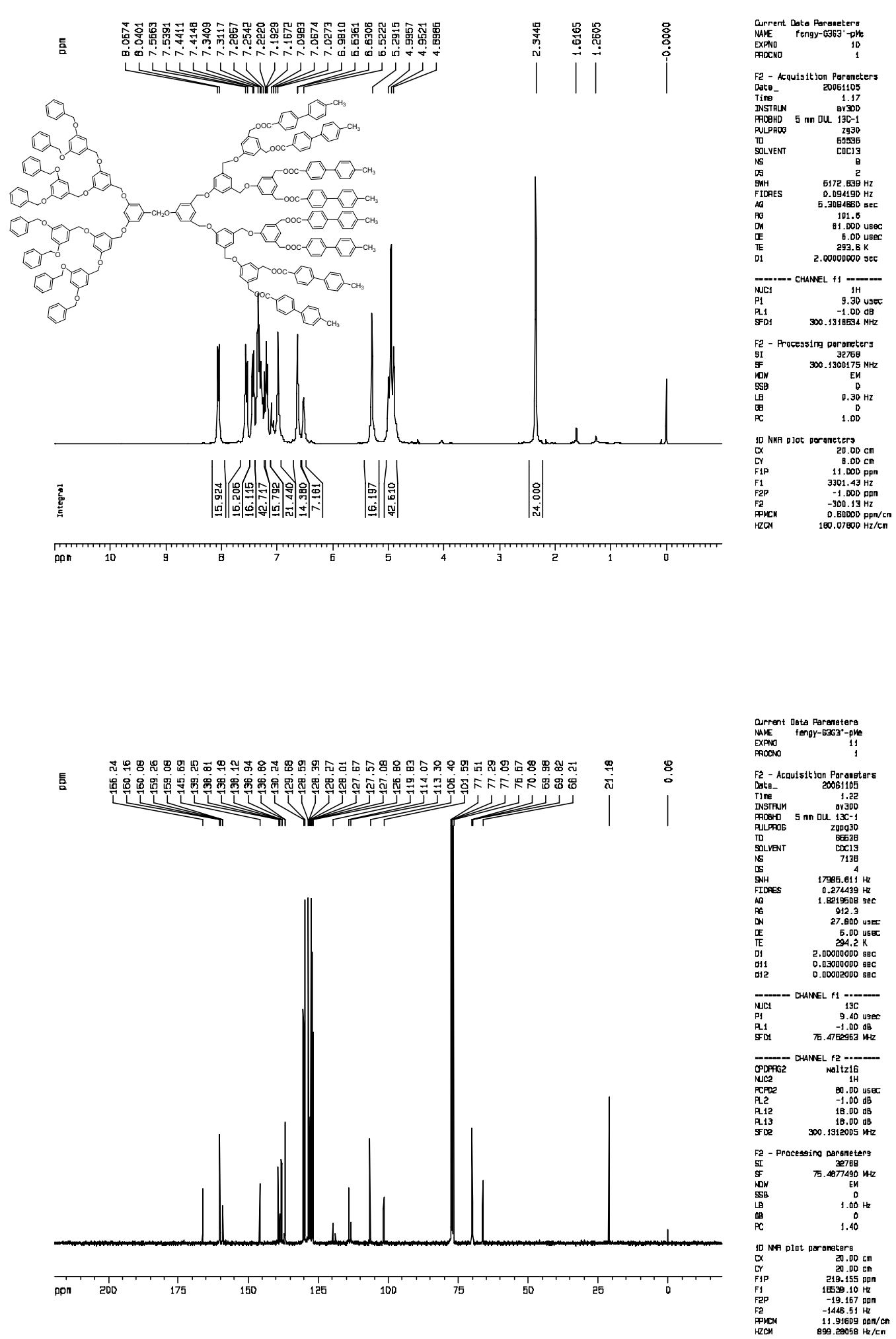


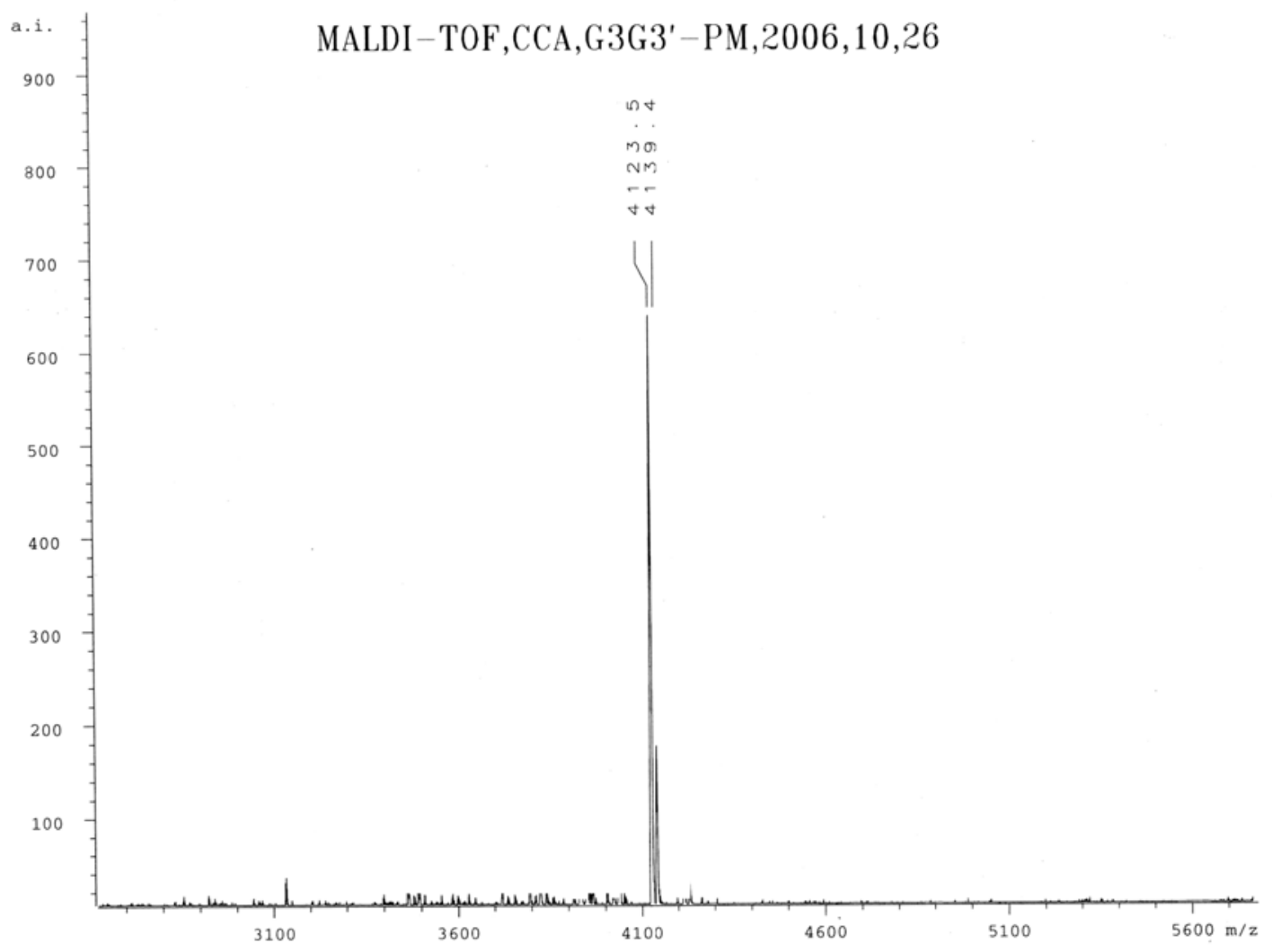

Figure S19. ${ }^{1} \mathrm{H}$ NMR, ${ }^{13} \mathrm{C}$ NMR and MS spectra of Janus Dendrimers 4ce

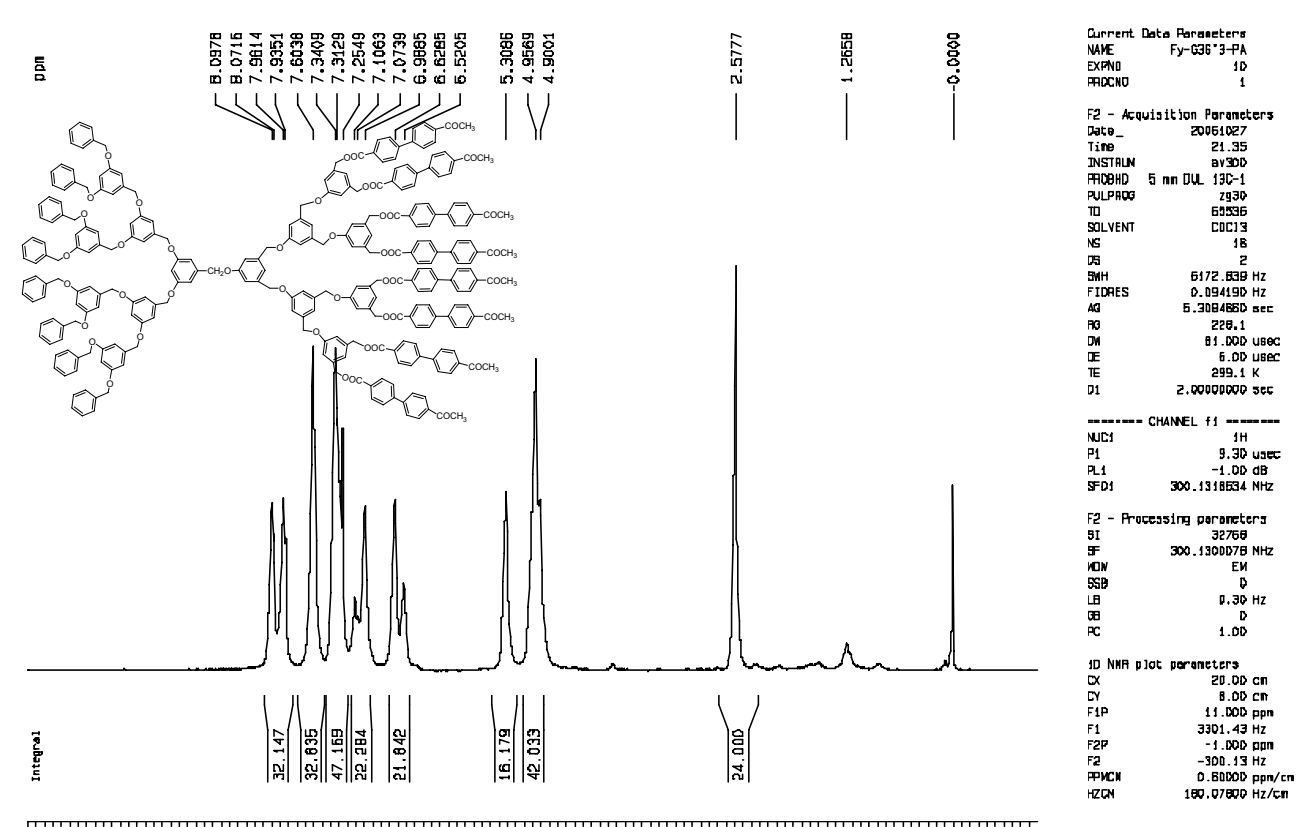



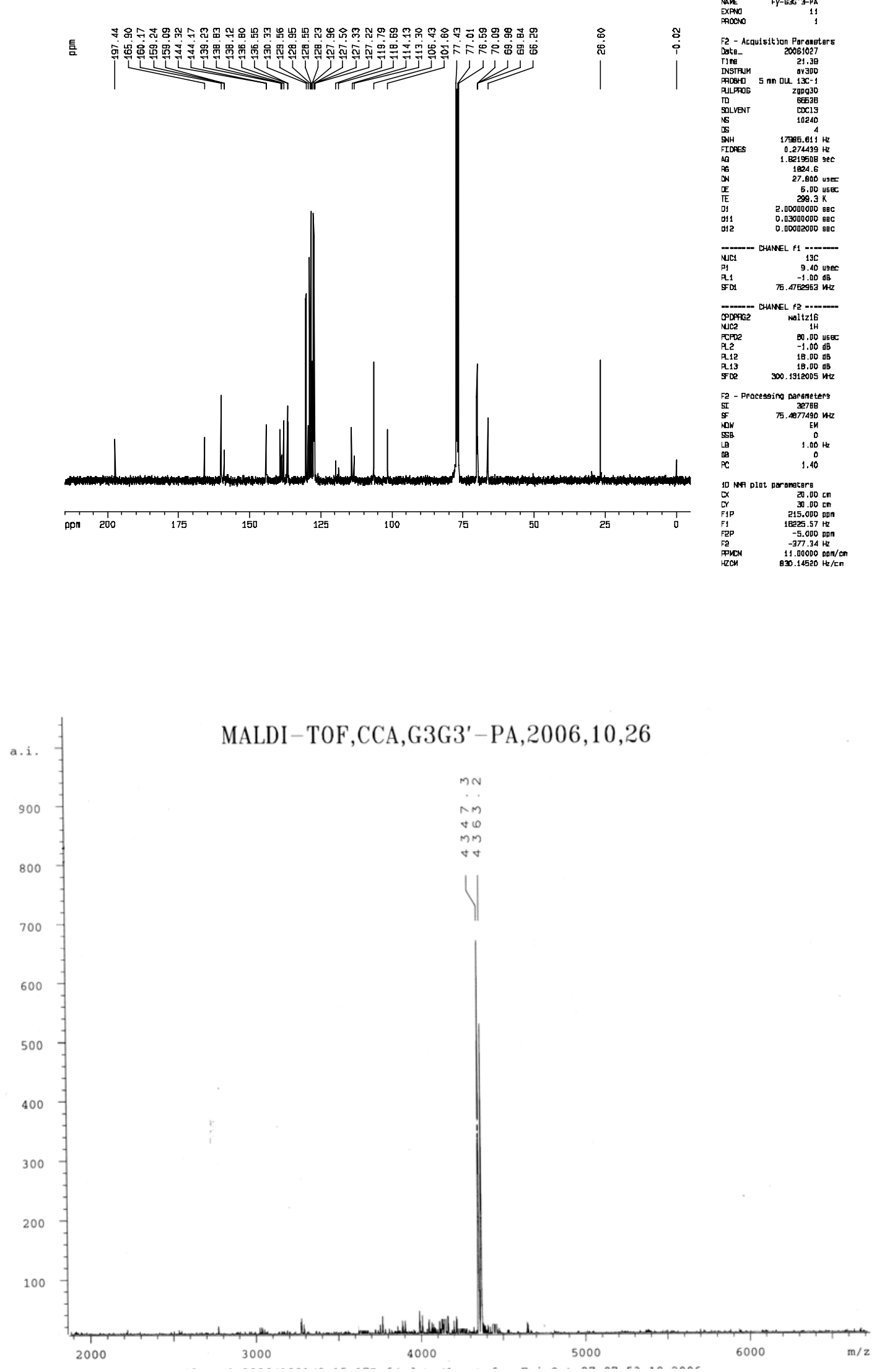
Figure S20. ${ }^{1} \mathrm{H}$ NMR, ${ }^{13} \mathrm{C}$ NMR and MS spectra of Janus Dendrimers 4cd
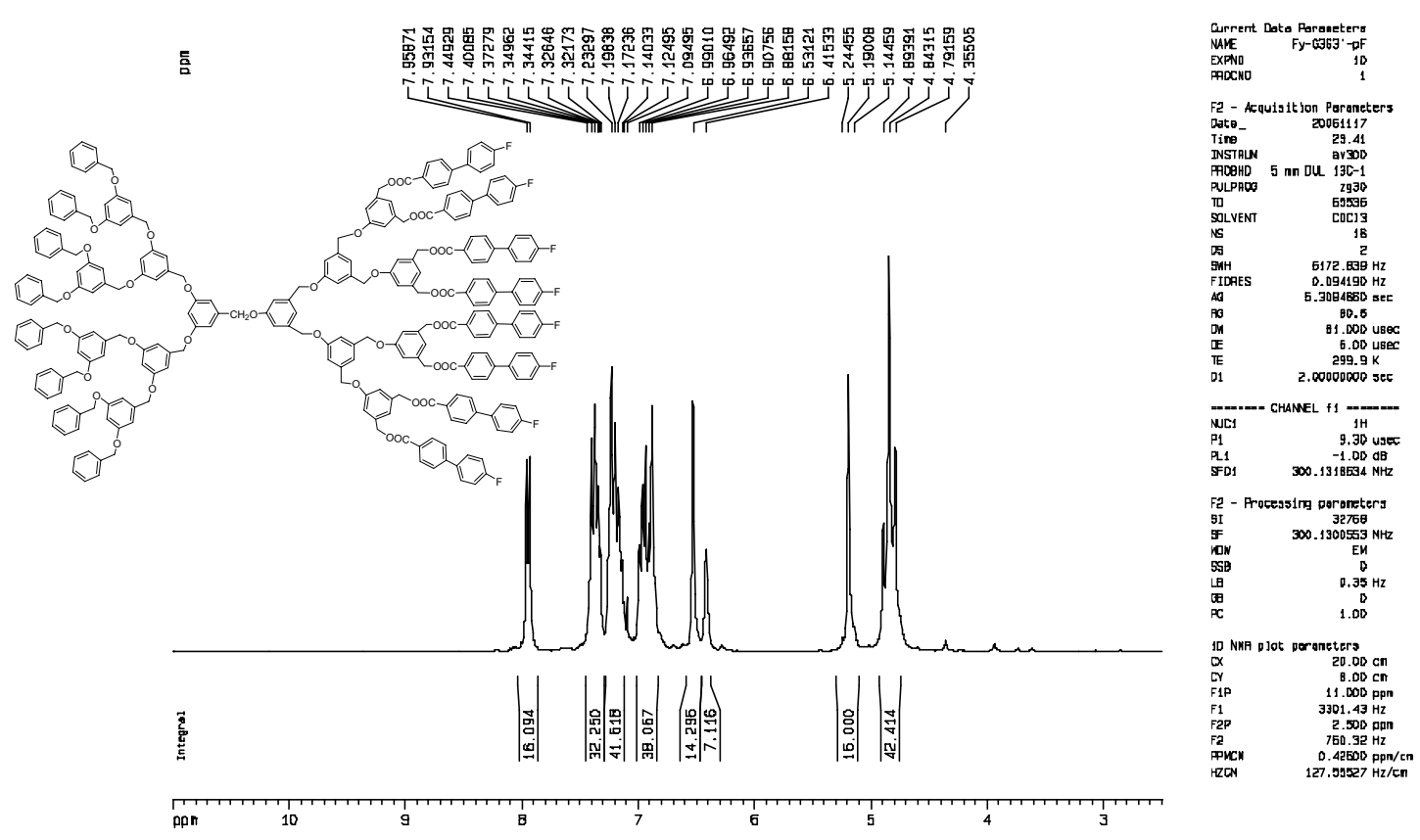

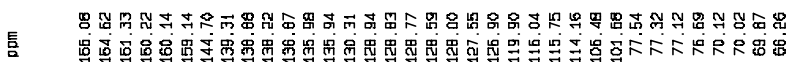
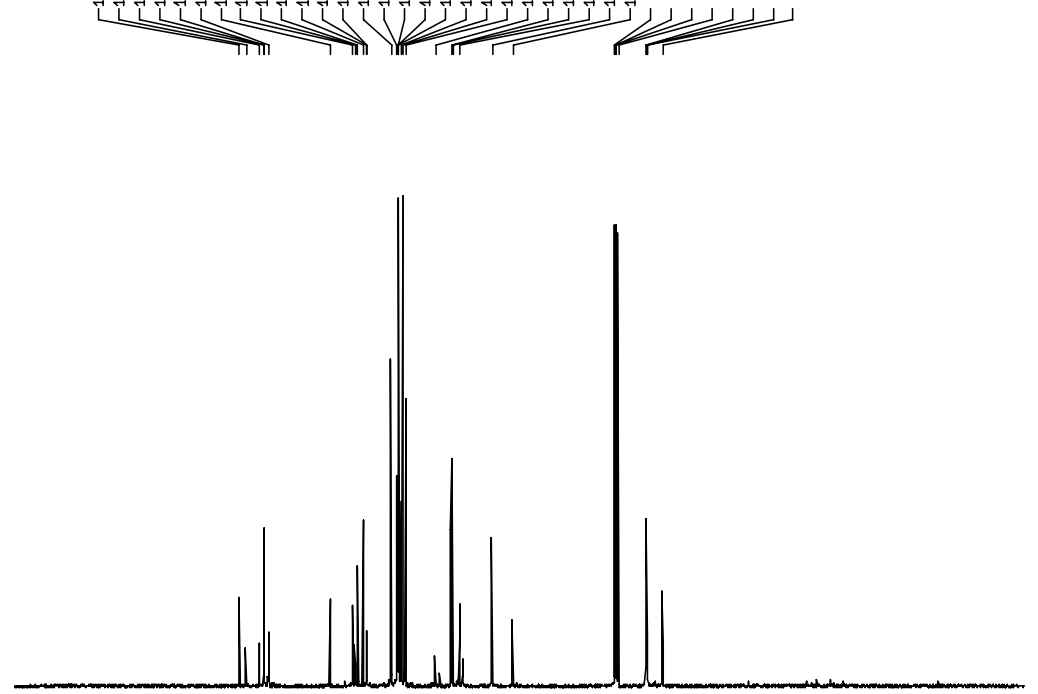

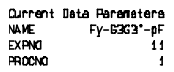

F2 - Acquisition Paranters
Data- 20061118

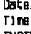

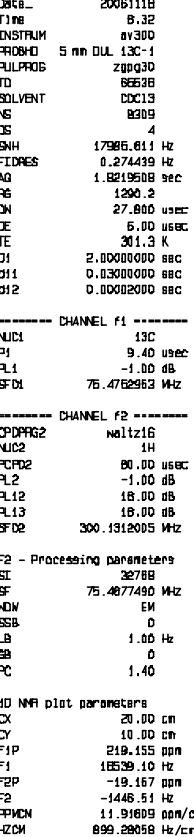




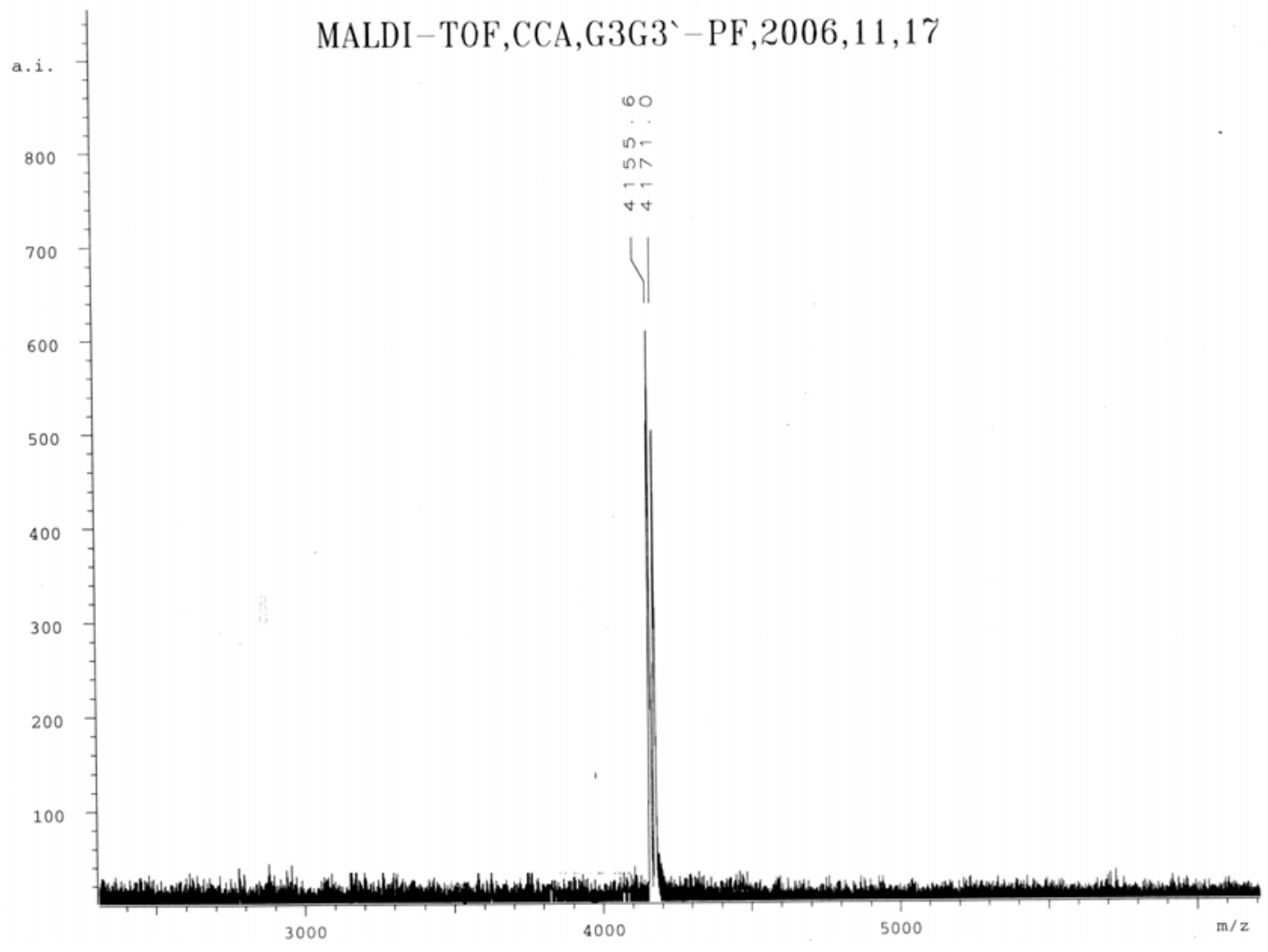




\section{6. ${ }^{1} \mathrm{H}$ and ${ }^{13} \mathrm{C}$ NMR spectra of the cleaved Suzuki coupling products}

Figure S21. ${ }^{1} \mathrm{H}$ and ${ }^{13} \mathrm{C} \mathrm{NMR}$ spectra of 5a
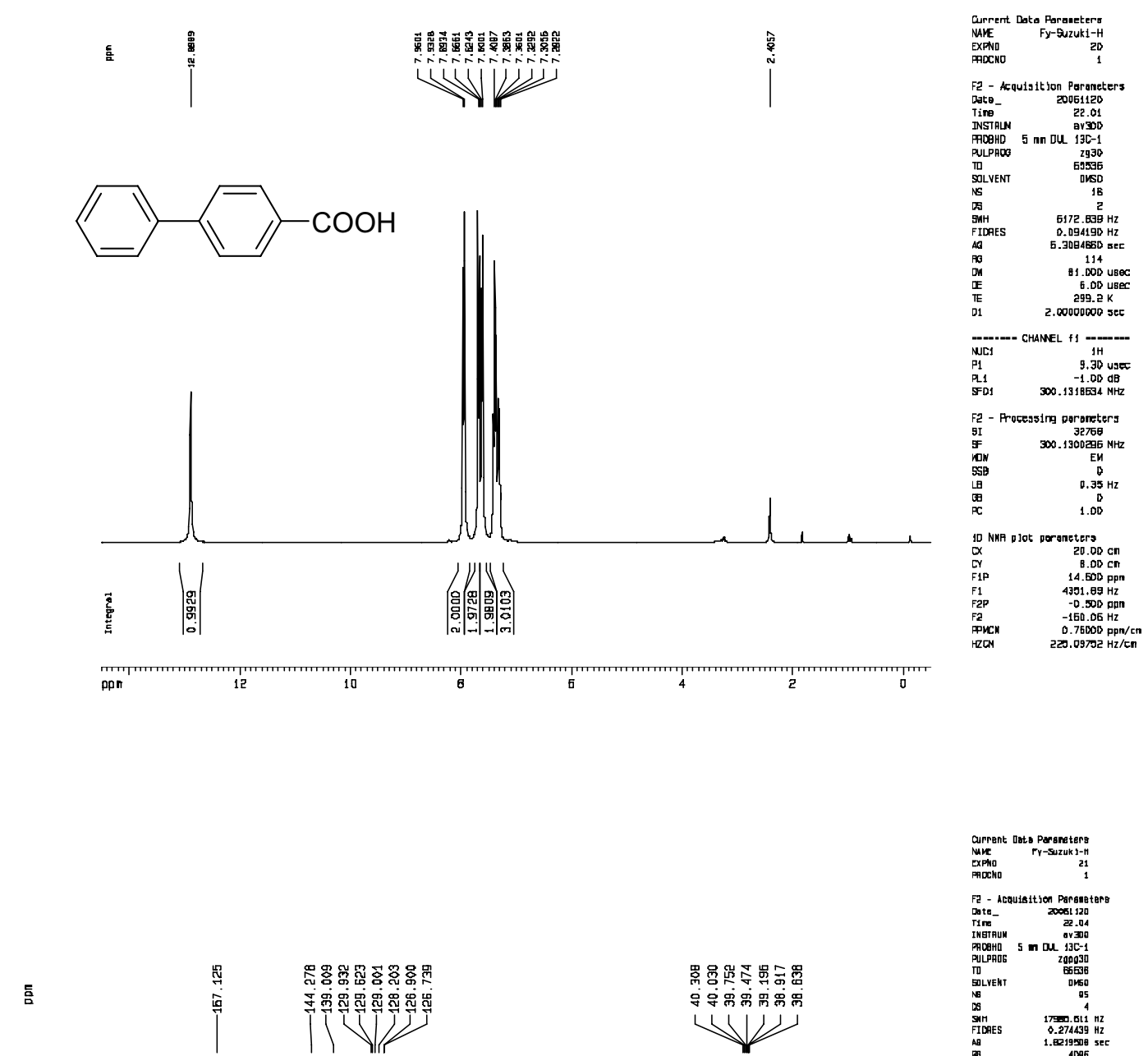

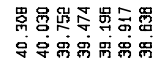
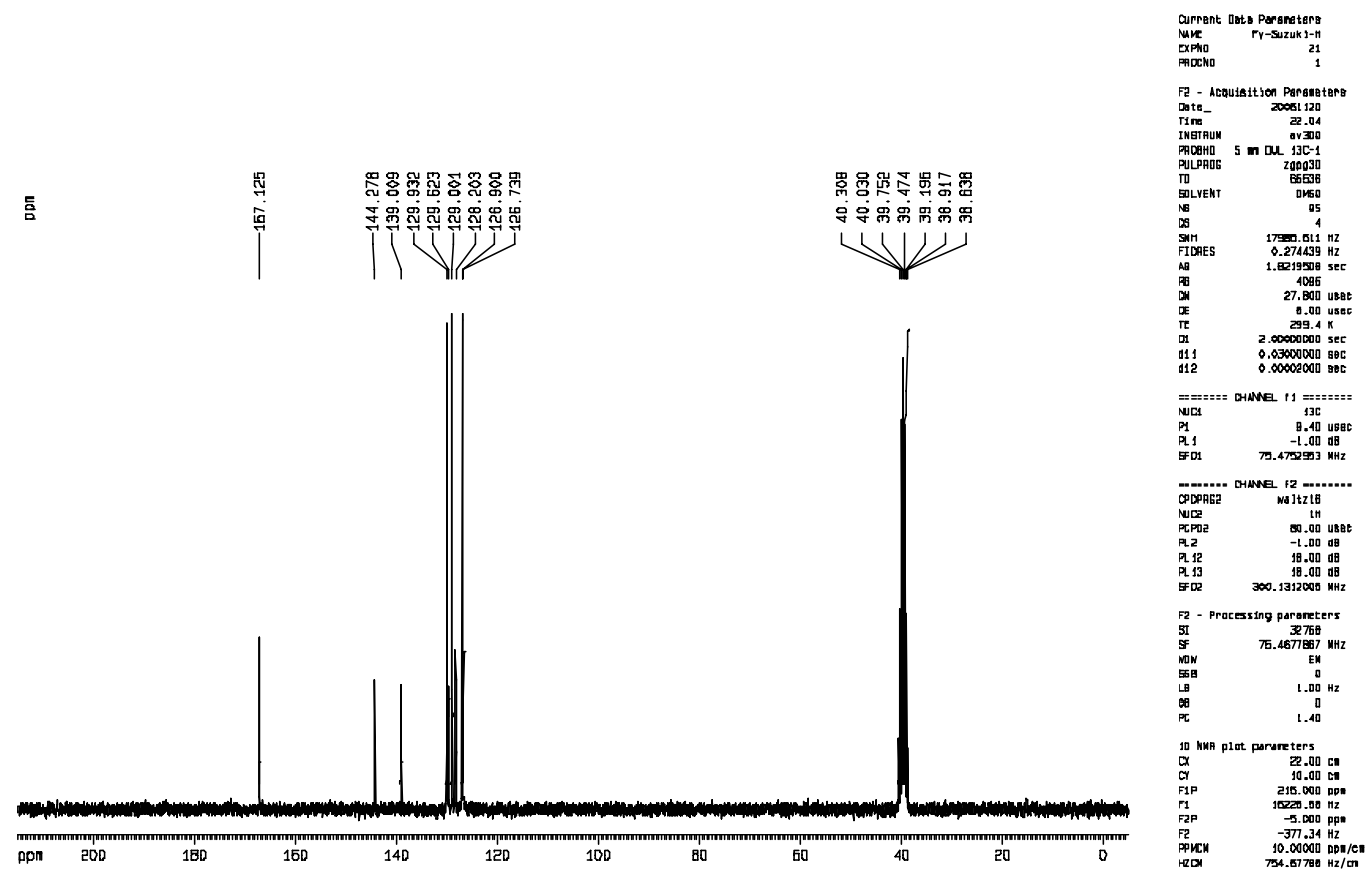
Figure S22. ${ }^{1} \mathrm{H}$ and ${ }^{13} \mathrm{C}$ NMR spectra of $\mathbf{5 b}$

Fy-Suzuki-CH3
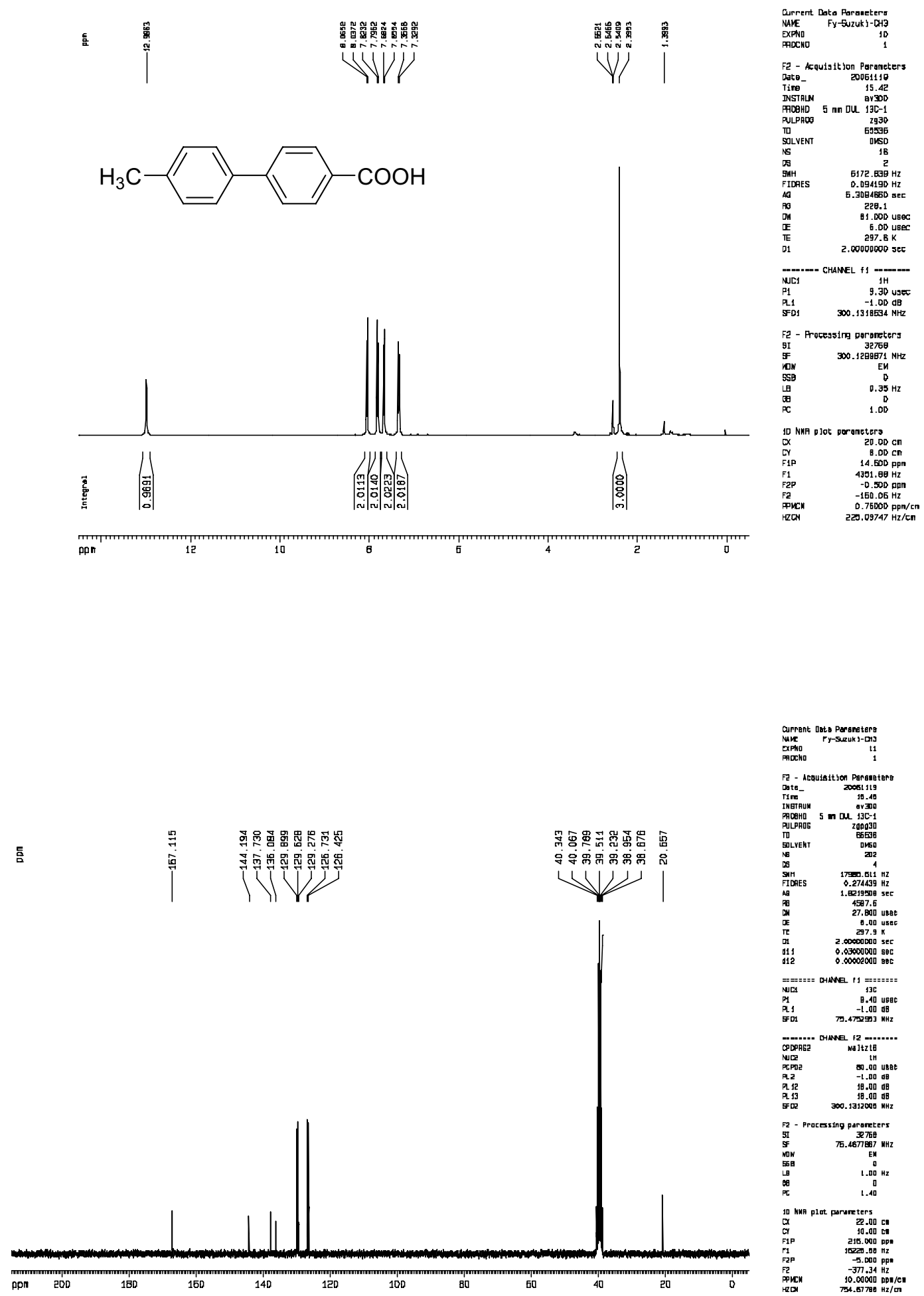
Figure S23. ${ }^{1} \mathrm{H}$ and ${ }^{13} \mathrm{C}$ NMR spectra of 5c
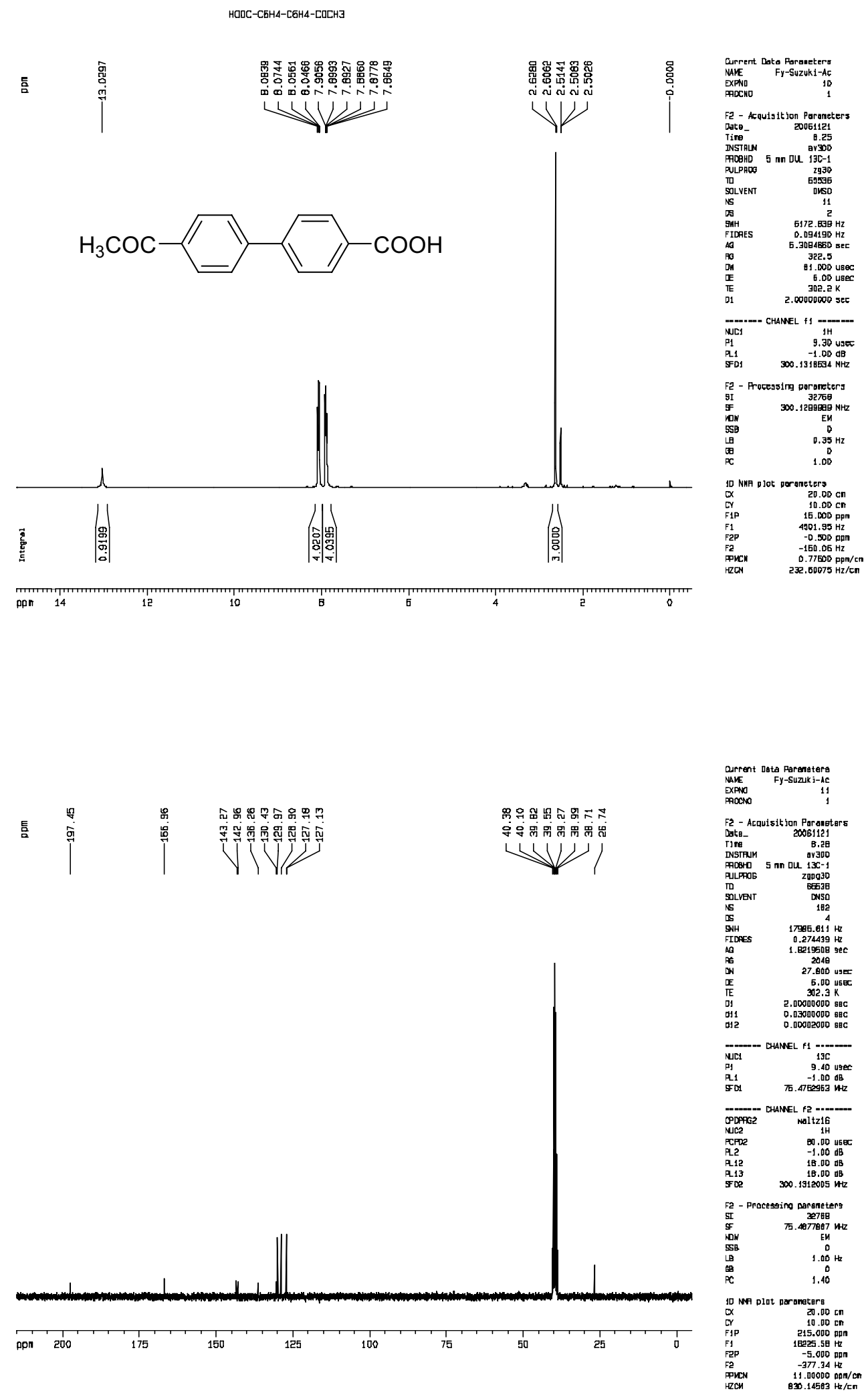
Figure S24. ${ }^{1} \mathrm{H}$ and ${ }^{13} \mathrm{C}$ NMR spectra of $\mathbf{5 d}$
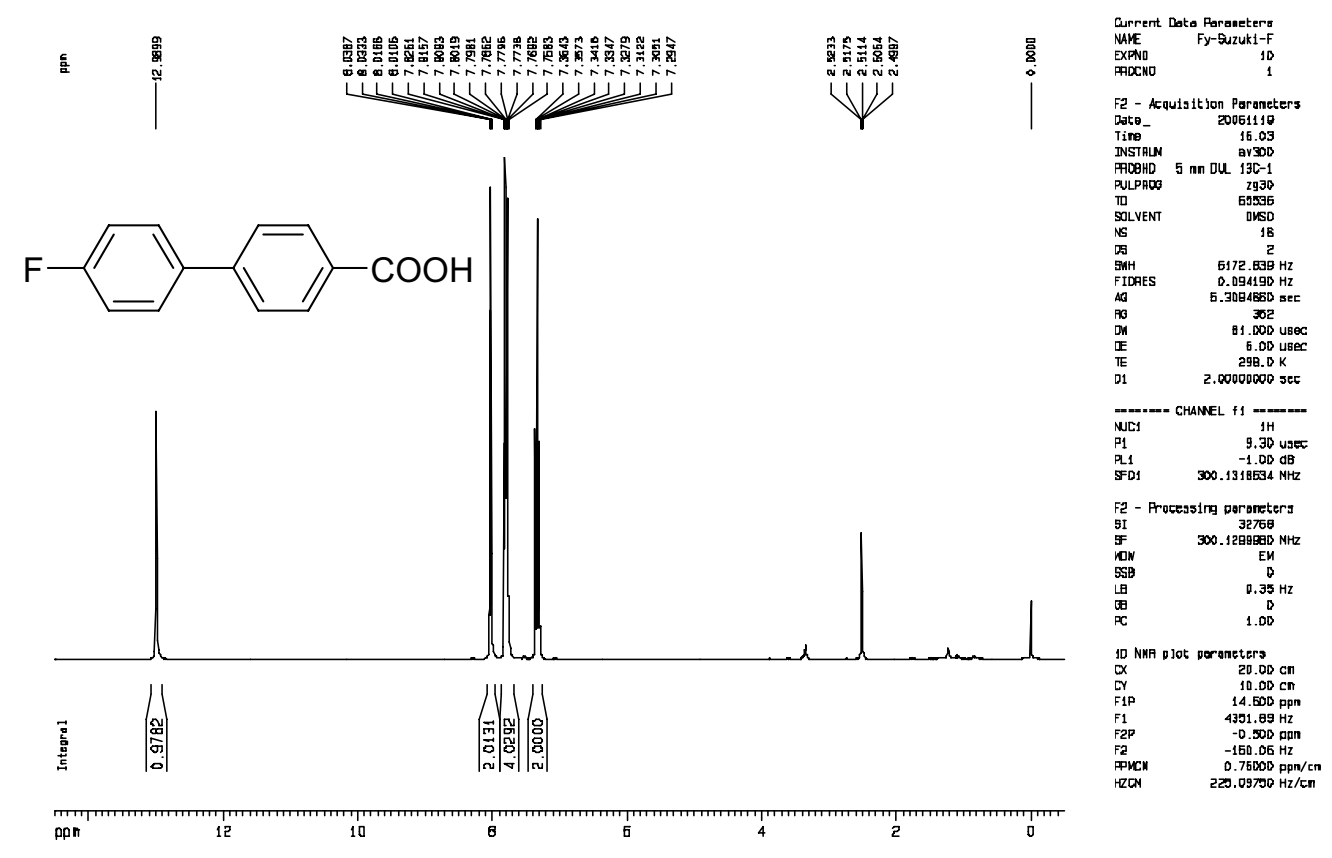

Fy-Suzuki-F

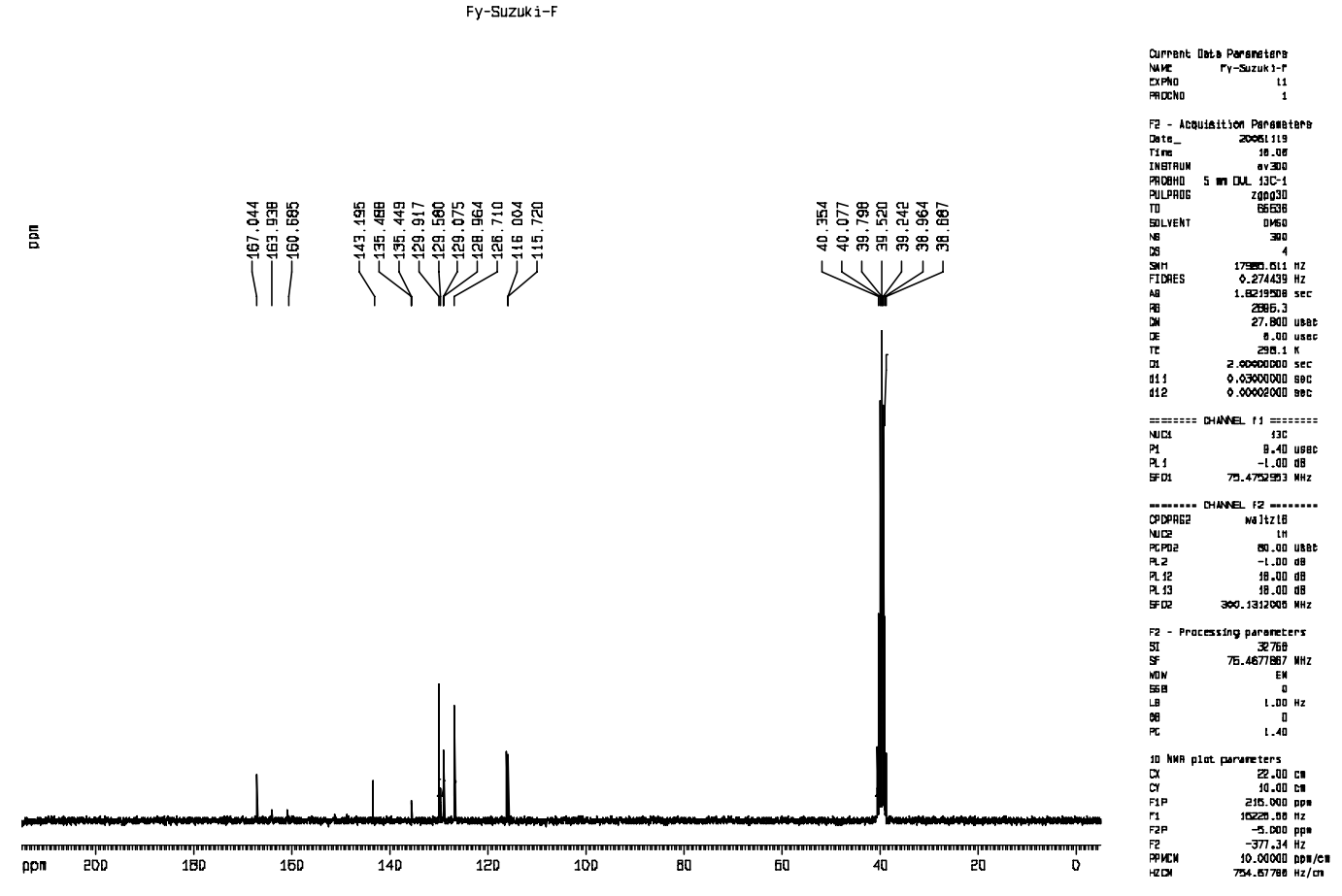




\section{7. ${ }^{1} \mathrm{H}$ NMR spectra of $\mathrm{G}_{3} \mathrm{G}_{2}$-COOMe.}

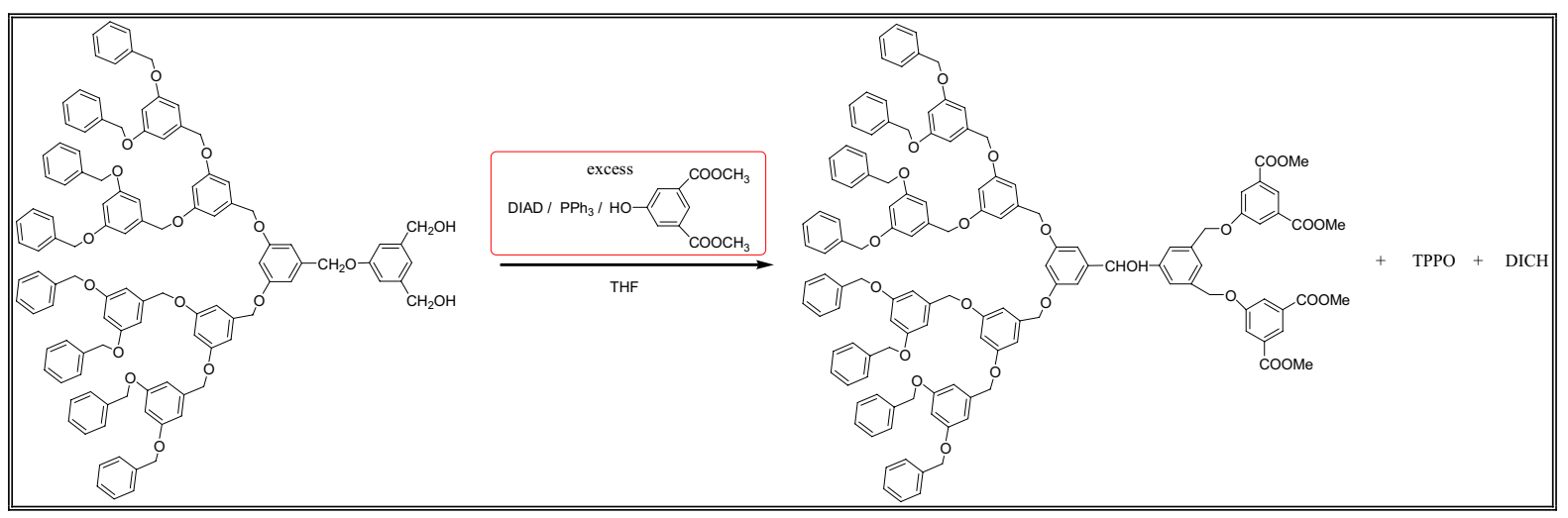

a) Before work-up

Janus dendriner G36'2COaMe - chuliqian
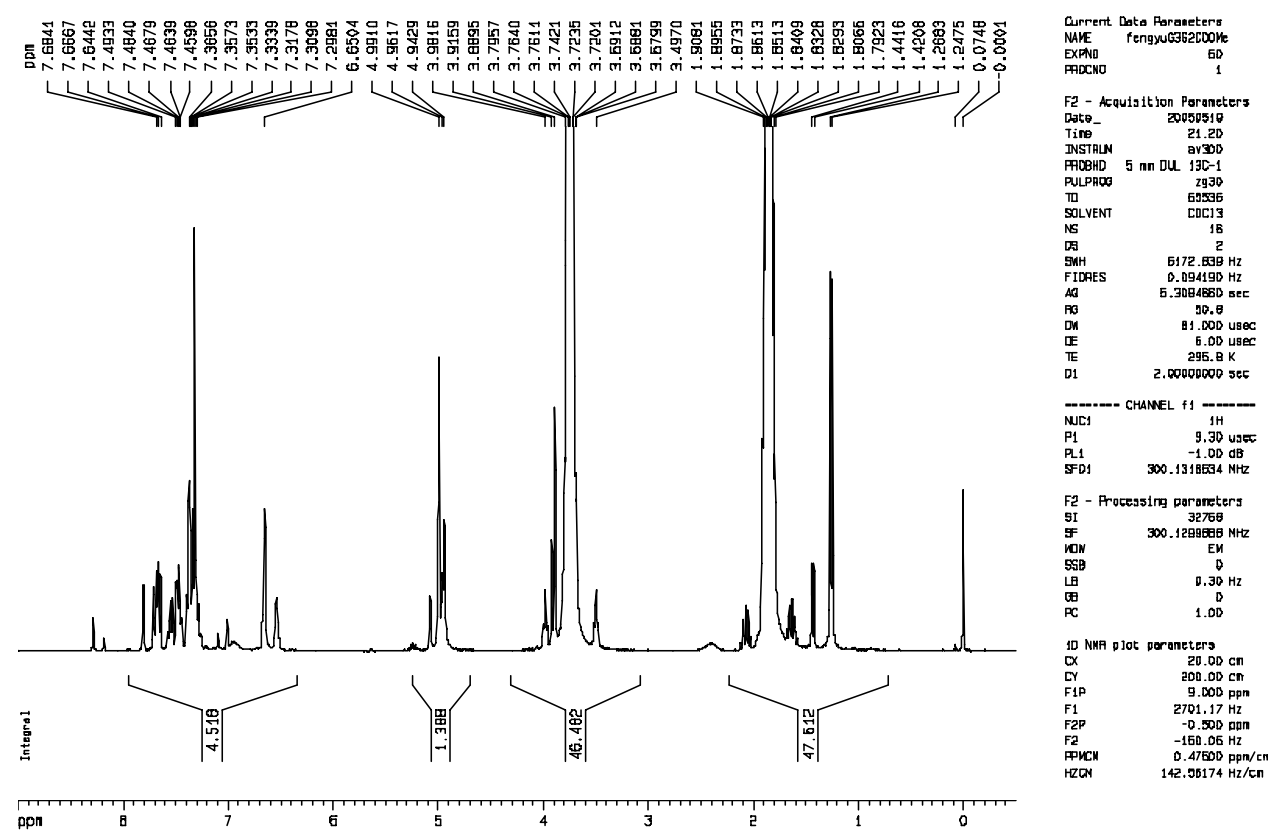
b) After precipitation by methanol and diethyl ether

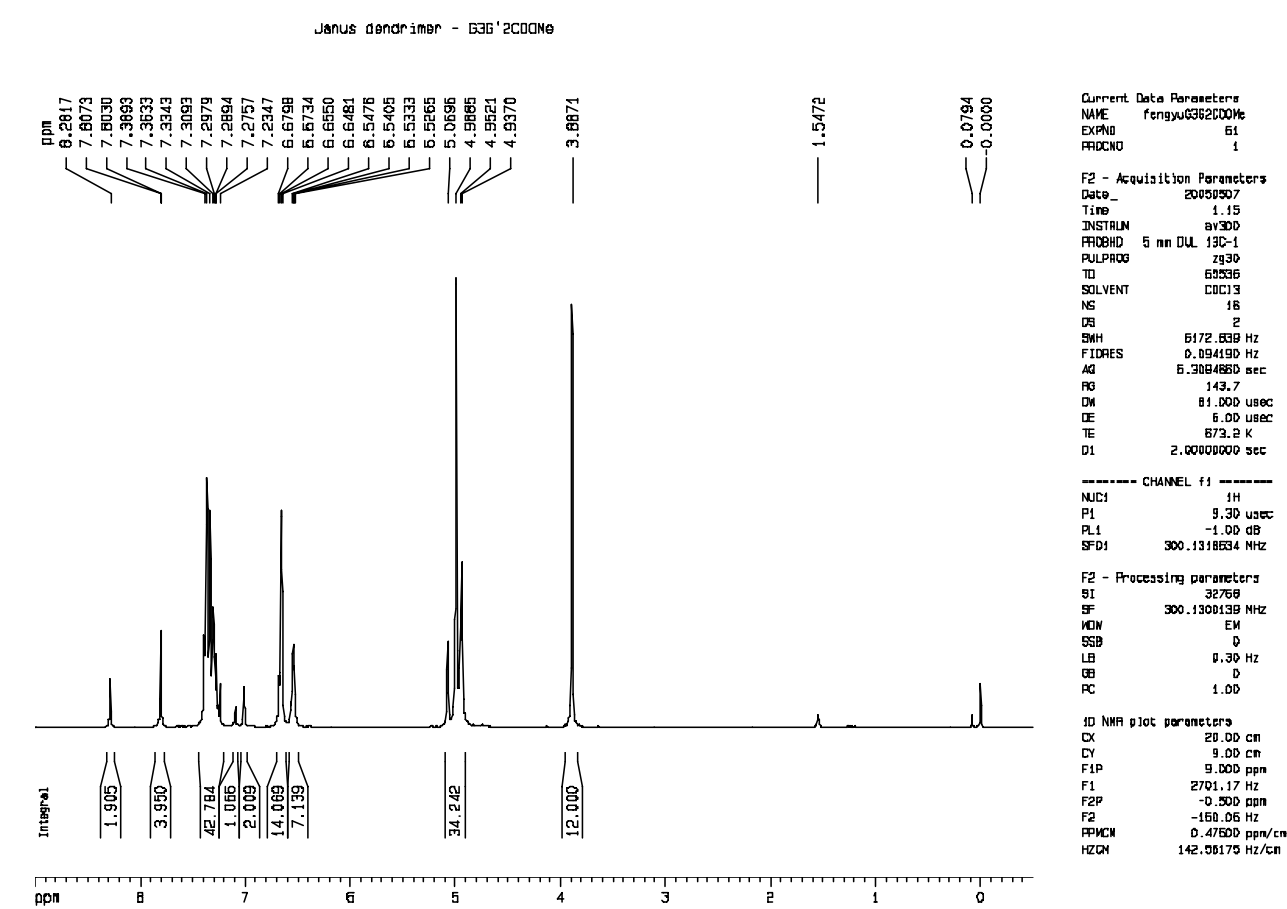

\section{8. ${ }^{1} \mathrm{H}$ NMR spectra of $4 \mathrm{ca}$.}

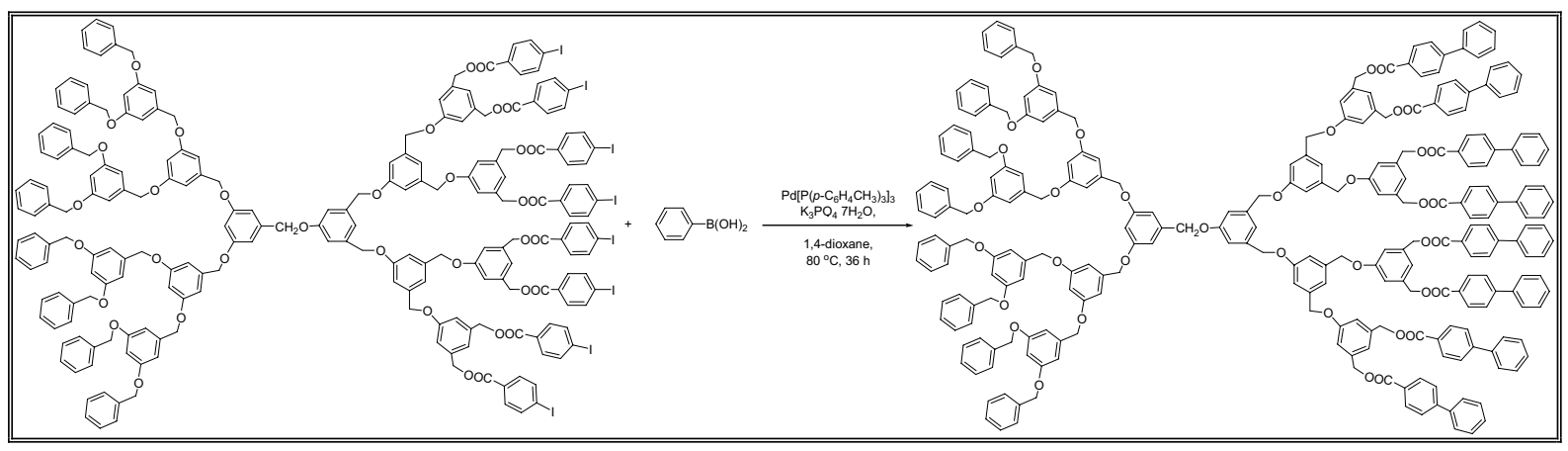


a). Before work-up

Fy-G3G'3-5uzUki-UP
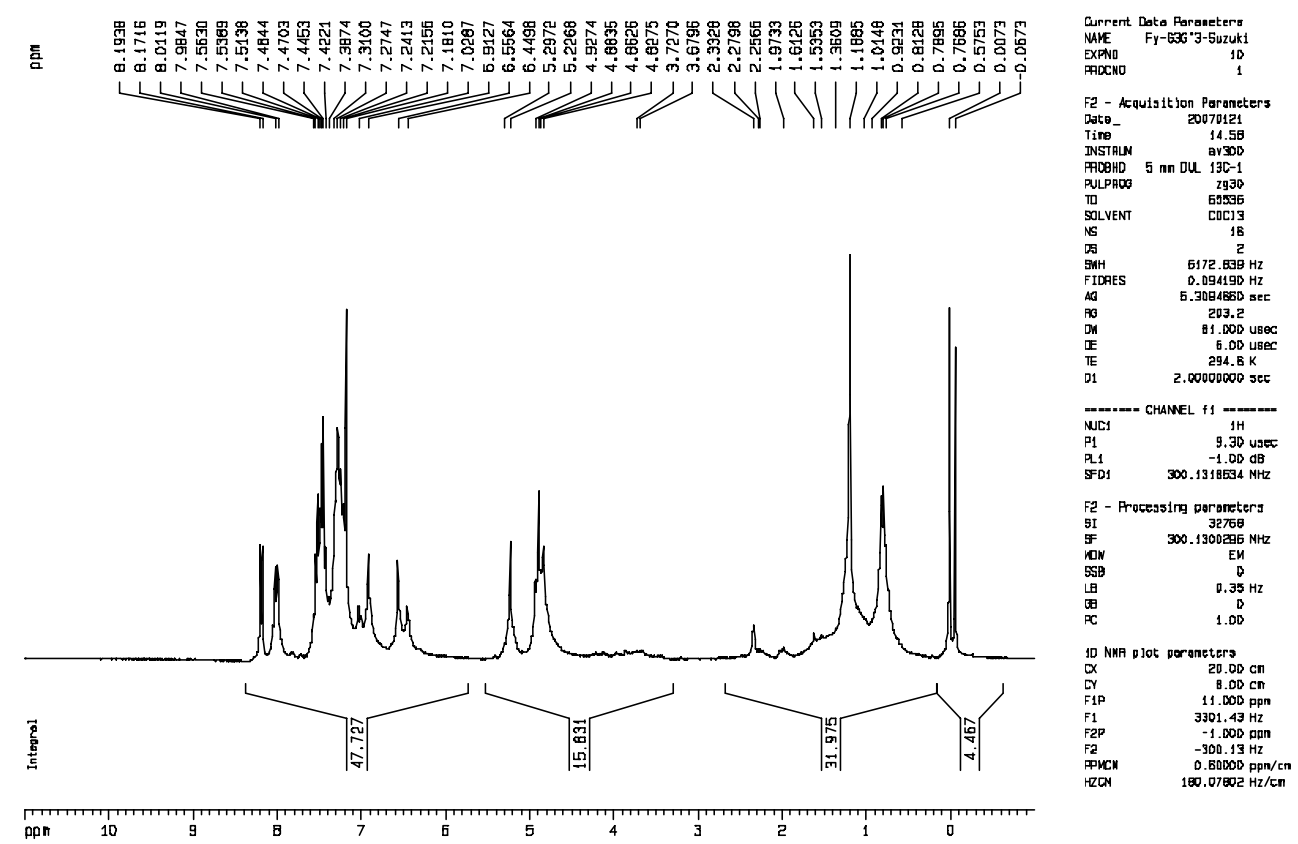

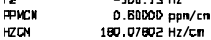

b). After twice precipitation by methanol

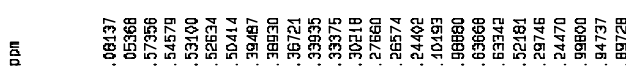
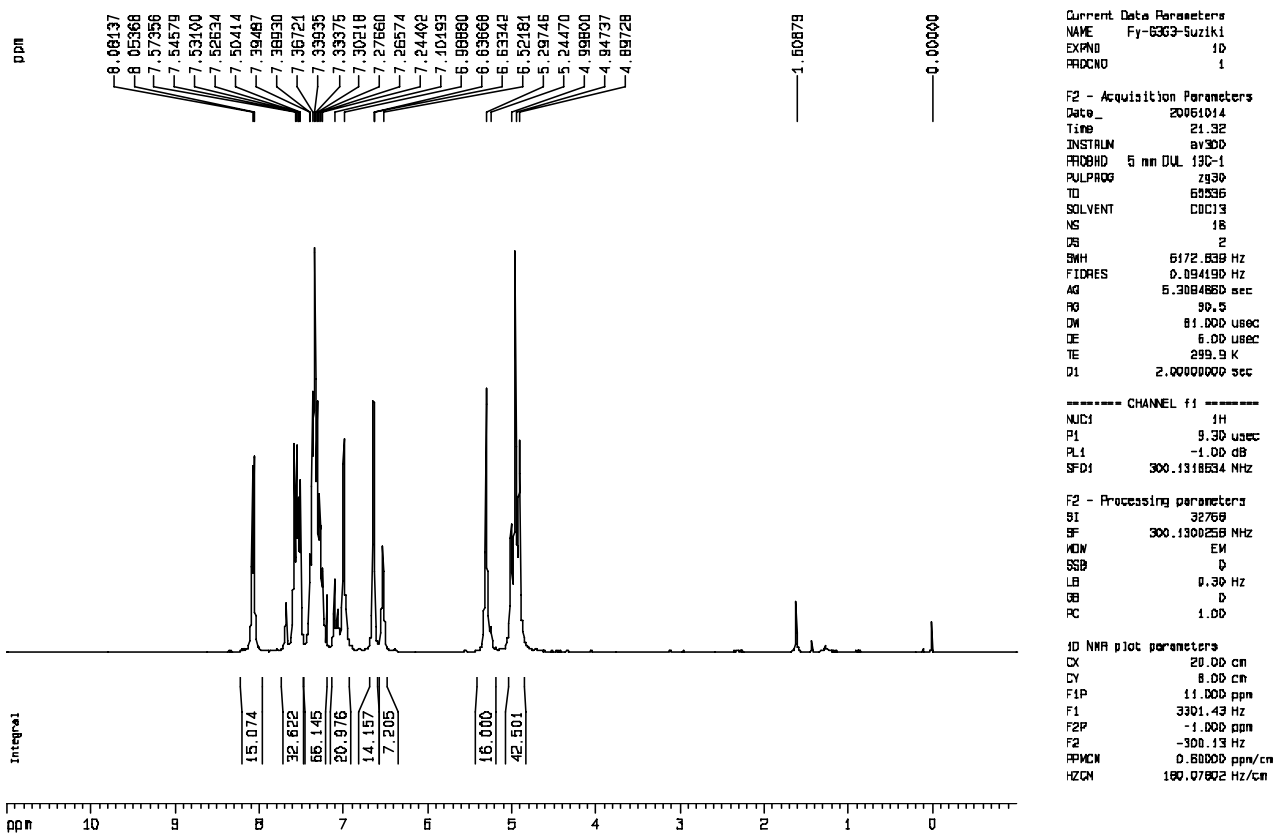


\section{References}

1. Hawker, C. J.; Fréchet, J. M. J. J. Am. Chem. Soc. 1990, 112, 7638-7647.

2. Tolman, C. A.; Seidel, W. C.; Gerlach, D. H.; J. Am. Chem. Soc. 1972; 94, 2669-2676. 